Supporting Information

\title{
Copper-Catalyzed Construction of Benzo[4,5]imidazo[2,1-a] isoquinolines Using Calcium Carbide as a Solid Alkyne Source
}

\author{
Haiyan Liu, Zheng Li* \\ College of Chemistry and Chemical Engineering, Northwest Normal University, \\ Lanzhou, Gansu, 730070, P. R. China
}

\section{Table of contents}

1. Condition optimization (page S2)

2. Experimental section (page S4)

2.1 General information

2.2 The general procedure for the synthesis of benzo[4,5]imidazo[2,1-a]isoquinolines $\mathbf{2 a - 2 u}$

2.3 Gram-scale synthesis of benzo[4,5]imidazo[2,1-a]isoquinoline (2a)

2.4 One-pot three-component synthesis of benzo[4,5]imidazo[2,1-a]isoquinoline (2a)

2.5 Gram-scale synthesis of benzo[4,5]imidazo[2,1-a]isoquinoline (2a) through one-pot three-component procedure

3. Analytical data for compounds $\mathbf{2 a - 2 u}$ (page S6)

4. ${ }^{1} \mathrm{H},{ }^{13} \mathrm{C}$ and ${ }^{19} \mathrm{~F}$ NMR spectra for products $\mathbf{2 a - 2 u}$ (page S15)

References (page S39) 


\section{Condition optimization}

Table S1 The effect of the amount of catalyst on the yield of $\mathbf{2} \mathbf{a}^{[a]}$

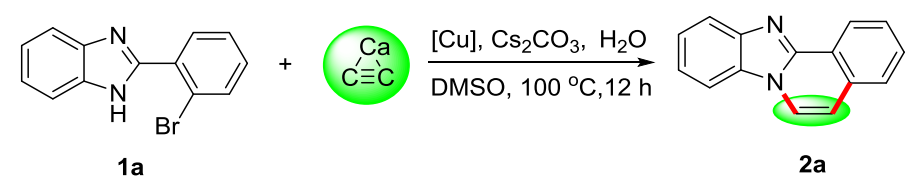

\begin{tabular}{llllcc}
\hline Entry & $\begin{array}{l}\text { Catalyst } \\
\text { (amount) }\end{array}$ & Base & Solvent & Temp $\left({ }^{\circ} \mathrm{C}\right)$ & Yield $(\%)^{[\mathrm{b}]}$ \\
\hline 1 & $\mathrm{CuI}(0)$ & $\mathrm{Cs}_{2} \mathrm{CO}_{3}$ & DMSO & 100 & 0 \\
2 & $\mathrm{CuI}(1 \%)$ & $\mathrm{Cs}_{2} \mathrm{CO}_{3}$ & DMSO & 100 & 12 \\
3 & $\mathrm{CuI}(2 \%)$ & $\mathrm{Cs}_{2} \mathrm{CO}_{3}$ & DMSO & 100 & 25 \\
4 & $\mathrm{CuI}(5 \%)$ & $\mathrm{Cs}_{2} \mathrm{CO}_{3}$ & DMSO & 100 & 50 \\
$\mathbf{5}$ & $\mathrm{CuI} \mathrm{(10 \% )}$ & $\mathrm{Cs}_{2} \mathrm{CO}_{3}$ & DMSO & $\mathbf{1 0 0}$ & $\mathbf{8 2}$ \\
6 & $\mathrm{CuI}(20 \%)$ & $\mathrm{Cs}_{2} \mathrm{CO}_{3}$ & DMSO & 100 & 82 \\
\hline
\end{tabular}

${ }^{[a]}$ Reaction condition: 1a $(0.5 \mathrm{mmol})$, calcium carbide $(1.25 \mathrm{mmol}), \mathrm{H}_{2} \mathrm{O}(1.5 \mathrm{mmol})$, $\mathrm{Cs}_{2} \mathrm{CO}_{3}(0.5 \mathrm{mmol}), \mathrm{CuI}$ (appropriate amount) in $4 \mathrm{~mL}$ of DMSO stirred at $100{ }^{\circ} \mathrm{C}$ for $12 \mathrm{~h}$ under nitrogen atmosphere. ${ }^{[\mathrm{b}]}$ Isolated yield.

Table S2 The effect of the amount of calcium carbide on the yield of $\mathbf{2 a}^{[\mathrm{a}]}$

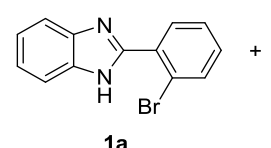

$\frac{\mathrm{Cul}, \mathrm{Cs}_{2} \mathrm{CO}_{3}, \mathrm{H}_{2} \mathrm{O}}{\mathrm{DMSO}, 100^{\circ} \mathrm{C}, 12 \mathrm{~h}}$

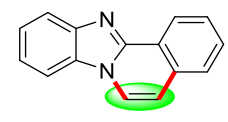

1a

\begin{tabular}{clllcc}
\hline \multirow{2}{*}{ Entry } & $\mathrm{CaC}_{2}(\mathrm{mmol})$ & Base & Solvent & $\mathrm{Temp}\left({ }^{\circ} \mathrm{C}\right)$ & Yield $(\%)^{[\mathrm{b}]}$ \\
\hline 1 & $\mathrm{CaC}_{2}(0.5)$ & $\mathrm{Cs}_{2} \mathrm{CO}_{3}$ & DMSO & 100 & 55 \\
2 & $\mathrm{CaC}_{2}(1)$ & $\mathrm{Cs}_{2} \mathrm{CO}_{3}$ & DMSO & 100 & 70 \\
$\mathbf{3}$ & $\mathrm{CaC}_{2}(\mathbf{1 . 2 5})$ & $\mathrm{Cs}_{2} \mathrm{CO}_{3}$ & DMSO & $\mathbf{1 0 0}$ & $\mathbf{8 2}$ \\
4 & $\mathrm{CaC}_{2}(1.5)$ & $\mathrm{Cs}_{2} \mathrm{CO}_{3}$ & DMSO & 100 & 82 \\
5 & $\mathrm{CaC}_{2}(2)$ & $\mathrm{Cs}_{2} \mathrm{CO}_{3}$ & DMSO & 100 & 80 \\
\hline
\end{tabular}


${ }^{\text {[a] }}$ Reaction condition: 1a $(0.5 \mathrm{mmol})$, calcium carbide (appropriate amount), $\mathrm{H}_{2} \mathrm{O}$ (1.5 $\mathrm{mmol}), \mathrm{Cs}_{2} \mathrm{CO}_{3}(0.5 \mathrm{mmol}), \mathrm{CuI}(0.05 \mathrm{mmol})$ in $4 \mathrm{~mL}$ of DMSO stirred at $100{ }^{\circ} \mathrm{C}$ for $12 \mathrm{~h}$ under nitrogen atmosphere. ${ }^{[\mathrm{b}]}$ Isolated yield.

\section{HRMS for intermediate $\mathrm{E}$}

HRMS (ESI): $m / z(\mathrm{M}-\mathrm{Cu}+\mathrm{H})^{+}$calcd for $\mathrm{C}_{15} \mathrm{H}_{11} \mathrm{~N}_{2:}$ 219.0917; Found: 219.0917.

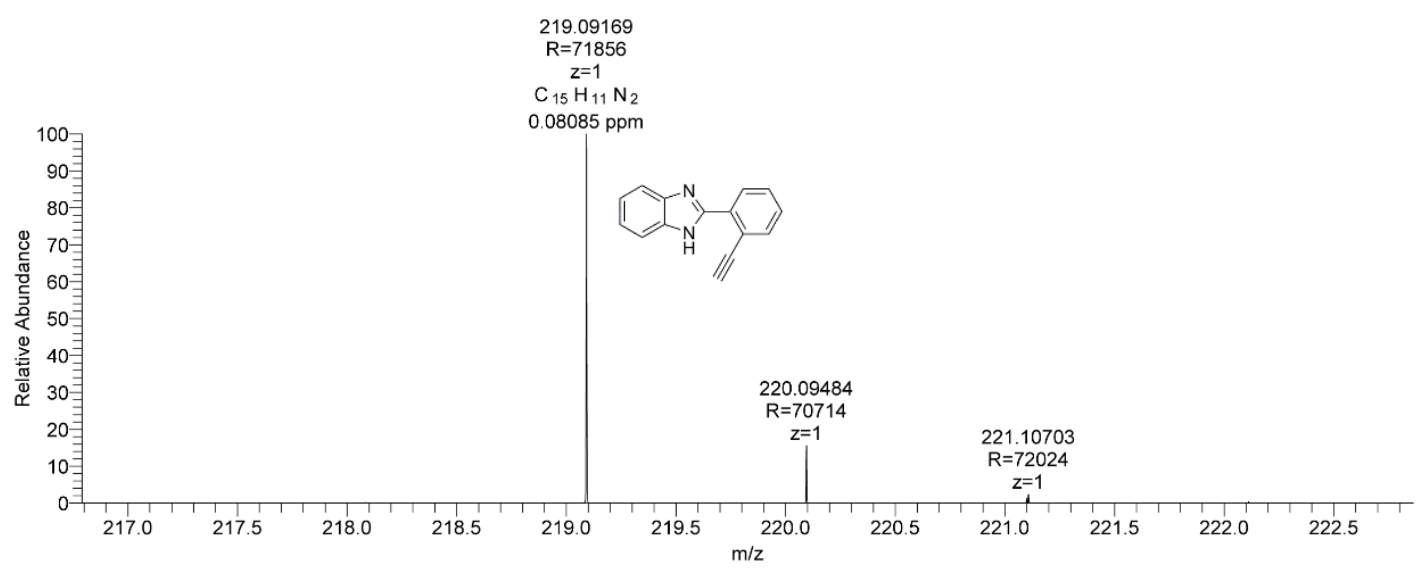

Figure S1 HRMS spectra for intermediate $\mathbf{E}$ 


\section{Experimental Section}

\subsection{General Information}

${ }^{1} \mathrm{H}$ NMR and ${ }^{13} \mathrm{C}$ NMR spectra were recorded on a Mercury-600 MB or $400 \mathrm{MB}$ instrument using $\mathrm{CDCl}_{3}$ as solvent and $\mathrm{Me}_{4} \mathrm{Si}$ as internal standard. High-resolution mass spectra (HRMS) (ESI) were obtained with a Bruker Daltonics APEX II 47e and quadrupole Orbitrap Elite (Q-Exactive) mass spectrometer. Melting points were observed in an electrothermal melting point apparatus (X-5, Beijing Tech Instrument Co. Ltd, China). Calcium carbide was purchased from Macklin Chemical Company (China, purity: 98\%), and ground into powder (ca. 50-100 mesh) in a ceramic mortar prior to use. The solvents were all deoxygenated with nitrogen gas for $5 \mathrm{~min}$ and dehydrated with molecular sieve for over $12 \mathrm{~h}$ prior to use. Unless otherwise noted, the reagents were reagent grade and used without any further purification. Column chromatography was carried out on a flash chromatographic system using silica gel, and petroleum ether $\left(60-90{ }^{\circ} \mathrm{C}\right)$ and ethyl acetate as eluent. For thin layer chromatography (TLC), silica gel plates precoated with GF-254 were used. Various benzimidazoles were synthesized by the reactions of corresponding $o$-phenylenediamines and $o$-bromobenzaldehydes according to literature procedure. ${ }^{[1]}$

2.2 The general procedure for the synthesis of benzo[4,5]imidazo[2,1-a]isoquinolines $2 \mathrm{a}-2 \mathrm{u}$

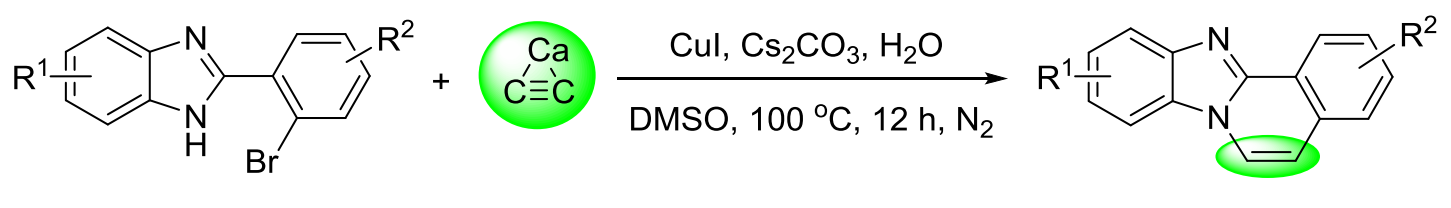

2-(2-Bromophenyl)-1H-benzo[d]imidazoles $1(0.5 \mathrm{mmol})$, calcium carbide $(1.25$ mmol), cuprous iodide $(0.05 \mathrm{mmol})$, cesium carbonate $(0.5 \mathrm{mmol})$ and water $(1.5$ mmol) in dimethyl sulfoxide ( $4 \mathrm{~mL})$ were heated in an oil bath to $100{ }^{\circ} \mathrm{C}$ for $12 \mathrm{~h}$ under nitrogen atmosphere. After the completion of the reaction, the resulting mixture was filtered to remove the solid, and the liquor was extracted with ethyl acetate $(3 \times 10$ $\mathrm{mL})$, and washed with saturated brine $(3 \times 10 \mathrm{~mL})$. The resulting organic phase was dried with anhydrous sodium sulfate, and concentrated under reduced pressure. The residue was isolated by column chromatography using petroleum ether and ethyl acetate (v/v 8:1) as eluent to give the pure products 2 . 
2.3 Gram-scale synthesis of benzo[4,5]imidazo[2,1-a]isoquinoline (2a)

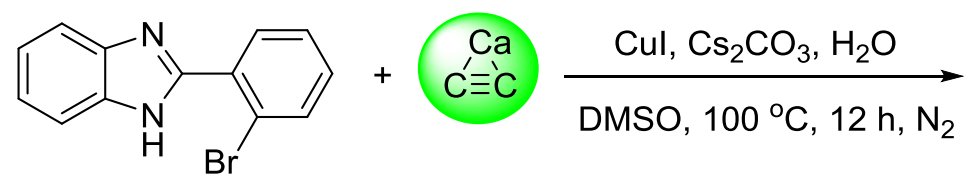

$1 \mathrm{a} \quad 4.09 \mathrm{~g}$

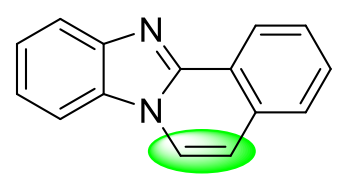

2a $\quad 2.36 \mathrm{~g}, 72 \%$

2-(2-Bromophenyl)-1H-benzo[ $d]$ imidazole (1a) (4.09 g, $15 \mathrm{mmol})$, calcium carbide (2.45 g, $37.5 \mathrm{mmol})$, cuprous iodide $(0.28 \mathrm{~g}, 1.5 \mathrm{mmol})$, cesium carbonate $(4.88 \mathrm{~g}$, $15 \mathrm{mmol})$ and water $(0.81 \mathrm{~mL}, 45 \mathrm{mmol})$ in dimethyl sulfoxide $(60 \mathrm{~mL})$ were heated in an oil bath to $100{ }^{\circ} \mathrm{C}$ for $12 \mathrm{~h}$ under nitrogen atmosphere. After the completion of the reaction, the resulting mixture was filtered to remove the solid, and the liquor was extracted with ethyl acetate $(3 \times 50 \mathrm{~mL})$, and washed with saturated brine $(3 \times 50 \mathrm{~mL})$. The resulting organic phase was dried with anhydrous sodium sulfate, and concentrated under reduced pressure. The residue was isolated by column chromatography using petroleum ether and ethyl acetate (v/v 8:1) as eluent to give the pure product $\mathbf{2 a}(2.36 \mathrm{~g}, 72 \%)$.

2.4 One-pot three-component synthesis of benzo[4,5]imidazo[2,1-a]isoquinoline (2a)<smiles>Nc1ccccc1N</smiles>

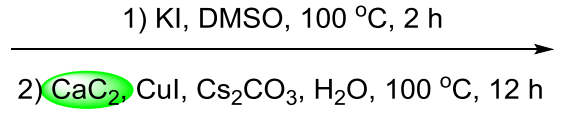

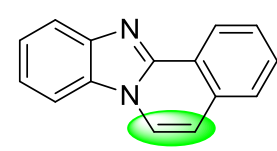

2a $71 \%$

$o$-Phenylenediamine (54 mg, $0.5 \mathrm{mmol}$ ), $o$-bromobenzaldehyde (102 $\mathrm{mg}, 0.55$ $\mathrm{mmol}$ ) and potassium iodide $(83 \mathrm{mg}, 0.5 \mathrm{mmol})$ in DMSO $(5 \mathrm{~mL})$ were heated in an oil bath to $100{ }^{\circ} \mathrm{C}$ in an open flask for $2 \mathrm{~h}$, and the reaction progress was monitored by TLC. After the $o$-phenylenediamine was completely consumed, calcium carbide (82 $\mathrm{mg}, 1.25 \mathrm{mmol})$, cuprous iodide $(9.5 \mathrm{mg}, 0.05 \mathrm{mmol})$, cesium carbonate (163 mg, 0.5 $\mathrm{mmol})$, and water $(18 \mathrm{mg}, 1 \mathrm{mmol})$ were added to the reaction system, and the mixture was continued to heat in an oil bath to $100{ }^{\circ} \mathrm{C}$ for $12 \mathrm{~h}$. After the completion of the reaction, the solid was removed by filtration, and the liquor was extracted with ethyl acetate $(3 \times 10 \mathrm{~mL})$, and washed with saturated brine $(3 \times 10 \mathrm{~mL})$. The resulting organic phase was dried with anhydrous sodium sulfate, and concentrated under reduced pressure. The residue was isolated by column chromatography using petroleum ether and ethyl acetate as eluent (v/v 8:1) to give the pure product. 


\subsection{Gram-scale synthesis of benzo[4,5]imidazo[2,1-a]isoquinoline (2a) through}

one-pot three-component procedure<smiles>Nc1ccccc1N</smiles>

$0.87 \mathrm{~g}$<smiles>O=Cc1ccccc1Br</smiles>

$1.63 \mathrm{~g}$

1) $\mathrm{KI}, \mathrm{DMSO}, 100^{\circ} \mathrm{C}, 2 \mathrm{~h}$

2) $\mathrm{CaC}_{2}, \mathrm{Cul}, \mathrm{Cs}_{2} \mathrm{CO}_{3}, \mathrm{H}_{2} \mathrm{O}, 100^{\circ} \mathrm{C}, 12 \mathrm{~h}$

$o$-Phenylenediamine $(0.87 \mathrm{~g}, 8 \mathrm{mmol}), o$-bromobenzaldehyde $(1.63 \mathrm{~g}, 8.8 \mathrm{mmol})$ and potassium iodide $(1.33 \mathrm{~g}, 8 \mathrm{mmol})$ in DMSO $(50 \mathrm{~mL})$ were heated in an oil bath to $100{ }^{\circ} \mathrm{C}$ in an open flask for $2 \mathrm{~h}$, and the reaction progress was monitored by TLC. After the $o$-phenylenediamine was completely consumed, calcium carbide $(1.31 \mathrm{~g}, 20$ mmol), cuprous iodide $(0.15 \mathrm{~g}, 0.8 \mathrm{mmol})$, cesium carbonate $(2.61 \mathrm{~g}, 8 \mathrm{mmol})$, and water $(0.29 \mathrm{~g}, 16 \mathrm{mmol})$ were added to the reaction system, and the mixture was continued to heat in an oil bath to $100{ }^{\circ} \mathrm{C}$ for $12 \mathrm{~h}$. After the completion of the reaction, the solid was removed by filtration, and the liquor was extracted with ethyl acetate $(3 \times 30 \mathrm{~mL})$, and washed with saturated brine $(3 \times 30 \mathrm{~mL})$. The resulting organic phase was dried with anhydrous sodium sulfate, and concentrated under reduced pressure. The residue was isolated by column chromatography using petroleum ether and ethyl acetate as eluent (v/v 8:1) to give the pure product $\mathbf{2 a}(1.17 \mathrm{~g}, 67 \%)$.

\section{Analytical data for compounds $2 a-2 u$}

\subsection{Benzo[4,5]imidazo[2,1-a]isoquinoline $(2 a)^{[2]}$}<smiles>c1ccc2c(c1)ccn1c3ccccc3nc21</smiles>

Yellow solid (89.4 mg, 82\% yield). M.p. $119-121{ }^{\circ} \mathrm{C} ;{ }^{1} \mathrm{H}$ NMR: (400 MHz, $\mathrm{CDCl}_{3}$ ): $\delta 8.78(\mathrm{~d}, J=7.8 \mathrm{~Hz}, 1 \mathrm{H}), 8.01(\mathrm{t}, J=7.1 \mathrm{~Hz}, 2 \mathrm{H}), 7.72(\mathrm{~d}, J=8.1 \mathrm{~Hz}, 1 \mathrm{H}), 7.66-$ $7.59(\mathrm{~m}, 3 \mathrm{H}), 7.48$ (t, $J=7.7 \mathrm{~Hz}, 1 \mathrm{H}), 7.35$ (t, $J=7.6 \mathrm{~Hz}, 1 \mathrm{H}), 6.94(\mathrm{~d}, J=7.3 \mathrm{~Hz}, 1 \mathrm{H})$ ppm; ${ }^{13} \mathrm{C}$ NMR $\left(150 \mathrm{MHz}, \mathrm{CDCl}_{3}\right) \delta 147.1,143.7,131.5,130.0,129.9,128.1,128.0$, $124.9,124.6,123.5,121.8,121.2,119.8,111.2,109.7$ ppm.

3.2 10-Methylbenzo[4,5]imidazo[2,1-a]isoquinoline and 9-methylbenzo [4,5]imidazo[2,1-a]isoquinoline $(2 \boldsymbol{b})^{[2]}$ 


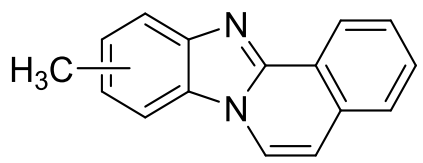

Yellow solid (80.1 mg, 69\% yield). M.p. $156-163{ }^{\circ} \mathrm{C} ;{ }^{1} \mathrm{H}$ NMR (400 MHz, $\left.\mathrm{CDCl}_{3}\right) \delta$ $8.82-8.79(\mathrm{~m}, 1 \mathrm{H}), 8.12(\mathrm{t}, J=7.9 \mathrm{~Hz}, 1 \mathrm{H}), 7.88(\mathrm{~d}, J=8.3 \mathrm{~Hz}, 0.66 \mathrm{H}), 7.78(\mathrm{~d}, J=$ $3.4 \mathrm{~Hz}, 0.34 \mathrm{H}), 7.74-7.62(\mathrm{~m}, 4 \mathrm{H}), 7.33(\mathrm{~d}, J=8.4 \mathrm{~Hz}, 0.66 \mathrm{H}), 7.23(\mathrm{~d}, J=8.9 \mathrm{~Hz}$, $0.34 \mathrm{H}), 7.05(\mathrm{t}, J=6.1,1 \mathrm{H}), 2.60(\mathrm{~s}, 1.98 \mathrm{H}), 2.58(\mathrm{~s}, 1.02 \mathrm{H}) \mathrm{ppm} ;{ }^{13} \mathrm{C} \mathrm{NMR}(150$ $\left.\mathrm{MHz}, \mathrm{CDCl}_{3}\right) \delta 146.9,141.2,134.6,132.0,131.5,131.4,130.2,129.8,129.7,128.2$, 128.1, 127.0, 126.9, 126.4, 124.9, 124.8, 123.7, 123.6, 123.5, 121.4, 121.3, 119.5, 119.3, 111.2, 111.1, 109.6, 109.2, 22.0, 21.9 ppm.

3.3 10-Methoxybenzo[4,5]imidazo[2,1-a]isoquinoline and 9-methoxybenzo [4,5]imidazo[2,1-a]isoquinoline $(2 \mathrm{c})^{[2]}$

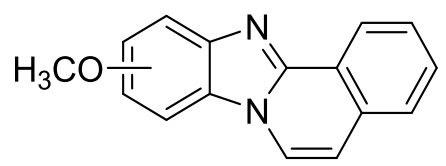

Yellow solid (105.4 mg, 85\% yield). M.p. $125-134{ }^{\circ} \mathrm{C} ;{ }^{1} \mathrm{H}$ NMR (400 MHz, $\mathrm{CDCl}_{3}$ ) $\delta 8.79-8.73(\mathrm{~m}, 1 \mathrm{H}), 8.07(\mathrm{~d}, J=7.2 \mathrm{~Hz}, 0.7 \mathrm{H}), 8.01(\mathrm{~d}, J=7.3 \mathrm{~Hz}, 0.3 \mathrm{H}), 7.88(\mathrm{~d}, J$ $=9.1 \mathrm{~Hz}, 0.3 \mathrm{H}), 7.73-7.60(\mathrm{~m}, 3.7 \mathrm{H}), 7.45(\mathrm{~d}, J=2.3 \mathrm{~Hz}, 0.7 \mathrm{H}), 7.21(\mathrm{~d}, J=2.3 \mathrm{~Hz}$, $0.3 \mathrm{H}), 7.14(\mathrm{dd}, J=8.9,2.4 \mathrm{~Hz}, 0.3 \mathrm{H}), 7.04-7.01(\mathrm{~m}, 1.4 \mathrm{H}), 6.98(\mathrm{~d}, J=7.3 \mathrm{~Hz}$, $0.3 \mathrm{H}), 3.93(\mathrm{~s}, 2.1 \mathrm{H}), 3.92(\mathrm{~s}, 0.9 \mathrm{H}) \mathrm{ppm} ;{ }^{13} \mathrm{C} \mathrm{NMR}\left(150 \mathrm{MHz}, \mathrm{CDCl}_{3}\right) \delta 157.9$, 156.0, 147.5, 146.6, 144.8, 138.2, 131.3, 131.1, 129.7, 129.5, 128.1, 128.0, 127.0, 126.9, 124.7, 124.6, 124.5, 123.9, 123.4, 121.2, 121.1, 120.4, 114.4, 112.3, 111.1, 110.2, 101.3, 93.1, 56.0, $55.7 \mathrm{ppm}$.

3.4 10-Fluorobenzo[4,5] imidazo[2,1-a]isoquinoline and 9-fluorobenzo [4,5]imidazo[2,1-a]isoquinoline $(2 \boldsymbol{d})^{[2]}$

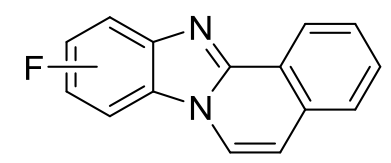

Yellow solid (96.8 mg, 82\% yield). M.p. $148-152{ }^{\circ} \mathrm{C} ;{ }^{1} \mathrm{H}$ NMR $\left(400 \mathrm{MHz}, \mathrm{CDCl}_{3}\right) \delta$ $8.77-8.73(\mathrm{~m}, 1 \mathrm{H}), 8.08(\mathrm{~d}, J=7.3 \mathrm{~Hz}, 0.6 \mathrm{H}), 8.00(\mathrm{~d}, J=7.4 \mathrm{~Hz}, 0.4 \mathrm{H}), 7.91$ (dd, $J$ $=8.9,4.8 \mathrm{~Hz}, 0.4 \mathrm{H}), 7.73-7.62(\mathrm{~m}, 4 \mathrm{H}), 7.47(\mathrm{dd}, J=8.3,2.3 \mathrm{~Hz}, 0.6 \mathrm{H}), 7.23(\mathrm{dd}, J$ $=9.0,2.4 \mathrm{~Hz}, 0.4 \mathrm{H}), 7.13(\mathrm{td}, J=9.2,2.4 \mathrm{~Hz}, 0.6 \mathrm{H}), 7.06(\mathrm{~d}, J=7.3 \mathrm{~Hz}, 0.6 \mathrm{H}), 7.03$ 
$(\mathrm{d}, J=7.3 \mathrm{~Hz}, 0.4 \mathrm{H}) \mathrm{ppm} ;{ }^{13} \mathrm{C} \mathrm{NMR}\left(150 \mathrm{MHz}, \mathrm{CDCl}_{3}\right) \delta 160.8(\mathrm{~d}, J=238.5 \mathrm{~Hz})$, $158.8(\mathrm{~d}, J=240.0 \mathrm{~Hz}), 148.5,131.5,131.4,130.2,130.1,128.4,128.3,127.1,127.0$, 126.7, 125.0, 124.8, 123.6, 123.2, 121.2, 121.1, $120.6(\mathrm{~d}, J=9 \mathrm{~Hz}), 113.3,113.2$, 111.7, 111.6, 110.4 (d, $J=22.5 \mathrm{~Hz}), 110.3$ (d, $J=7.5 \mathrm{~Hz}), 110.0,105.4(\mathrm{~d}, J=24.0$ $\mathrm{Hz}), 96.7,96.5 \mathrm{ppm} ;{ }^{19} \mathrm{~F} \mathrm{NMR}\left(376 \mathrm{MHz}, \mathrm{CDCl}_{3}\right) \delta-117.3,-119.1 \mathrm{ppm}$.

3.5 10-Chlorobenzo[4,5]imidazo[2,1-a]isoquinoline and 9-chlorobenzo [4,5]imidazo[2,1-a]isoquinoline $(2 \boldsymbol{e})^{[2]}$

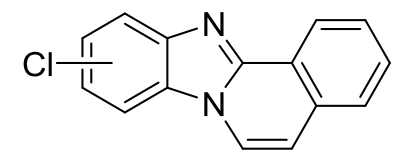

Yellow solid (110.9 mg, 88\% yield). M.p. $161-175{ }^{\circ} \mathrm{C} ;{ }^{1} \mathrm{H}$ NMR (400 MHz, $\mathrm{CDCl}_{3}$ ) $\delta 8.72(\mathrm{t}, J=4.6 \mathrm{~Hz}, 1 \mathrm{H}), 7.99(\mathrm{~d}, J=7.3 \mathrm{~Hz}, 0.58 \mathrm{H}), 7.95-7.91(\mathrm{~m}, 1 \mathrm{H}), 7.86(\mathrm{~d}, J$ $=8.7 \mathrm{~Hz}, 0.42 \mathrm{H}), 7.71-7.61(\mathrm{~m}, 4 \mathrm{H}), 7.42(\mathrm{dd}, J=8.7,1.9 \mathrm{~Hz}, 0.42 \mathrm{H}), 7.28(\mathrm{dd}, J=$ 8.6, $1.9 \mathrm{~Hz}, 0.58 \mathrm{H}), 6.99(\mathrm{t}, J=6.5 \mathrm{~Hz}, 1 \mathrm{H}) \mathrm{ppm} ;{ }^{13} \mathrm{C} \mathrm{NMR}\left(150 \mathrm{MHz}, \mathrm{CDCl}_{3}\right) \delta$ $148.1,147.8,144.4,142.2$, 131.5, 131.4, 130.4, 130.3, 130.2, 130.1, 128.5, 128.4, $128.3,127.4$, 127.2, 127.1, 125.3, 124.9, 124.8, 123.3, 123.1, 122.2, 121.0, 120.9, $120.6,119.4,111.9,111.8,110.4,109.9$ ppm.

3.6 10-Bromobenzo[4,5]imidazo[2,1-a]isoquinoline and 9-bromobenzo [4,5]imidazo[2,1-a]isoquinoline $(2 f)$

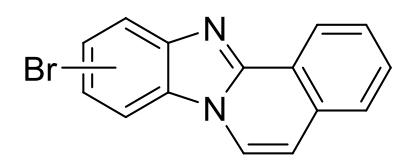

Yellow solid (115.4 mg, 78\% yield). M.p. $187-192{ }^{\circ} \mathrm{C} ;{ }^{1} \mathrm{H}$ NMR (400 MHz, $\mathrm{CDCl}_{3}$ ) $\delta 8.74(\mathrm{dd}, J=5.7,3.4 \mathrm{~Hz}, 1 \mathrm{H}), 8.10(\mathrm{~d}, J=1.7 \mathrm{~Hz}, 0.78 \mathrm{H}), 8.04(\mathrm{~d}, J=7.3 \mathrm{~Hz}$, $0.78 \mathrm{H}), 8.00(\mathrm{~d}, J=7.3 \mathrm{~Hz}, 0.22 \mathrm{H}), 7.92(\mathrm{~d}, J=1.7 \mathrm{~Hz}, 0.22 \mathrm{H}), 7.83(\mathrm{~d}, J=8.6 \mathrm{~Hz}$, $0.22 \mathrm{H}), 7.73-7.55(\mathrm{~m}, 4 \mathrm{H}), 7.44(\mathrm{dd}, J=8.6,1.7 \mathrm{~Hz}, 0.78 \mathrm{H}), 7.06-7.03(\mathrm{~m}, 1 \mathrm{H})$ ppm; ${ }^{13} \mathrm{C}$ NMR (150 MHz, $\left.\mathrm{CDCl}_{3}\right) \delta 148.0,144.9,131.6,131.5,130.4,130.3,128.9$, $128.5,128.4$, 128.0, 127.2, 127.1, 125.0, 124.9, 124.8, 123.2, 122.6, 121.0, 120.9, 117.8, 114.7, 113.0, 112.0, 111.9, $110.9 \mathrm{ppm}$; HRMS (ESI): $\mathrm{m} / z(\mathrm{M}+\mathrm{H})^{+}$calcd for $\mathrm{C}_{15} \mathrm{H}_{10} \mathrm{BrN}_{2}$ : 297.0022; Found: 297.0022. 9-(trifluoromethyl)benzo[4,5]imidazo[2,1-a]isoquinoline (2g) 


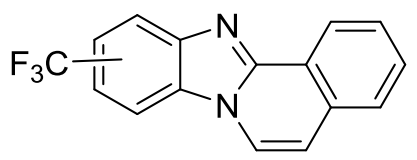

White solid (111.6 mg, 78\% yield). M.p. $182-189{ }^{\circ} \mathrm{C} ;{ }^{1} \mathrm{H}$ NMR $\left(400 \mathrm{MHz}, \mathrm{CDCl}_{3}\right) \delta$ $8.82-8.78(\mathrm{~m}, 1 \mathrm{H}), 8.28(\mathrm{~s}, 0.64 \mathrm{H}), 8.19(\mathrm{~d}, J=7.3 \mathrm{~Hz}, 0.36 \mathrm{H}), 8.15(\mathrm{~d}, J=7.3 \mathrm{~Hz}$, $0.64 \mathrm{H}), 8.12(\mathrm{~s}, 0.36 \mathrm{H}), 8.07(\mathrm{~d}, J=8.6 \mathrm{~Hz}, 0.36 \mathrm{H}), 7.89$ (d, $J=8.5 \mathrm{~Hz}, 0.64 \mathrm{H}), 7.79$ $-7.62(\mathrm{~m}, 4 \mathrm{H}), 7.16(\mathrm{~d}, J=7.2 \mathrm{~Hz}, 0.36 \mathrm{H}), 7.13(\mathrm{~d}, J=7.2 \mathrm{~Hz}, 0.64 \mathrm{H}) \mathrm{ppm} ;{ }^{13} \mathrm{C}$ NMR $\left(150 \mathrm{MHz}, \mathrm{CDCl}_{3}\right) \delta 148.7,143.1,131.8,131.7,131.6,130.7,130.6,128.6$, $127.3,127.2$, 127.1, 126.9, 125.5, 125.2, 125.1, 123.7, 123.2, 121.0120 .9 , 120.1, $118.5(\mathrm{q}, J=10.5 \mathrm{~Hz}), 117.4(\mathrm{q}, J=12.0 \mathrm{~Hz}), 112.3,110.3 \mathrm{ppm} ;{ }^{19} \mathrm{~F}$ NMR $(376 \mathrm{MHz}$, $\left.\mathrm{CDCl}_{3}\right) \delta$-60.7, -61.2 ppm; HRMS (ESI): $\mathrm{m} / z(\mathrm{M}+\mathrm{H})^{+}$calcd for $\mathrm{C}_{16} \mathrm{H}_{10} \mathrm{~F}_{3} \mathrm{~N}_{2}: 287.0791$; Found: 287.0789.

3.8 3-Methylbenzo[4,5]imidazo[2,1-a]isoquinoline $(\mathbf{2 h})^{[3]}$<smiles>Cc1ccc2c(ccn3c4ccccc4nc23)c1</smiles>

Yellow solid (89.4 mg, 77\% yield). M.p. $124-125{ }^{\circ} \mathrm{C} .{ }^{1} \mathrm{H}$ NMR $\left(400 \mathrm{MHz}, \mathrm{CDCl}_{3}\right) \delta$ $8.67(\mathrm{~d}, J=8.8 \mathrm{~Hz}, 1 \mathrm{H}), 8.06(\mathrm{~d}, J=7.2 \mathrm{~Hz}, 1 \mathrm{H}), 7.99(\mathrm{~d}, J=8.2 \mathrm{~Hz}, 1 \mathrm{H}), 7.76(\mathrm{~d}, J$ $=8.1 \mathrm{~Hz}, 1 \mathrm{H}), 7.51-7.46(\mathrm{~m}, 3 \mathrm{H}), 7.37(\mathrm{t}, J=7.6 \mathrm{~Hz}, 1 \mathrm{H}), 6.94(\mathrm{~d}, J=7.2 \mathrm{~Hz}, 1 \mathrm{H})$, $2.51(\mathrm{~s}, 3 \mathrm{H}) \mathrm{ppm} ;{ }^{13} \mathrm{C} \mathrm{NMR}\left(150 \mathrm{MHz}, \mathrm{CDCl}_{3}\right) \delta 147.4,143.8,140.3,131.7,130.0$, $129.7,126.9,124.8,124.5,121.6,121.3,121.2,119.7,111.2,109.7,21.8$ ppm.

3.9 2-Methoxybenzo[4,5]imidazo[2,1-a]isoquinoline $(2 i)^{[4]}$

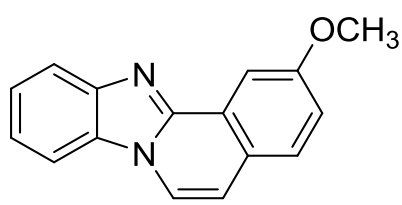

White solid (104.2 mg, 84\% yield). M.p. $111-113{ }^{\circ} \mathrm{C} .{ }^{1} \mathrm{H}$ NMR $\left(400 \mathrm{MHz}, \mathrm{CDCl}_{3}\right) \delta$ $8.17(\mathrm{~d}, J=2.6 \mathrm{~Hz}, 1 \mathrm{H}), 8.03(\mathrm{dd}, J=13.5,7.7 \mathrm{~Hz}, 2 \mathrm{H}), 7.82(\mathrm{~d}, J=8.1 \mathrm{~Hz}, 1 \mathrm{H})$, $7.64(\mathrm{~d}, J=8.7 \mathrm{~Hz}, 1 \mathrm{H}), 7.53-7.48(\mathrm{~m}, 1 \mathrm{H}), 7.41-7.37(\mathrm{~m}, 1 \mathrm{H}), 7.26(\mathrm{dd}, J=8.7$, $2.7 \mathrm{~Hz}, 1 \mathrm{H}), 7.01(\mathrm{~d}, J=7.2 \mathrm{~Hz}, 1 \mathrm{H}), 4.02(\mathrm{~s}, 3 \mathrm{H}) \mathrm{ppm} ;{ }^{13} \mathrm{C} \mathrm{NMR}\left(150 \mathrm{MHz}, \mathrm{CDCl}_{3}\right)$ $\delta 159.6,147.1,143.7,130.1,128.6,125.6,124.8,124.7,121.7,120.9,119.7,119.1$, $111.1,109.9,105.1,55.9 \mathrm{ppm}$. 


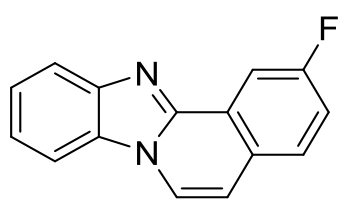

White solid (99.2 mg, 84\% yield). M.p. $168-170{ }^{\circ} \mathrm{C} .{ }^{1} \mathrm{H}$ NMR $\left(400 \mathrm{MHz}, \mathrm{CDCl}_{3}\right) \delta$ $8.40(\mathrm{dd}, J=9.3,2.6 \mathrm{~Hz}, 1 \mathrm{H}), 8.04(\mathrm{~d}, J=7.3 \mathrm{~Hz}, 1 \mathrm{H}), 7.99(\mathrm{dt}, J=8.2,0.9 \mathrm{~Hz}, 1 \mathrm{H})$, $7.76(\mathrm{dt}, J=8.2,0.9 \mathrm{~Hz}, 1 \mathrm{H}), 7.66(\mathrm{dd}, J=8.7,5.3 \mathrm{~Hz}, 1 \mathrm{H}), 7.52-7.48(\mathrm{~m}, 1 \mathrm{H}), 7.41$ $-7.31(\mathrm{~m}, 2 \mathrm{H}), 6.97(\mathrm{~d}, J=7.3 \mathrm{~Hz}, 1 \mathrm{H}) \mathrm{ppm} ;{ }^{13} \mathrm{C} \mathrm{NMR}\left(150 \mathrm{MHz}, \mathrm{CDCl}_{3}\right) \delta 162.0(\mathrm{~d}$, $J=249.0 \mathrm{~Hz}), 146.3(\mathrm{~d}, J=4.5 \mathrm{~Hz}), 143.6,130.0,129.3(\mathrm{~d}, J=9.0 \mathrm{~Hz}), 128.0(\mathrm{~d}, J$ $=3.0 \mathrm{~Hz}) 125.1(\mathrm{~d}, J=9.0 \mathrm{~Hz}), 124.8,122.2,120.6(\mathrm{~d}, J=3.0 \mathrm{~Hz}), 120.0,118.5(\mathrm{~d}, J$ $=24.0 \mathrm{~Hz}), 110.5,110.3(\mathrm{~d}, J=22.5 \mathrm{~Hz}), 109.9 \mathrm{ppm} ;{ }^{19} \mathrm{~F} \mathrm{NMR}\left(376 \mathrm{MHz}, \mathrm{CDCl}_{3}\right) \delta$ $-110.9 \mathrm{ppm}$.

\subsection{2-Chlorobenzo[4,5]imidazo[2,1-a]isoquinoline $(2 \boldsymbol{k})^{[5]}$}

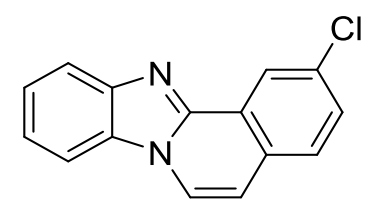

Yellow solid (107.2 mg, 85\% yield). M.p. $192-194{ }^{\circ} \mathrm{C} .{ }^{1} \mathrm{H}$ NMR (400 MHz, $\left.\mathrm{CDCl}_{3}\right)$ $\delta 8.76(\mathrm{~d}, J=2.0 \mathrm{~Hz}, 1 \mathrm{H}), 8.10(\mathrm{~d}, J=7.3 \mathrm{~Hz}, 1 \mathrm{H}), 7.99$ (d, $J=8.2 \mathrm{~Hz}, 1 \mathrm{H}), 7.79$ (d, $J=8.1 \mathrm{~Hz}, 1 \mathrm{H}), 7.63-7.49(\mathrm{~m}, 3 \mathrm{H}), 7.43-7.38(\mathrm{~m}, 1 \mathrm{H}), 6.99(\mathrm{~d}, J=7.2 \mathrm{~Hz}, 1 \mathrm{H})$ ppm; ${ }^{13} \mathrm{C}$ NMR $\left(150 \mathrm{MHz}, \mathrm{CDCl}_{3}\right) \delta 146.0,143.7,134.1,130.3,130.0,129.7,128.4$, $124.9,124.6,124.4,122.2,121.6,120.0,110.5,109.9$ ppm.

\subsection{2-Methoxy-10-methylbenzo[4,5]imidazo[2,1-a]isoquinoline (2l)}<smiles>COc1ccc2ccn3c4ccc(C)cc4nc3c2c1</smiles>

Yellow solid (111.4 mg, 85\% yield). M.p. $164-166{ }^{\circ} \mathrm{C} .{ }^{1} \mathrm{H}$ NMR (400 MHz, $\left.\mathrm{CDCl}_{3}\right)$ $\delta 8.17(\mathrm{~d}, J=2.5 \mathrm{~Hz}, 1 \mathrm{H}), 8.05(\mathrm{~d}, J=7.2 \mathrm{~Hz}, 1 \mathrm{H}), 7.79(\mathrm{~s}, 1 \mathrm{H}), 7.70(\mathrm{~d}, J=8.3 \mathrm{~Hz}$, $1 \mathrm{H}), 7.65(\mathrm{~d}, J=8.7 \mathrm{~Hz}, 1 \mathrm{H}), 7.26-7.19(\mathrm{~m}, 2 \mathrm{H}), 7.01(\mathrm{~d}, J=7.2 \mathrm{~Hz}, 1 \mathrm{H}), 4.03$ (s, 3H), 2.58 (s, 3H) ppm; $\left.{ }^{13} \mathrm{C} \mathrm{NMR} \mathrm{(150} \mathrm{MHz,} \mathrm{CDCl}_{3}\right) \delta$ 159.6, 147.1, 144.0, 134.6, 
128.6, 128.3, 125.6, 124.9, 123.4, 120.5, 119.4, 119.2, 110.9, 109.4, 105.0, 55.9, 21.9 ppm; HRMS (ESI): $m / z(\mathrm{M}+\mathrm{H})^{+}$calcd for $\mathrm{C}_{17} \mathrm{H}_{15} \mathrm{~N}_{2} \mathrm{O}$ : 263.1179; Found: 263.1179 .

3.13 2-Fluoro-10-methylbenzo[4,5]imidazo[2,1-a]isoquinoline and 2-fluoro-9-methylbenzo[4,5]imidazo[2,1-a]isoquinoline (2m)

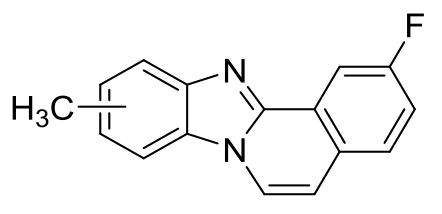

White solid (100.0 mg, 80\% yield). M.p. $135-141{ }^{\circ} \mathrm{C} ;{ }^{1} \mathrm{H}$ NMR (400 MHz, $\left.\mathrm{CDCl}_{3}\right) \delta$ $8.38-8.33(\mathrm{~m}, 1 \mathrm{H}), 7.97-7.91(\mathrm{~m}, 1 \mathrm{H}), 7.83(\mathrm{~d}, J=8.3 \mathrm{~Hz}, 0.44 \mathrm{H}), 7.73(\mathrm{~s}, 0.56 \mathrm{H})$, $7.66-7.56$ (m, 2H), 7.49 (s, 0.44H), $7.33-7.27$ (m, 1H), 7.17 (d, $J=8.3 \mathrm{~Hz}, 0.56 \mathrm{H})$, $6.90(\mathrm{td}, J=7.5,2.0 \mathrm{~Hz}, 1 \mathrm{H}), 2.55(\mathrm{~s}, 1.68 \mathrm{H}), 2.55(\mathrm{~s}, 1.32 \mathrm{H}) \mathrm{ppm} ;{ }^{13} \mathrm{C} \mathrm{NMR}(150$ $\left.\mathrm{MHz}, \mathrm{CDCl}_{3}\right) \delta 162.0(\mathrm{~d}, J=247.5 \mathrm{~Hz}), 161.9(\mathrm{~d}, J=247.5 \mathrm{~Hz}), 146.2(\mathrm{~d}, J=3.0$ $\mathrm{Hz}), 145.9(\mathrm{~d}, J=4.5 \mathrm{~Hz}), 143.9,141.7,134.8,132.3,130.1,129.2(\mathrm{~d}, J=9.0 \mathrm{~Hz})$, 128.1, 127.9 (d, $J=1.5 \mathrm{~Hz}), 127.8(\mathrm{~d}, J=3.0 \mathrm{~Hz}), 126.5,125.2(\mathrm{~d}, J=10.5 \mathrm{~Hz})$, $125.0(\mathrm{~d}, J=10.5 \mathrm{~Hz}), 123.8,120.6(\mathrm{~d}, J=3.0 \mathrm{~Hz}), 120.5(\mathrm{~d}, J=1.5 \mathrm{~Hz}), 119.5$, $119.4,118.3$ (d, $J=22.5 \mathrm{~Hz}), 118.2(\mathrm{~d}, J=22.5 \mathrm{~Hz}), 110.3,110.2(\mathrm{~d}, J=30.0 \mathrm{~Hz})$, $110.1(\mathrm{~d}, J=24.0 \mathrm{~Hz}), 109.6,109.3,22.0,21.9 \mathrm{ppm} ;{ }^{19} \mathrm{~F} \mathrm{NMR}\left(376 \mathrm{MHz}, \mathrm{CDCl}_{3}\right) \delta$ -111.2 ppm; HRMS (ESI): $m / z(\mathrm{M}+\mathrm{H})^{+}$calcd for $\mathrm{C}_{16} \mathrm{H}_{12} \mathrm{FN}_{2:}$ 251.0979; Found: 251.0979 .

\subsection{2-Chloro-10-methylbenzo[4,5]imidazo[2,1-a]isoquinoline (2n)}<smiles>Cc1ccc2c(c1)nc1c3cc(Cl)ccc3ccn21</smiles>

White solid (106.4 mg, 80\% yield). M.p. $180-182{ }^{\circ} \mathrm{C} .{ }^{1} \mathrm{H} \mathrm{NMR}\left(400 \mathrm{MHz}, \mathrm{CDCl}_{3}\right) \delta$ $8.75(\mathrm{~d}, J=2.0 \mathrm{~Hz}, 1 \mathrm{H}), 8.08(\mathrm{~d}, J=7.3 \mathrm{~Hz}, 1 \mathrm{H}), 7.76(\mathrm{~s}, 1 \mathrm{H}), 7.70-7.52(\mathrm{~m}, 3 \mathrm{H})$, $7.23(\mathrm{~d}, J=8.3 \mathrm{~Hz}, 1 \mathrm{H}), 6.97(\mathrm{~d}, J=7.2 \mathrm{~Hz}, 1 \mathrm{H}), 2.58(\mathrm{~s}, 3 \mathrm{H}) \mathrm{ppm} ;{ }^{13} \mathrm{C}$ NMR $(150$ $\left.\mathrm{MHz}, \mathrm{CDCl}_{3}\right) \delta 146.0,144.1,134.9,134.0,130.1,129.7,128.4,128.1,124.6,124.3$, 123.9, 121.6, 119.7, 110.2, 109.3, 21.9 ppm; HRMS (ESI): $\mathrm{m} / \mathrm{z}(\mathrm{M}+\mathrm{H})^{+}$calcd for $\mathrm{C}_{16} \mathrm{H}_{12} \mathrm{ClN}_{2:}$ 267.0684; Found: 267.0684.

3.15 2,10-Dimethoxybenzo[4,5]imidazo[2,1-a]isoquinoline (2o) 
<smiles>COc1ccc2c(c1)nc1c3cc(OC)ccc3ccn21</smiles>

Yellow solid (102.9 mg, 74\% yield). M.p. $134-135{ }^{\circ} \mathrm{C} .{ }^{1} \mathrm{H}$ NMR (400 MHz, $\left.\mathrm{CDCl}_{3}\right)$ $\delta 8.12(\mathrm{~d}, J=2.1 \mathrm{~Hz}, 1 \mathrm{H}), 7.96(\mathrm{dd}, J=7.3,1.3 \mathrm{~Hz}, 1 \mathrm{H}), 7.89(\mathrm{dd}, J=8.9,1.0 \mathrm{~Hz}$, $1 \mathrm{H}), 7.62(\mathrm{~d}, J=8.7 \mathrm{~Hz}, 1 \mathrm{H}), 7.25-7.21(\mathrm{~m}, 2 \mathrm{H}), 7.15(\mathrm{ddd}, J=8.9,2.4,1.4 \mathrm{~Hz}, 1 \mathrm{H})$, $6.97(\mathrm{~d}, J=7.3 \mathrm{~Hz}, 1 \mathrm{H}), 4.01$ (s, 3H), 3.94 (s, 3H).ppm; ${ }^{13} \mathrm{C} \mathrm{NMR}\left(150 \mathrm{MHz}, \mathrm{CDCl}_{3}\right)$ $\delta 159.6,156.0,146.6,138.1,130.4,128.6,125.2,125.1,120.3,120.2,118.9,114.5$, 111.0, 104.8, 93.2, 56.0, 55.9 ppm; HRMS (ESI): $m / z(\mathrm{M}+\mathrm{H})^{+}$calcd for $\mathrm{C}_{17} \mathrm{H}_{15} \mathrm{~N}_{2} \mathrm{O}_{2}$ : 279.1128; Found: 279.1128.

\subsection{2-Chloro-10-methoxybenzo[4,5]imidazo[2,1-a]isoquinoline (2p)}

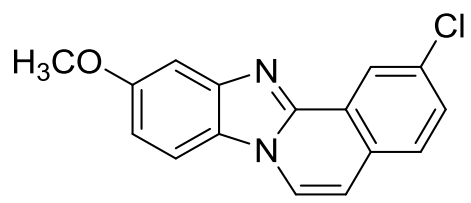

White solid (122.7 mg, 87\% yield). M.p. $201-203{ }^{\circ} \mathrm{C} .{ }^{1} \mathrm{H}$ NMR $\left(400 \mathrm{MHz}, \mathrm{CDCl}_{3}\right) \delta$ $8.75-8.70(\mathrm{~m}, 1 \mathrm{H}), 8.05(\mathrm{~d}, J=7.2 \mathrm{~Hz}, 1 \mathrm{H}), 7.69-7.51(\mathrm{~m}, 3 \mathrm{H}), 7.41(\mathrm{~d}, J=2.1 \mathrm{~Hz}$, $1 \mathrm{H}), 7.07-6.94(\mathrm{~m}, 2 \mathrm{H}), 3.93(\mathrm{~s}, 3 \mathrm{H}) \mathrm{ppm} ;{ }^{13} \mathrm{C} \mathrm{NMR}\left(150 \mathrm{MHz}, \mathrm{CDCl}_{3}\right) \delta 158.1$, $146.2,144.8,134.0,130.0,129.4,128.3,124.6,124.4,124.2,121.5,112.8,110.3$, 101.3, 55.7 ppm; HRMS (ESI): $m / z(\mathrm{M}+\mathrm{H})^{+}$calcd for $\mathrm{C}_{16} \mathrm{H}_{12} \mathrm{ClN}_{2} \mathrm{O}: 283.0633$; Found: 283.0630 .

\subsection{9-Fluoro-2-methoxybenzo[4,5]imidazo[2,1-a]isoquinoline (2q)}<smiles>COc1ccc2ccn3c4cc(F)ccc4nc3c2c1</smiles>

White solid (101.1 mg, 76\% yield). M.p. $150-152{ }^{\circ} \mathrm{C} .{ }^{1} \mathrm{H}$ NMR $\left(400 \mathrm{MHz}, \mathrm{CDCl}_{3}\right) \delta$ $8.10(\mathrm{~d}, J=2.5 \mathrm{~Hz}, 1 \mathrm{H}), 7.98(\mathrm{~d}, J=7.2 \mathrm{~Hz}, 1 \mathrm{H}), 7.70(\mathrm{dd}, J=8.8,4.6 \mathrm{~Hz}, 1 \mathrm{H}), 7.67$ $-7.60(\mathrm{~m}, 2 \mathrm{H}), 7.27(\mathrm{~d}, J=2.7 \mathrm{~Hz}, 1 \mathrm{H}), 7.12(\mathrm{td}, J=9.0,2.4 \mathrm{~Hz}, 1 \mathrm{H}), 7.01(\mathrm{~d}, J=$ $7.2 \mathrm{~Hz}, 1 \mathrm{H}), 4.01$ (s, 3H) ppm; ${ }^{13} \mathrm{C} \mathrm{NMR}\left(150 \mathrm{MHz}, \mathrm{CDCl}_{3}\right) \delta 160.7(\mathrm{~d}, J=238.5$ $\mathrm{Hz}), 159.7,148.3,144.3(\mathrm{~d}, J=13.5 \mathrm{~Hz}), 128.6,126.8,125.5,124.5,120.9,118.9$, 111.5, $110.3(\mathrm{~d}, J=6.0 \mathrm{~Hz}), 110.2(\mathrm{~d}, J=43.5 \mathrm{~Hz}), 105.3(\mathrm{~d}, J=24.0 \mathrm{~Hz}), 105.0$, 
$55.9 \mathrm{ppm} ;{ }^{19} \mathrm{~F}$ NMR $\left(376 \mathrm{MHz}, \mathrm{CDCl}_{3}\right) \delta-117.4 \mathrm{ppm}$; HRMS (ESI): $m / z(\mathrm{M}+\mathrm{H})^{+}$ calcd for $\mathrm{C}_{16} \mathrm{H}_{12} \mathrm{FN}_{2} \mathrm{O}$ : 267.0928; Found: 267.0928.

3.18 10-Chloro-3-methylbenzo[4,5]imidazo[2,1-a]isoquinoline and 9-chloro-3methylbenzo[4,5]imidazo[2,1-a]isoquinoline (2r)

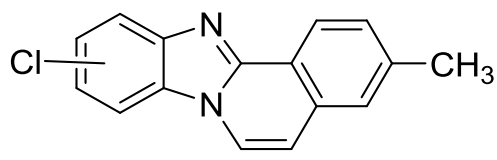

White solid (107.8 mg, 81\% yield). M.p. $181-190{ }^{\circ} \mathrm{C} ;{ }^{1} \mathrm{H}$ NMR $\left(400 \mathrm{MHz}, \mathrm{CDCl}_{3}\right) \delta$ $8.61(\mathrm{~d}, J=8.8 \mathrm{~Hz}, 1 \mathrm{H}), 8.02-7.83(\mathrm{~m}, 2 \mathrm{H}), 7.71(\mathrm{~d}, J=1.7 \mathrm{~Hz}, 0.24 \mathrm{H}), 7.63(\mathrm{~d}, J=$ $8.6 \mathrm{~Hz}, 0.76 \mathrm{H}), 7.48(\mathrm{~d}, J=6.9 \mathrm{~Hz}, 2 \mathrm{H}), 7.42(\mathrm{dd}, J=8.7,2.0 \mathrm{~Hz}, 0.24 \mathrm{H}), 7.29(\mathrm{dd}, J$ $=8.6,1.9 \mathrm{~Hz}, 0.76 \mathrm{H}), 6.94(\mathrm{t}, J=6.7 \mathrm{~Hz}, 1 \mathrm{H}), 2.53(\mathrm{~d}, J=2.8 \mathrm{~Hz}, 3 \mathrm{H}) \mathrm{ppm} ;{ }^{13} \mathrm{C}$ NMR $\left(150 \mathrm{MHz}, \mathrm{CDCl}_{3}\right) \delta 148.3,144.6,140.8,140.7,131.7,131.6,130.1,129.9$, $128.6,127.0,126.9,125.2$, 124.9, 124.8, 121.9, 121.0, 120.9, 120.4, 119.3, 111.7, 111.6, 110.4, 109.9, 21.8, 21.7 ppm; HRMS (ESI): $m / z(\mathrm{M}+\mathrm{H})^{+}$calcd for $\mathrm{C}_{16} \mathrm{H}_{12} \mathrm{ClN}_{2}$ : 267.0684; Found: 267.0683.

3.19 10-Chloro-2-methoxybenzo[4,5]imidazo[2,1-a]isoquinoline and 9-chloro-2methoxybenzo[4,5]imidazo[2,1-a]isoquinoline $(2 \mathrm{~s})$<smiles>COc1ccc2ccn3c4ccc(Cl)cc4nc3c2c1</smiles>

White solid (131.2 mg, 93\% yield). M.p. $177-185{ }^{\circ} \mathrm{C} ;{ }^{1} \mathrm{H}$ NMR (400 MHz, $\left.\mathrm{CDCl}_{3}\right) \delta$ $8.08(\mathrm{~s}, 0.6 \mathrm{H}), 8.03(\mathrm{~s}, 0.4 \mathrm{H}), 7.93-7.90(\mathrm{~m}, 1.2 \mathrm{H}), 7.86-7.84(\mathrm{~m}, 0.8 \mathrm{H}), 7.67-$ 7.54 (m, 2H), $7.31-7.21(\mathrm{~m}, 2 \mathrm{H}), 7.00-6.98(\mathrm{~m}, 0.6 \mathrm{H}), 6.96-6.91(\mathrm{~m}, 0.4 \mathrm{H}), 4.00$ $(\mathrm{s}, 1.8 \mathrm{H}), 3.98$ (s, 1.2H) ppm; ${ }^{13} \mathrm{C} \mathrm{NMR}\left(150 \mathrm{MHz}, \mathrm{CDCl}_{3}\right) \delta 159.8,159.7,148.0$, 147.9 , 144.4, 144.3, 130.2, 130.1, 128.7, 128.6, 125.6, 125.5, 124.5, 124.4, 122.2, 122.1, 121.0, 120.9, 119.4, 119.3, 118.8, 118.7, 111.6, 111.5, 110.6, 110.5, 105.1, 105.0, 55.9, 55.8 ppm; HRMS (ESI): $m / z(\mathrm{M}+\mathrm{H})^{+}$calcd for $\mathrm{C}_{16} \mathrm{H}_{12} \mathrm{ClN}_{2} \mathrm{O}$ : 283.0633; Found: 283.0632.

3.20 9-Bromo-2-methoxybenzo[4,5]imidazo[2,1-a]isoquinoline (2t) 
<smiles>COc1ccc2ccn3c4cc(Br)ccc4nc3c2c1</smiles>

Yellow solid (114.1 mg, 70\% yield). M.p. $197-198{ }^{\circ} \mathrm{C} .{ }^{1} \mathrm{H}$ NMR (400 MHz, $\left.\mathrm{CDCl}_{3}\right)$ $\delta 8.13-8.04(\mathrm{~m}, 2 \mathrm{H}), 7.92(\mathrm{dd}, J=7.2,1.0 \mathrm{~Hz}, 1 \mathrm{H}), 7.65-7.57(\mathrm{~m}, 2 \mathrm{H}), 7.42(\mathrm{dt}, J$ $=8.6,1.5 \mathrm{~Hz}, 1 \mathrm{H}), 7.24(\mathrm{dd}, J=2.6,1.3 \mathrm{~Hz}, 1 \mathrm{H}), 6.99(\mathrm{~d}, J=7.2 \mathrm{~Hz}, 1 \mathrm{H}), 4.00(\mathrm{~s}, 3 \mathrm{H})$ ppm; ${ }^{13} \mathrm{C} \mathrm{NMR}\left(150 \mathrm{MHz}, \mathrm{CDCl}_{3}\right) \delta 159.7,147.8,144.8,129.0,128.7,125.6,124.7$, 124.5, 122.5, 121.0, 118.8, 117.7, 111.6, 111.0, 105.1, 55.9 ppm; HRMS (ESI): $\mathrm{m} / z$ $(\mathrm{M}+\mathrm{H})^{+}$calcd for $\mathrm{C}_{16} \mathrm{H}_{12} \mathrm{BrN}_{2} \mathrm{O}$ : 327.0128; Found: 327.0127 .

3.21 2-Chloro-9-(trifluoromethyl)benzo[4,5]imidazo[2,1-a]isoquinoline (2u)<smiles>FC(F)(F)c1ccc2nc3c4cc(Cl)ccc4ccn3c2c1</smiles>

Yellow solid (115.2 mg, 72\% yield). M.p. $191-192{ }^{\circ} \mathrm{C} .{ }^{1} \mathrm{H}$ NMR (400 MHz, $\left.\mathrm{CDCl}_{3}\right)$ $\delta 8.77(\mathrm{~d}, J=2.1 \mathrm{~Hz}, 1 \mathrm{H}), 8.19(\mathrm{~d}, J=7.3 \mathrm{~Hz}, 1 \mathrm{H}), 8.13-8.11(\mathrm{~m}, 1 \mathrm{H}), 8.05(\mathrm{~d}, J=$ $8.6 \mathrm{~Hz}, 1 \mathrm{H}), 7.80-7.74(\mathrm{~m}, 1 \mathrm{H}), 7.70(\mathrm{~d}, J=8.5 \mathrm{~Hz}, 1 \mathrm{H}), 7.64(\mathrm{dd}, J=8.5,2.1 \mathrm{~Hz}$, $1 \mathrm{H}), 7.12(\mathrm{~d}, J=7.3 \mathrm{~Hz}, 1 \mathrm{H}) \mathrm{ppm} ;{ }^{13} \mathrm{C} \mathrm{NMR}\left(150 \mathrm{MHz}, \mathrm{CDCl}_{3}\right) \delta 148.0,145.7$, 134.7, 131.1, 130.0, 129.4, 128.6, 124.7, 124.4, 124.3, 124.2, 121.8, 121.3, 120.4, 111.6, 107.9 (q, $J=4.5 \mathrm{~Hz}) \mathrm{ppm} ;{ }^{19} \mathrm{~F} \mathrm{NMR}\left(376 \mathrm{MHz}, \mathrm{CDCl}_{3}\right) \delta-60.8 \mathrm{ppm}$; HRMS (ESI): $m / z(\mathrm{M}+\mathrm{H})^{+}$calcd for $\mathrm{C}_{16} \mathrm{H}_{9} \mathrm{ClF}_{3} \mathrm{~N}_{2:}$ 321.0401; Found: 321.0401 . 
4. ${ }^{1} \mathrm{H},{ }^{13} \mathrm{C}$ and ${ }^{19} \mathrm{~F}$ NMR spectra for products $2 \mathrm{a}-2 \mathrm{u}$
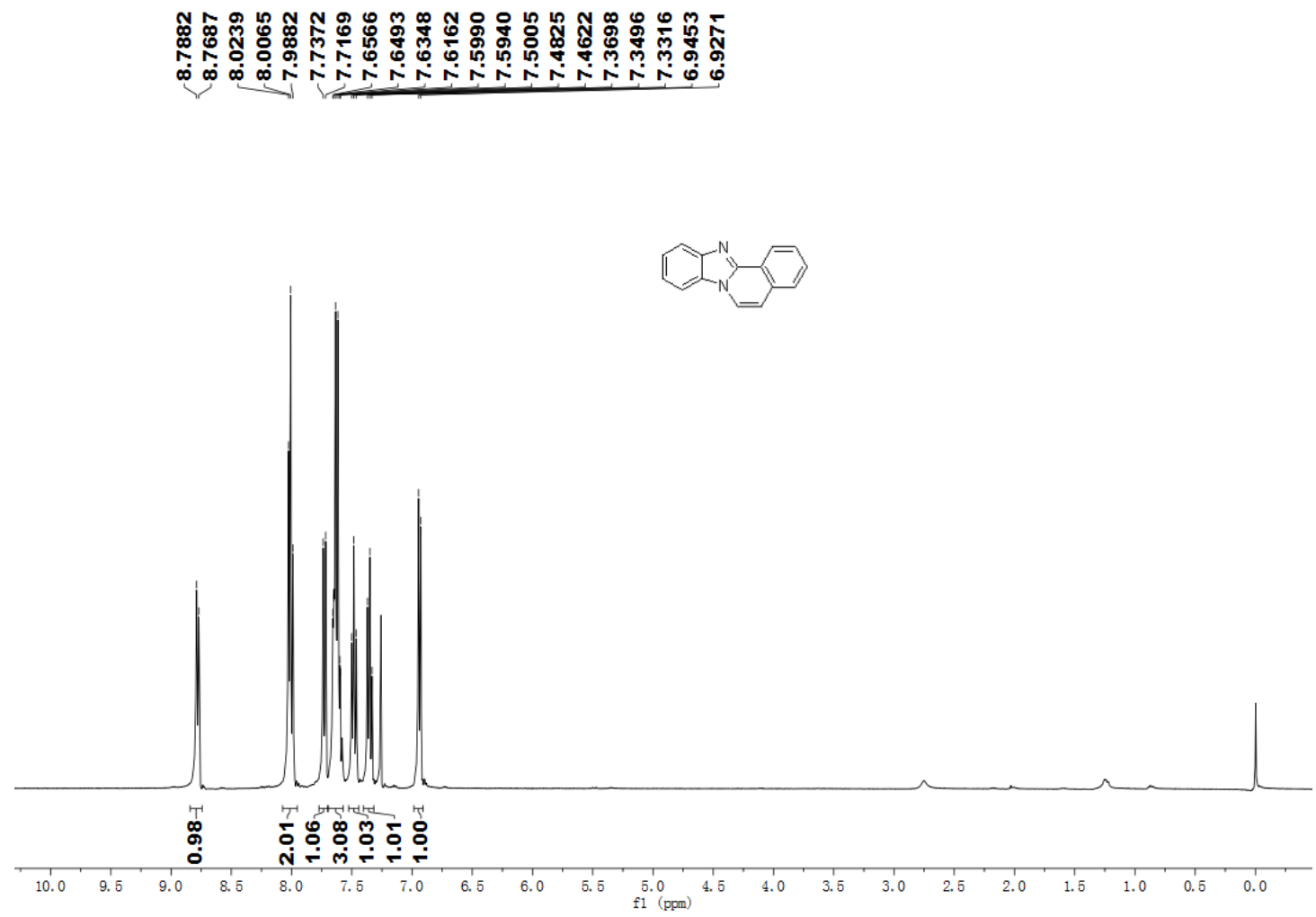

Fig S1. ${ }^{1} \mathrm{H}$ NMR (400 MHz, $\left.\mathrm{CDCl}_{3}\right)$ of benzo[4,5]imidazo[2,1-a]isoquinoline (2a)

\section{กำ}
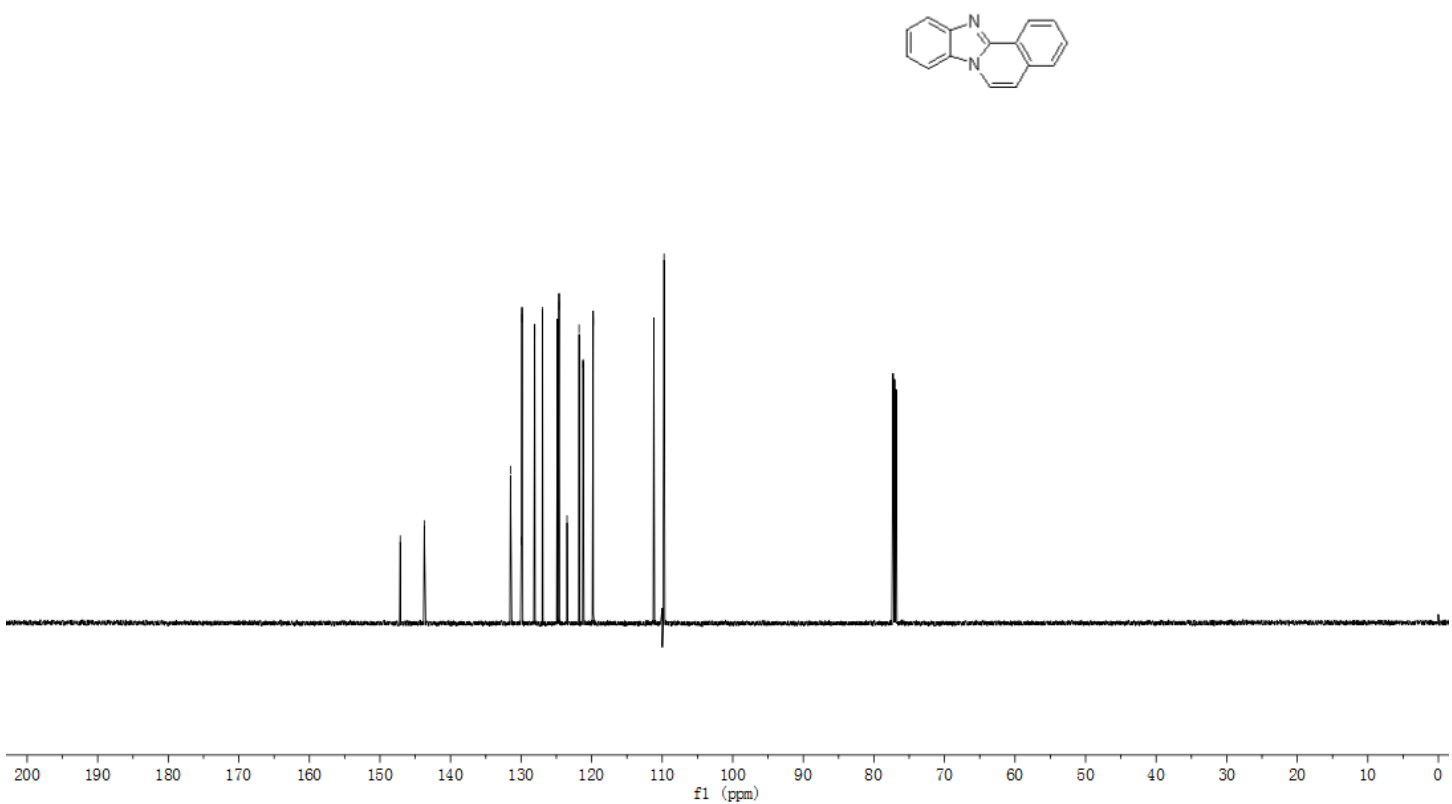

Fig S2. ${ }^{13} \mathrm{C}$ NMR $\left(150 \mathrm{MHz}, \mathrm{CDCl}_{3}\right)$ of benzo[4,5]imidazo[2,1-a]isoquinoline (2a) 

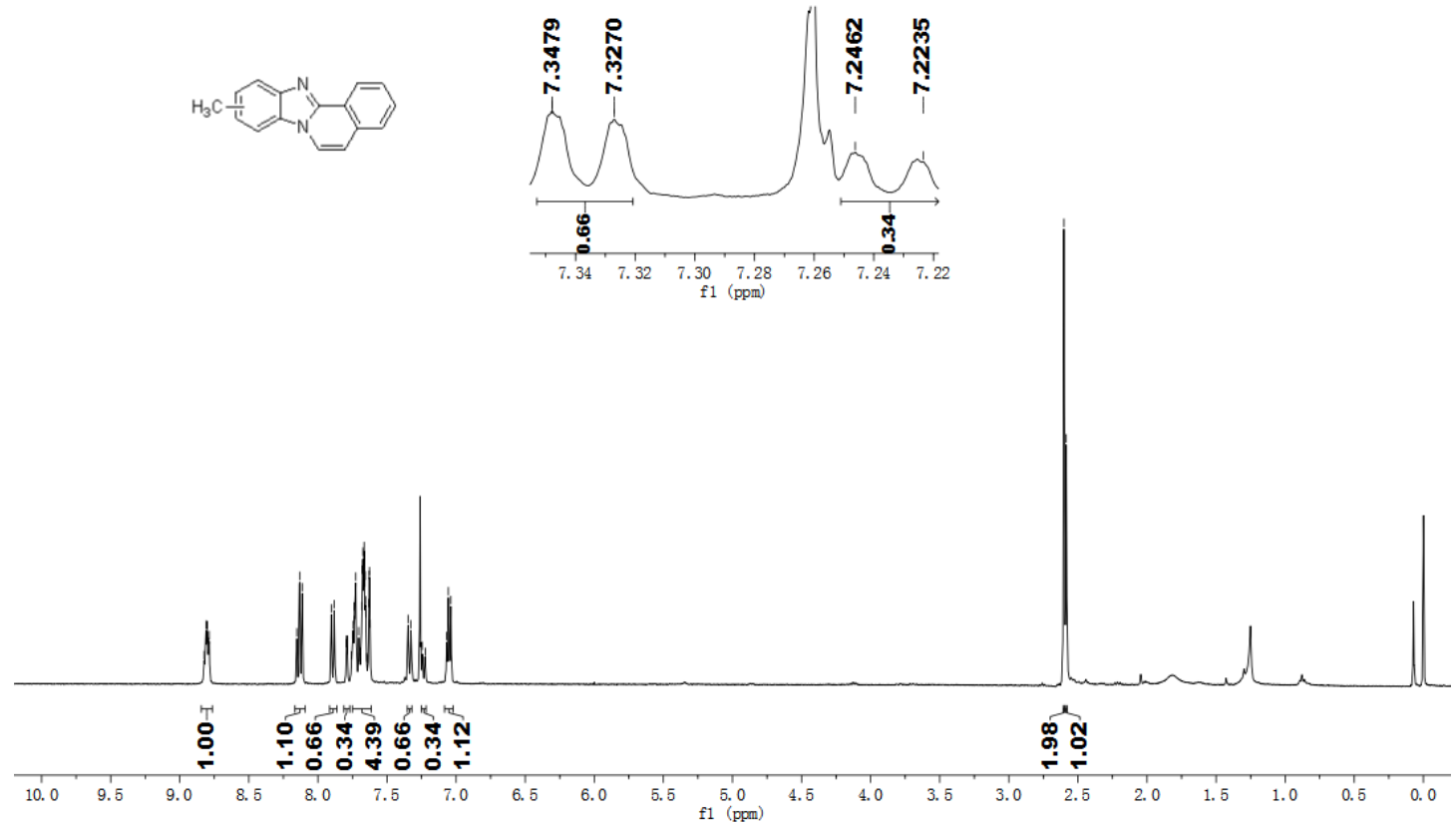

Fig S3. ${ }^{1} \mathrm{H}$ NMR (400 $\left.\mathrm{MHz}, \mathrm{CDCl}_{3}\right)$ of 10-methylbenzo[4,5]imidazo[2,1-a] isoquinoline and 9-methylbenzo[4,5]imidazo[2,1-a]isoquinoline (2b)

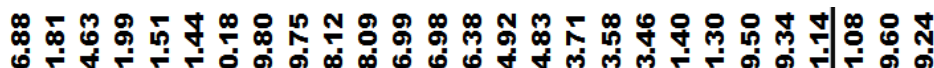

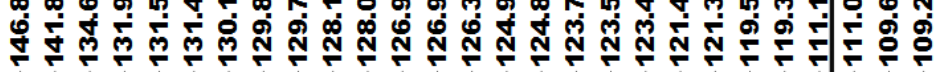

ลั่
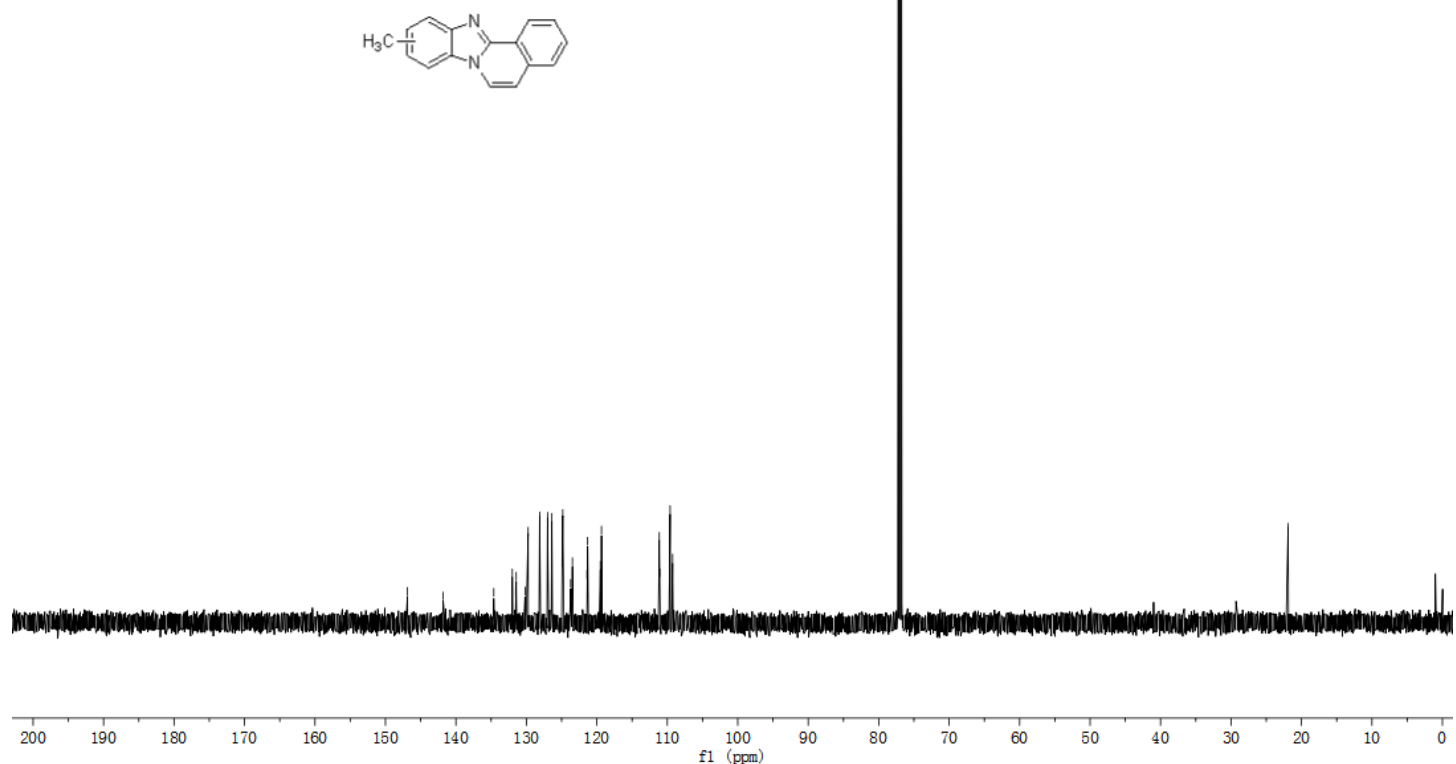

Fig S4. ${ }^{13} \mathrm{C} \mathrm{NMR}\left(150 \mathrm{MHz}, \mathrm{CDCl}_{3}\right)$ of 10 -methylbenzo[4,5]imidazo[2,1-a] isoquinoline and 9-methylbenzo[4,5]imidazo[2,1-a]isoquinoline (2b) 

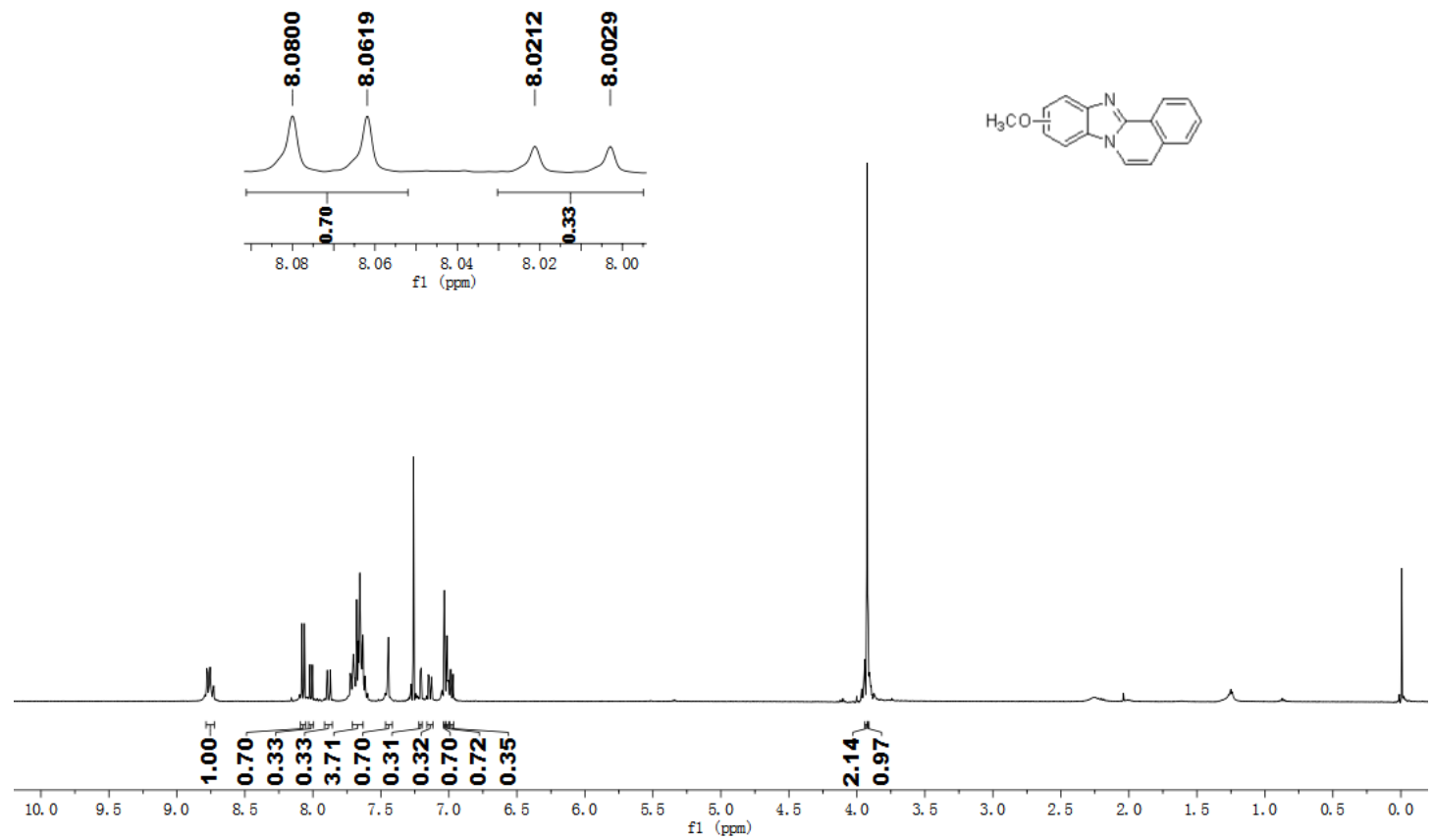

Fig S5. ${ }^{1} \mathrm{H}$ NMR $\left(400 \mathrm{MHz}, \mathrm{CDCl}_{3}\right)$ of 10-methoxybenzo[4,5]imidazo $[2,1-a]$ isoquinoline and 9-methoxybenzo[4,5]imidazo[2,1- $a$ ]isoquinoline (2c)

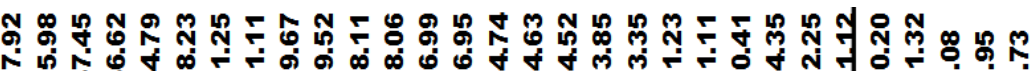

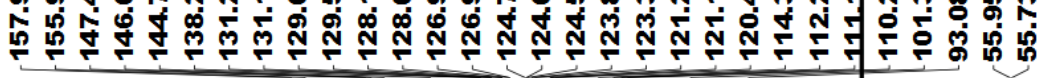
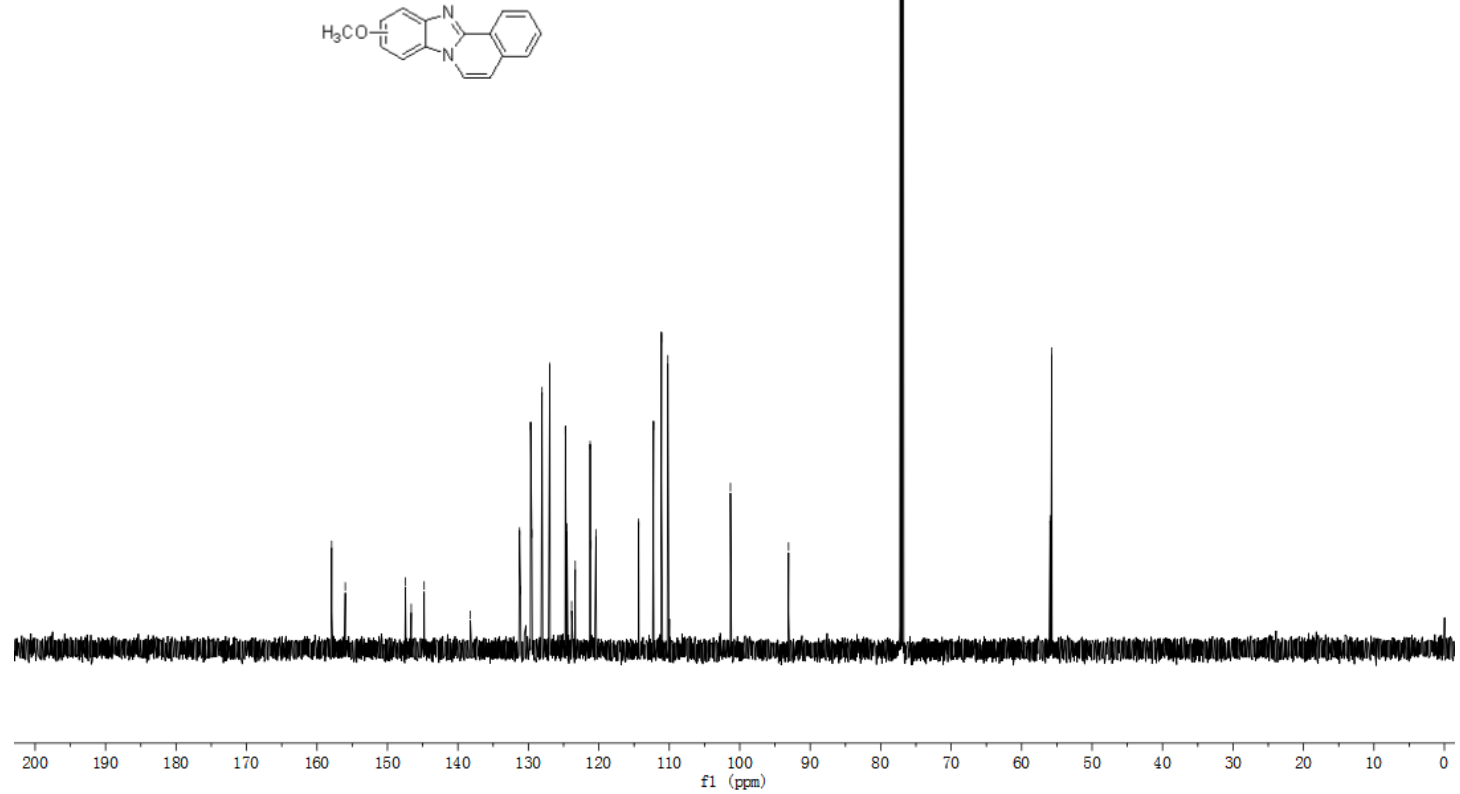

Fig S6. ${ }^{13} \mathrm{C} \mathrm{NMR}\left(150 \mathrm{MHz}, \mathrm{CDCl}_{3}\right)$ of 10 -methoxybenzo[4,5]imidazo[2,1-a] isoquinoline and 9-methoxybenzo[4,5]imidazo[2,1-a]isoquinoline (2c) 


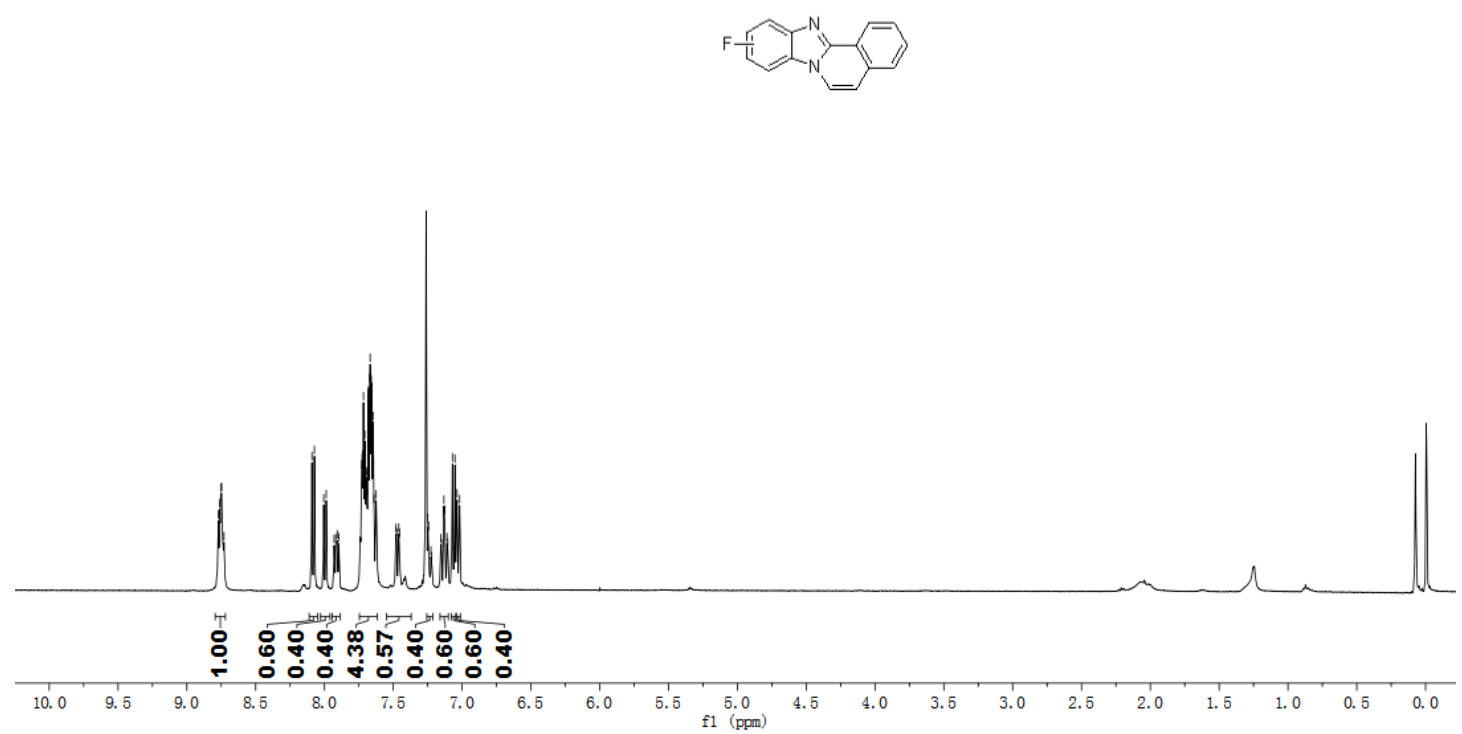

Fig S7. ${ }^{1} \mathrm{H}$ NMR $\left(400 \mathrm{MHz}, \mathrm{CDCl}_{3}\right)$ of 10 -fluorobenzo[4,5]imidazo[2,1-a] isoquinoline and 9-fluorobenzo[4,5]imidazo[2,1- $a$ ]isoquinoline(2d)

\section{mag the}

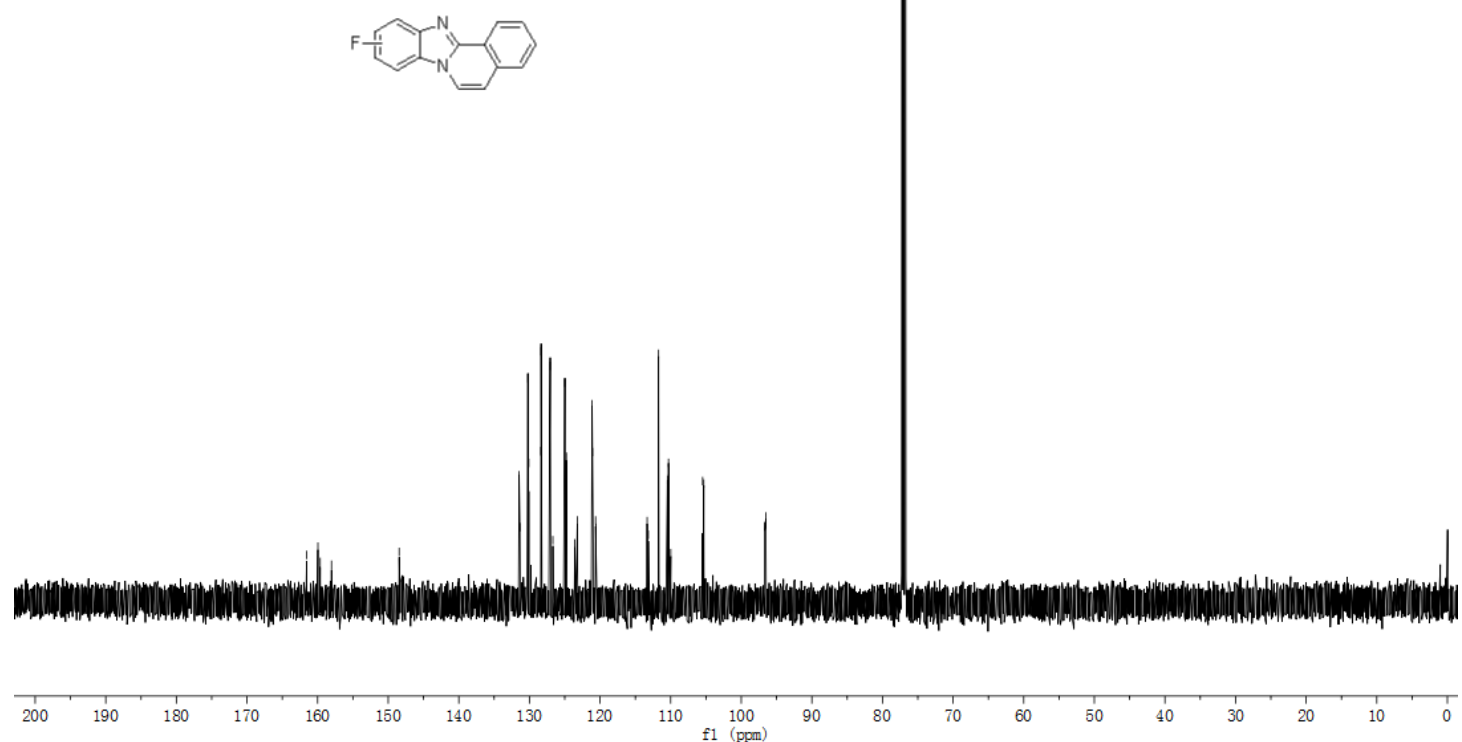

Fig S8. ${ }^{13} \mathrm{C}$ NMR $\left(150 \mathrm{MHz}, \mathrm{CDCl}_{3}\right)$ of 10 -fluorobenzo[4,5]imidazo[2,1- $a$ ] isoquinoline and 9-fluorobenzo[4,5]imidazo[2,1-a]isoquinoline(2d) 

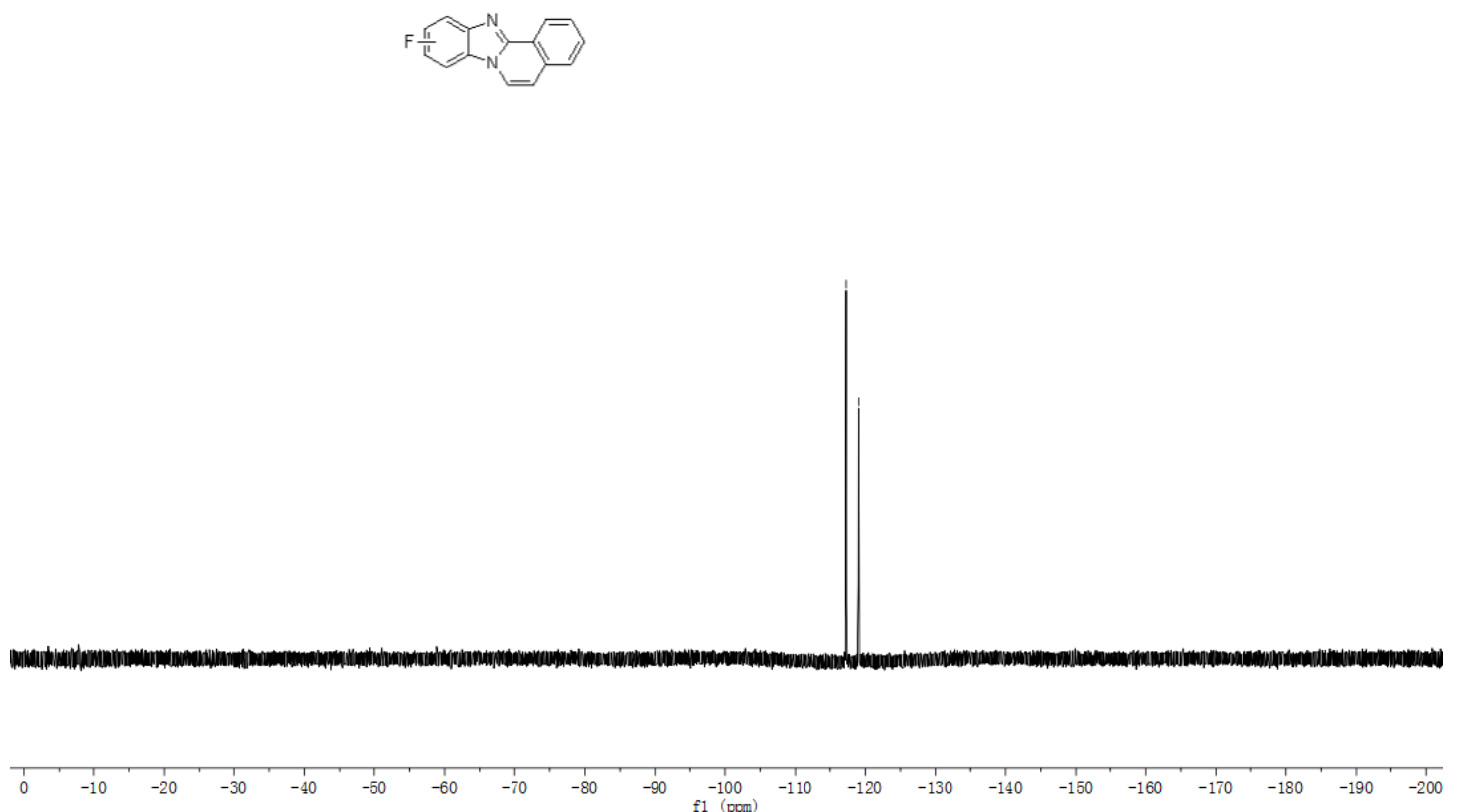

Fig S9. ${ }^{19} \mathrm{~F}$ NMR $\left(376 \mathrm{MHz}, \mathrm{CDCl}_{3}\right)$ of 10 -fluorobenzo[4,5]imidazo[2,1-a] isoquinoline and 9-fluorobenzo[4,5]imidazo[2,1-a]isoquinoline(2d)
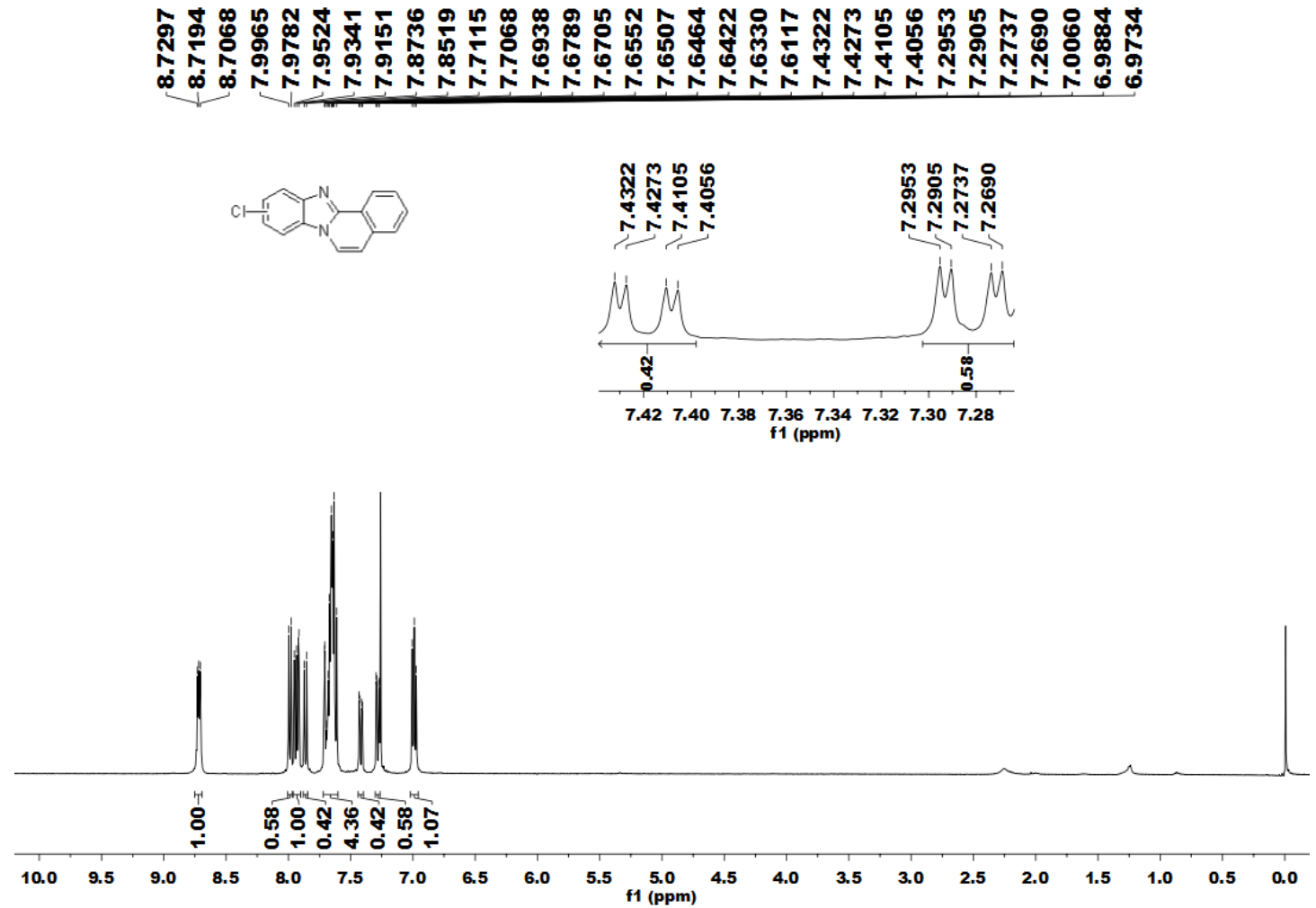

Fig S10. ${ }^{1} \mathrm{H}$ NMR (400 $\left.\mathrm{MHz}, \mathrm{CDCl}_{3}\right)$ of 10-Chlorobenzo[4,5]imidazo[2,1-a] isoquinoline and 9-chlorobenzo[4,5]imidazo[2,1-a]isoquinoline (2e) 


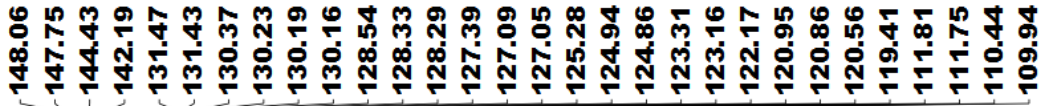
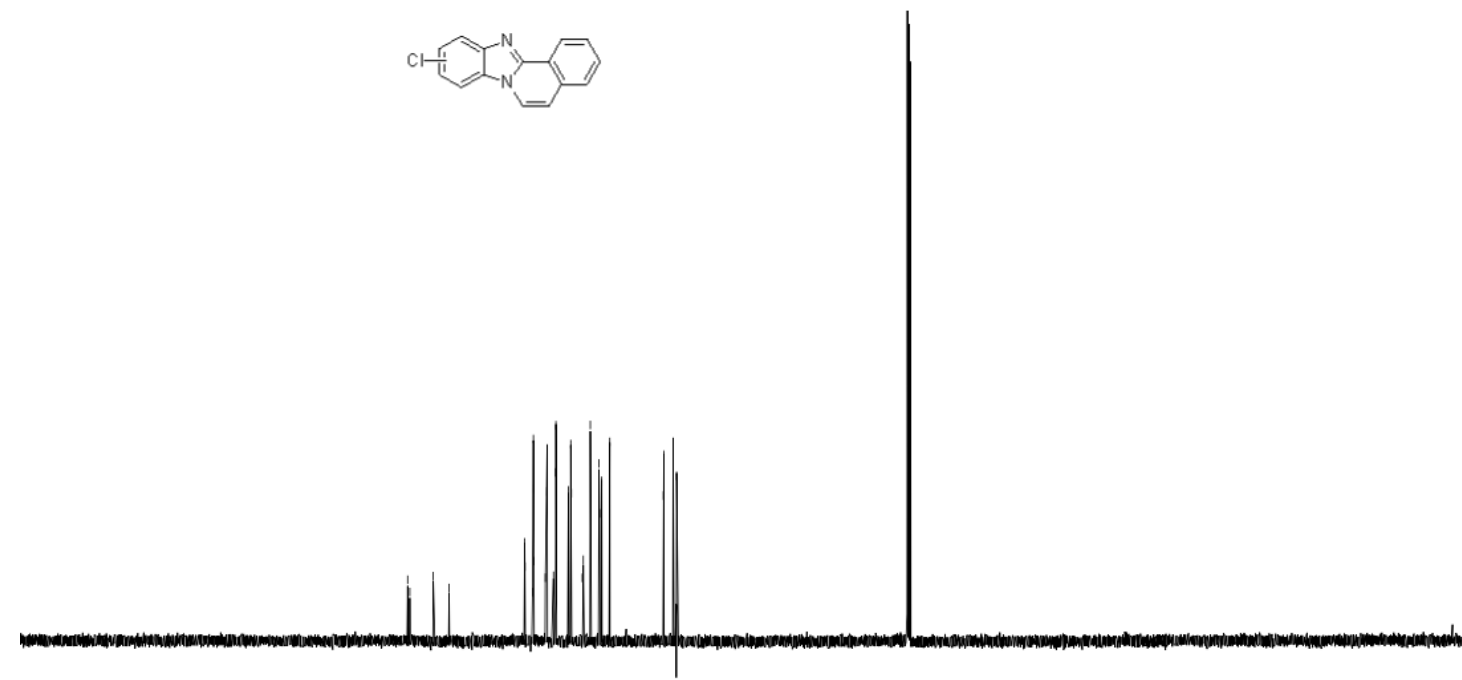

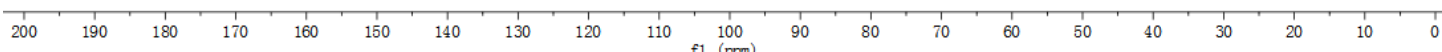

Fig S11. ${ }^{13} \mathrm{C}$ NMR $\left(150 \mathrm{MHz}, \mathrm{CDCl}_{3}\right)$ of 10-Chlorobenzo[4,5]imidazo[2,1-a] isoquinoline and 9-chlorobenzo[4,5]imidazo[2,1- $a$ ] isoquinoline (2e)
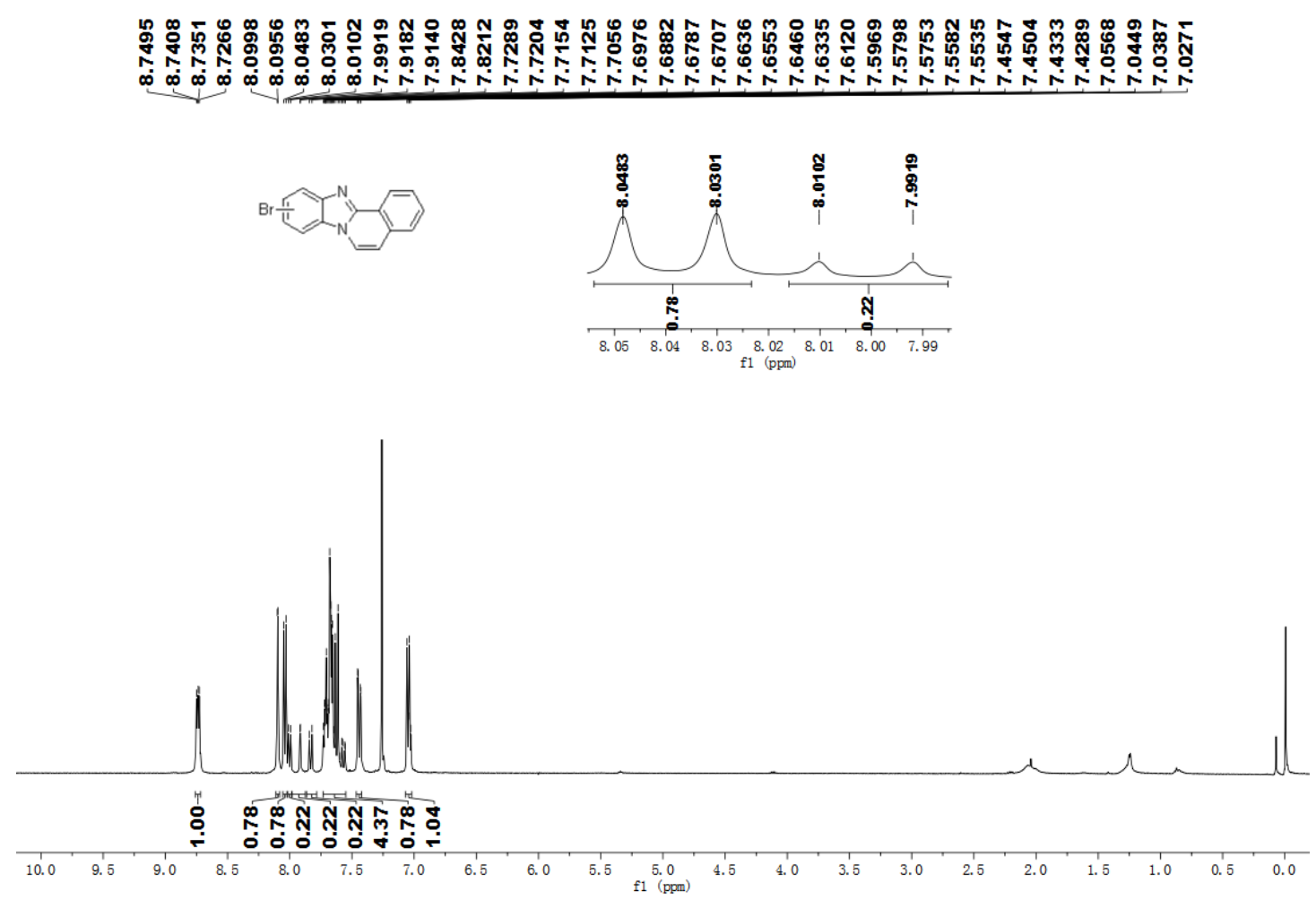

Fig S12. ${ }^{1} \mathrm{H}$ NMR $\left(400 \mathrm{MHz}, \mathrm{CDCl}_{3}\right)$ of 10-bromobenzo[4,5]imidazo[2,1-a] isoquinoline and 9-bromobenzo[4,5]imidazo[2,1-a]isoquinoline (2f) 


\section{มี้}
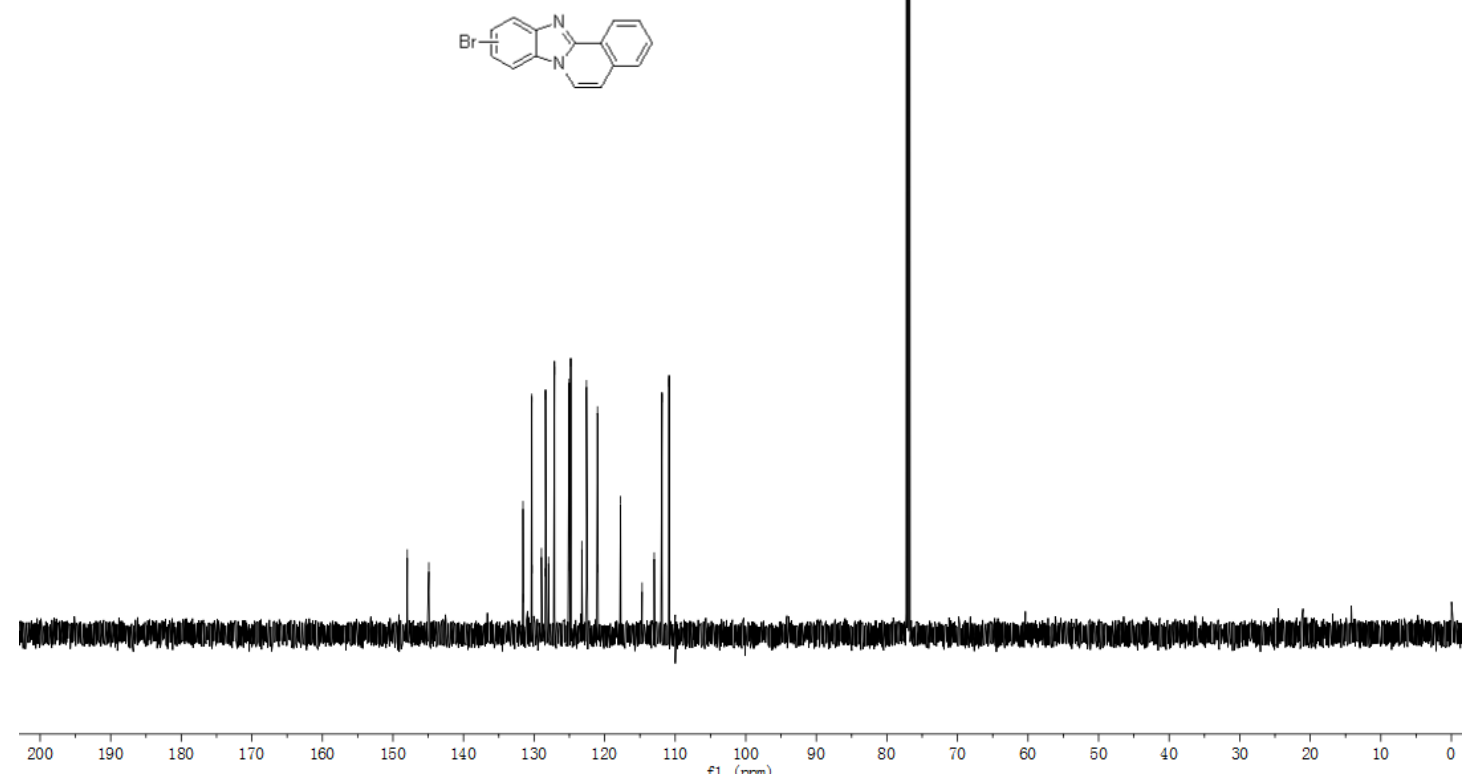

Fig S13. ${ }^{13} \mathrm{C}$ NMR $\left(150 \mathrm{MHz}, \mathrm{CDCl}_{3}\right)$ of 10-bromobenzo[4,5]imidazo[2,1-a] isoquinoline and 9-bromobenzo[4,5]imidazo[2,1- $a$ ] isoquinoline (2f)
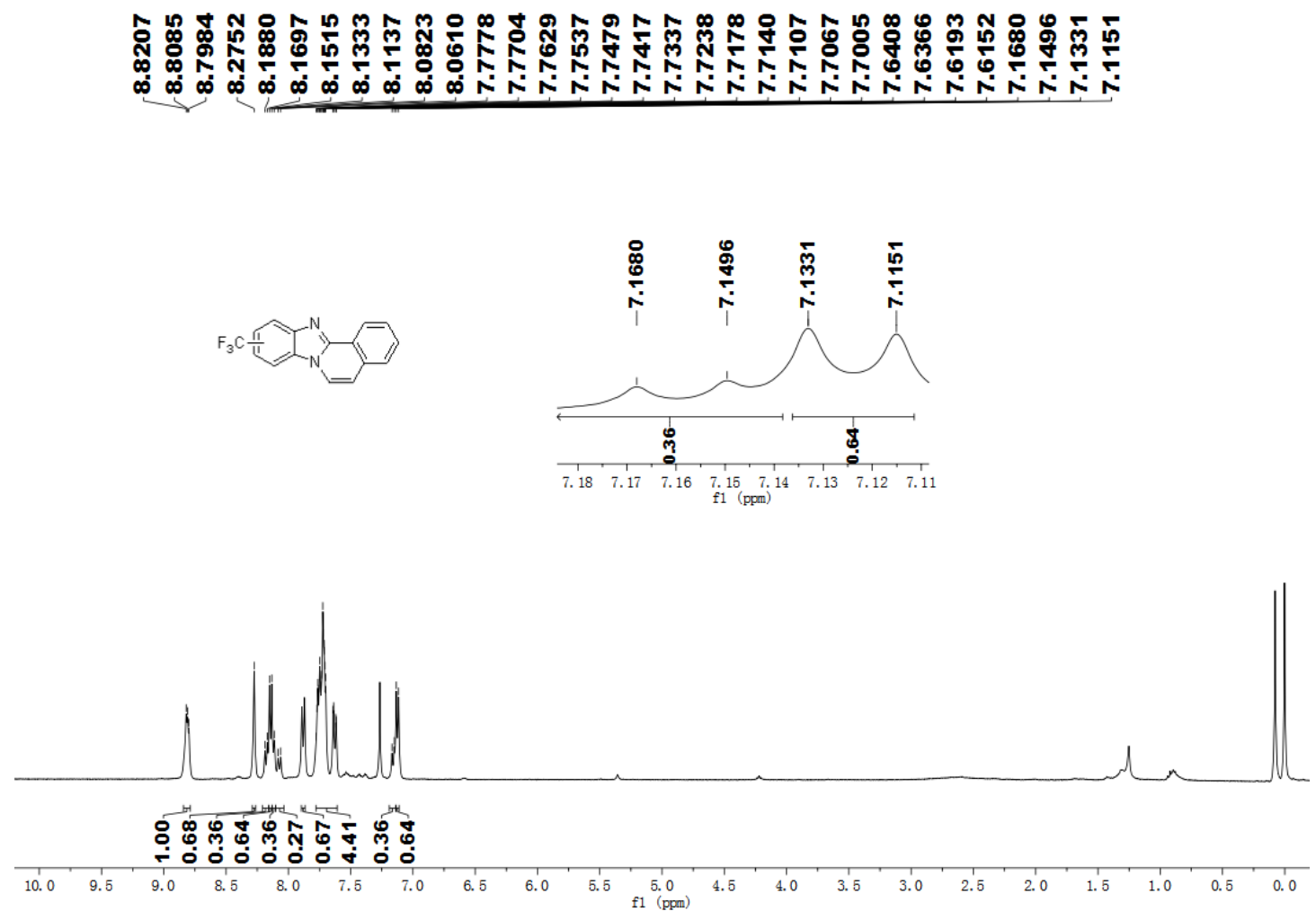

Fig S14. ${ }^{1} \mathrm{H}$ NMR $\left(400 \mathrm{MHz}, \mathrm{CDCl}_{3}\right.$ ) of 10-(trifluoromethyl)benzo[4,5]imidazo[2,1-a] isoquinoline and 9-(trifluoromethyl)benzo[4,5]imidazo[2,1-a]isoquinoline (2g) 


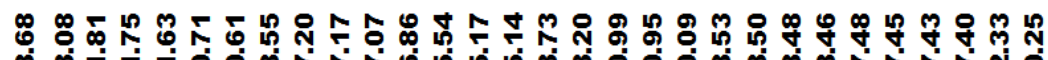

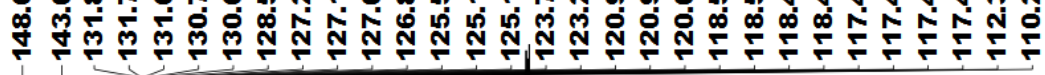
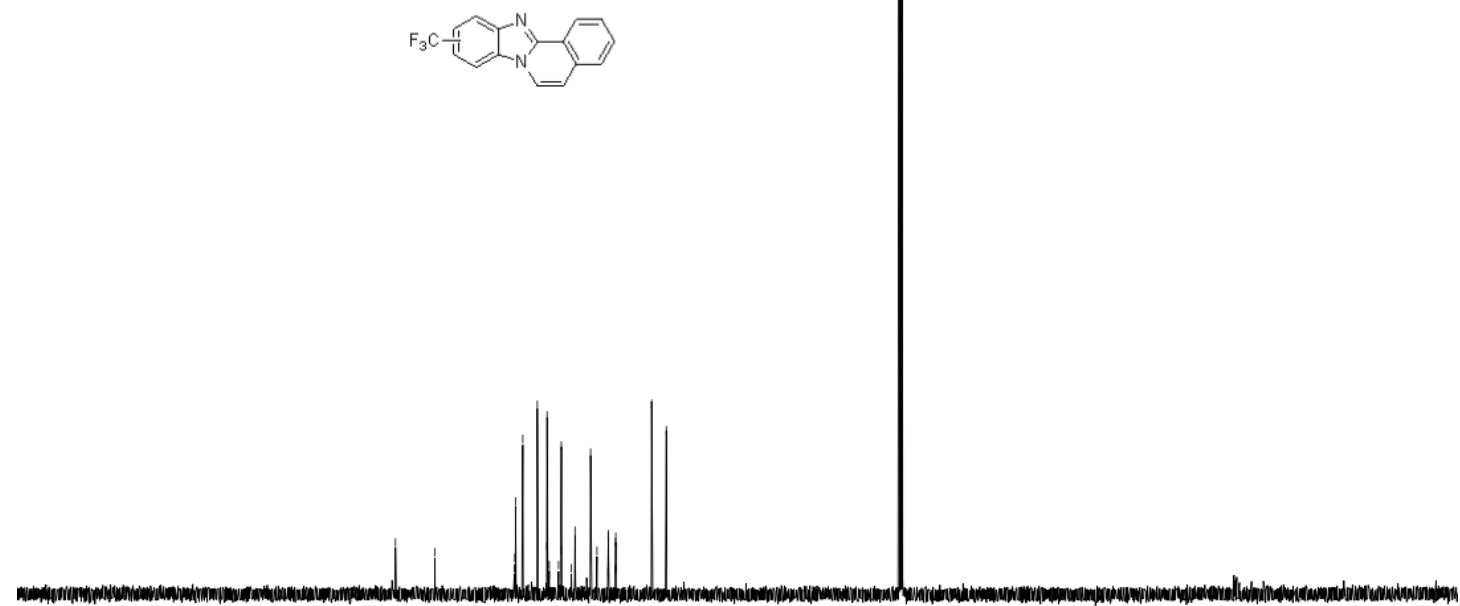

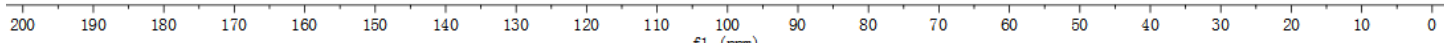

Fig S15. ${ }^{13} \mathrm{C} \mathrm{NMR}\left(150 \mathrm{MHz}, \mathrm{CDCl}_{3}\right)$ of 10-(trifluoromethyl)benzo[4,5]imidazo $[2,1-a]$ isoquinoline and 9-(trifluoromethyl)benzo[4,5]imidazo[2,1-a]isoquinoline (2g)

管
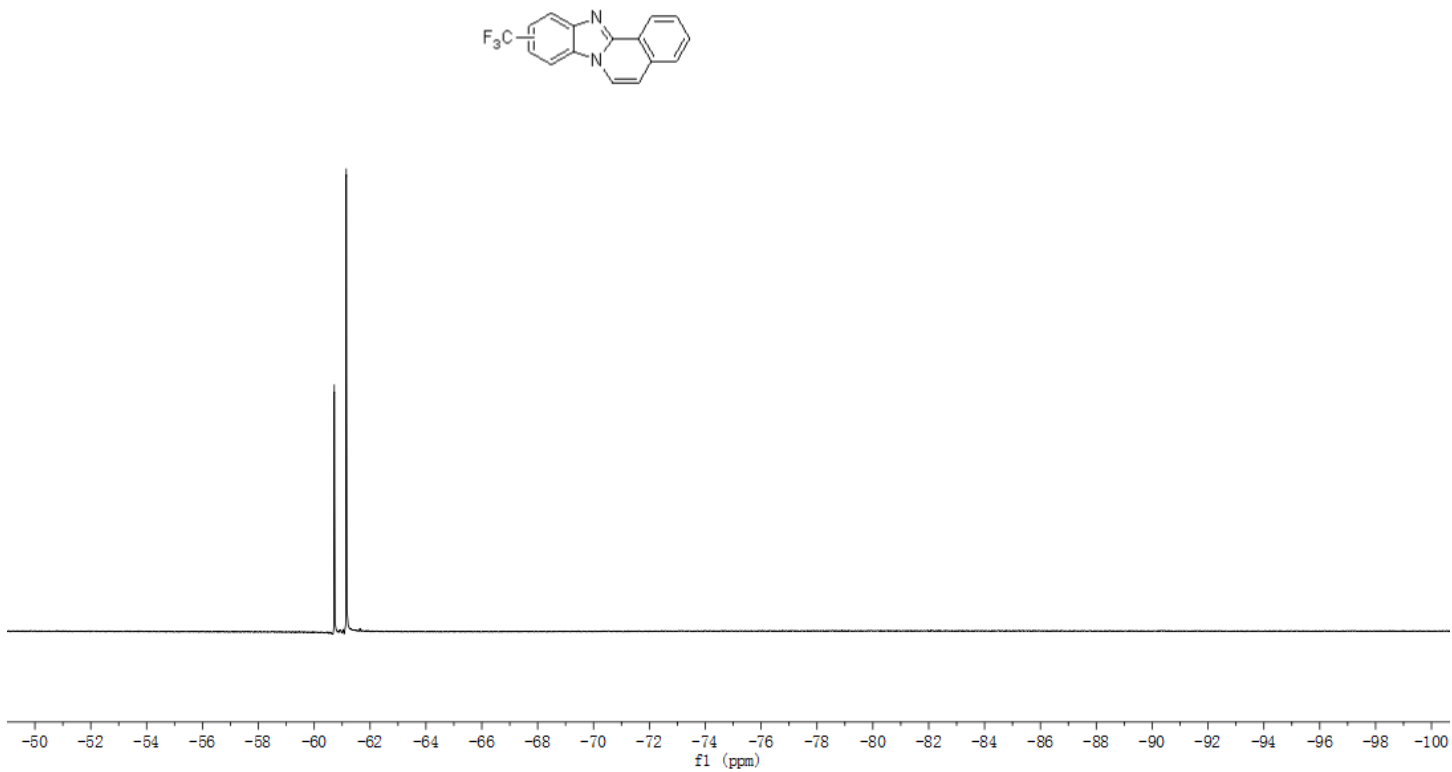

Fig S16. ${ }^{19} \mathrm{~F}$ NMR (376 $\mathrm{MHz}, \mathrm{CDCl}_{3}$ ) of 10-(trifluoromethyl)benzo[4,5]imidazo $[2,1-a]$ isoquinoline and 9-(trifluoromethyl)benzo[4,5]imidazo[2,1-a]isoquinoline (2g) 

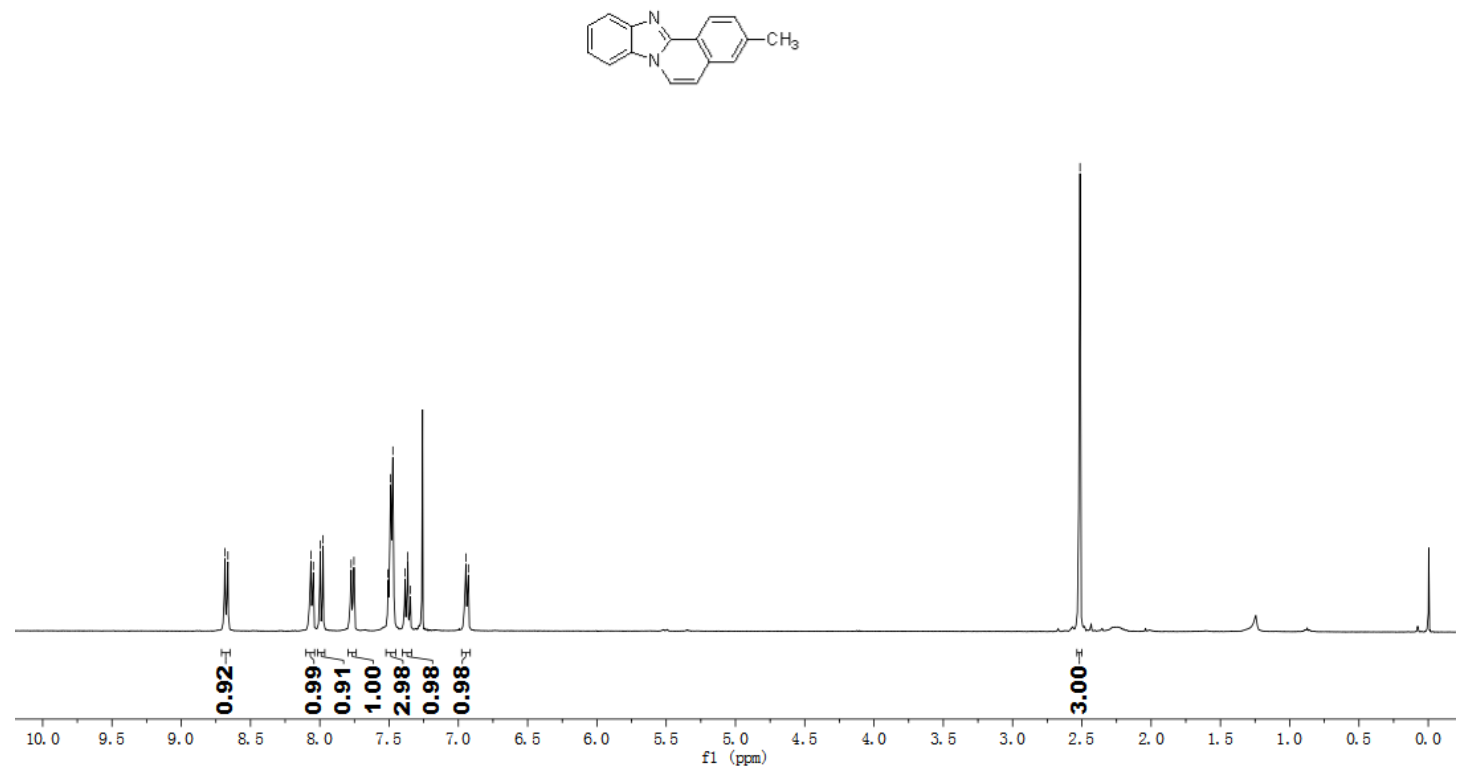

Fig S17. ${ }^{1} \mathrm{H}$ NMR (400 $\left.\mathrm{MHz}, \mathrm{CDCl}_{3}\right)$ of 3-methylbenzo[4,5]imidazo[2,1-a] isoquinoline (2h)

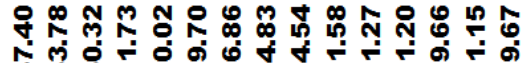

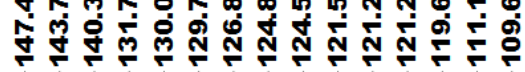

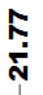
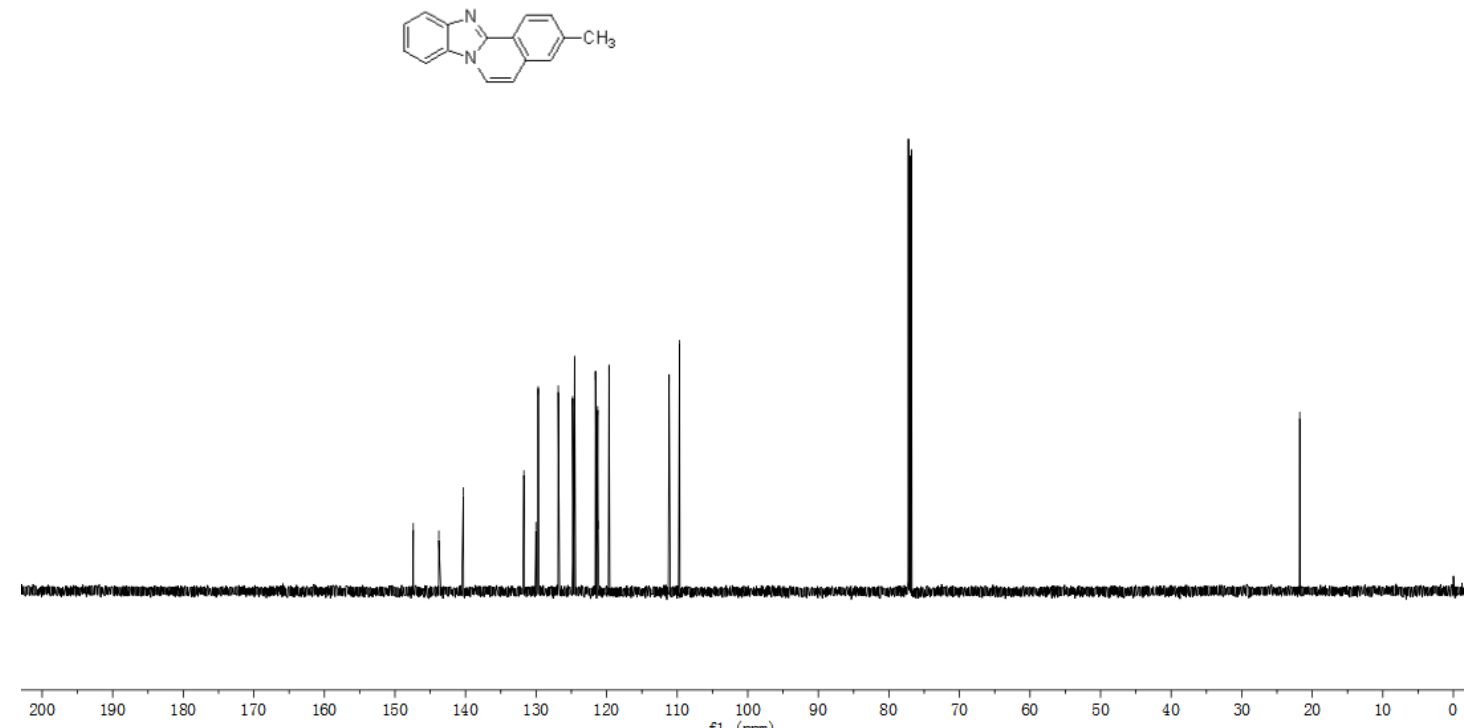

Fig S18. ${ }^{13} \mathrm{C}$ NMR $\left(150 \mathrm{MHz}, \mathrm{CDCl}_{3}\right)$ of 3-methylbenzo[4,5]imidazo[2,1-a] isoquinoline (2h) 


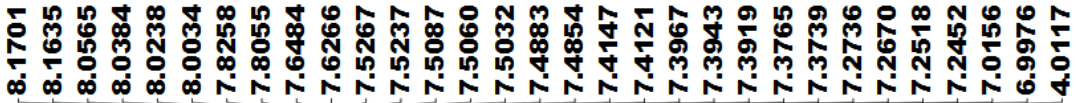

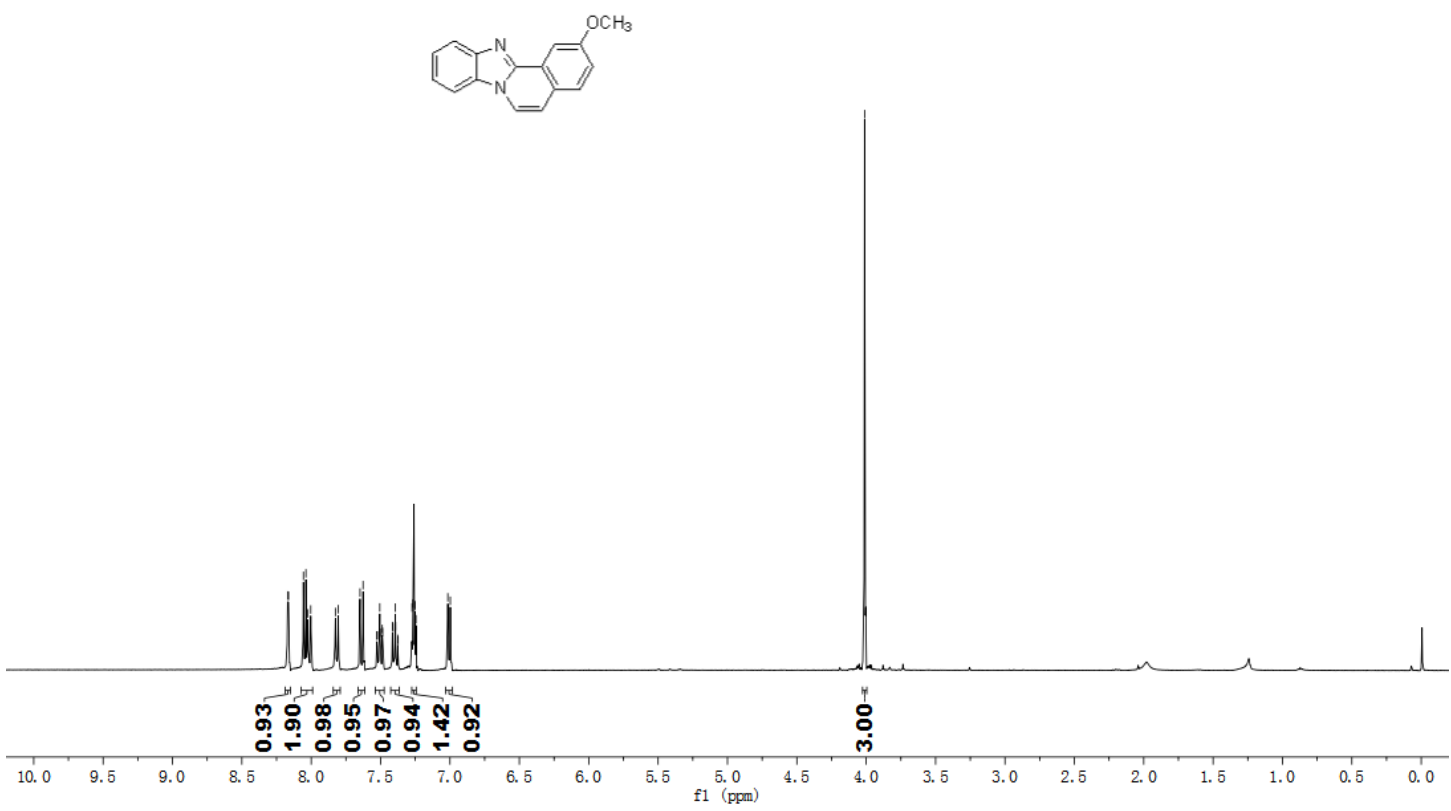

Fig S19. ${ }^{1} \mathrm{H}$ NMR (400 $\left.\mathrm{MHz}, \mathrm{CDCl}_{3}\right)$ of 2-methoxybenzo[4,5]imidazo[2,1-a] isoquinoline $(\mathbf{2 i})$

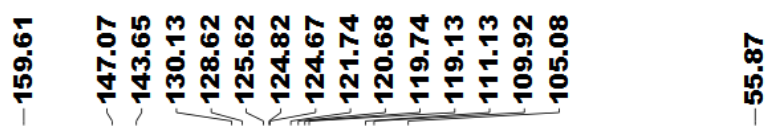

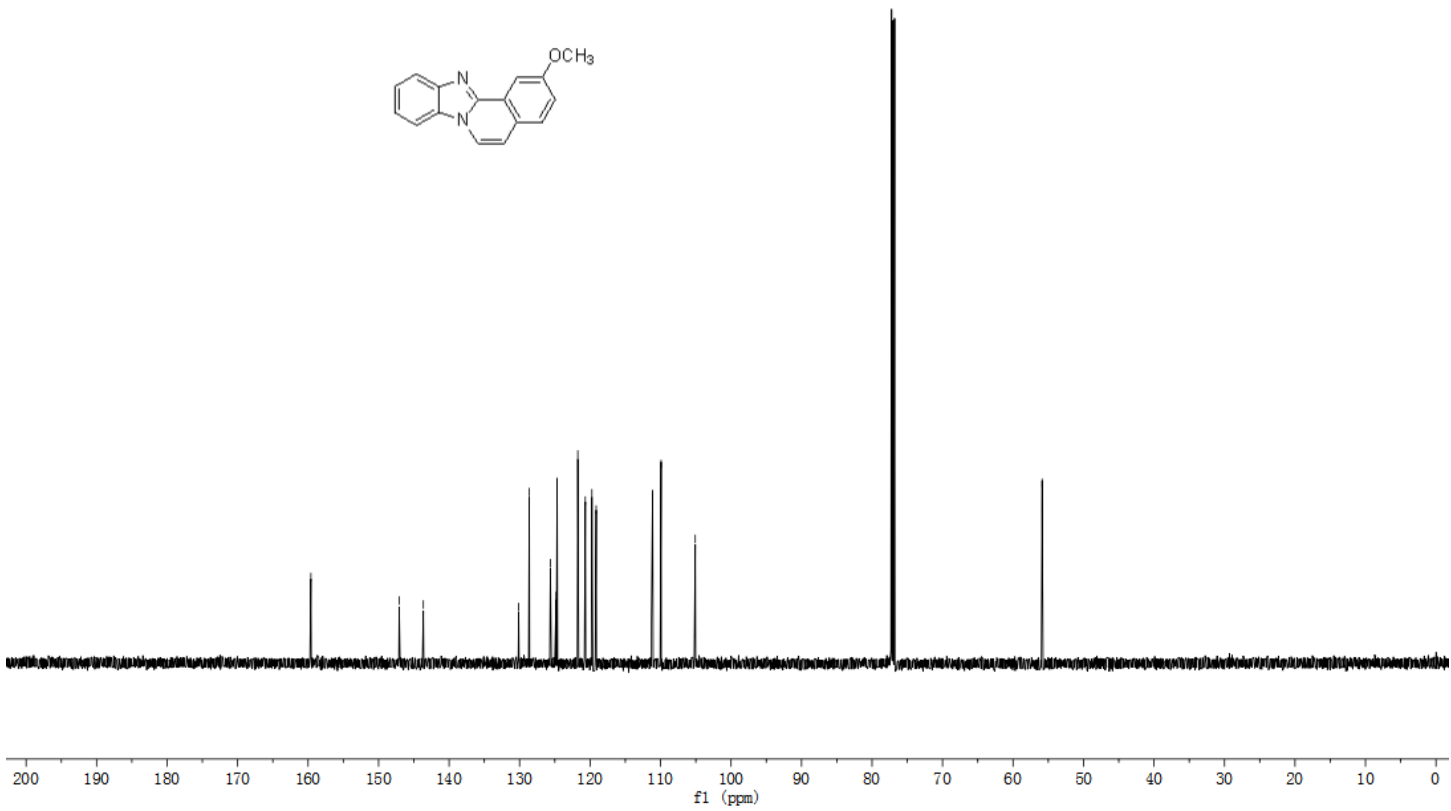

Fig S20. ${ }^{13} \mathrm{C}$ NMR $\left(150 \mathrm{MHz}, \mathrm{CDCl}_{3}\right)$ of 2-methoxybenzo[4,5]imidazo[2,1-a] isoquinoline (2i) 


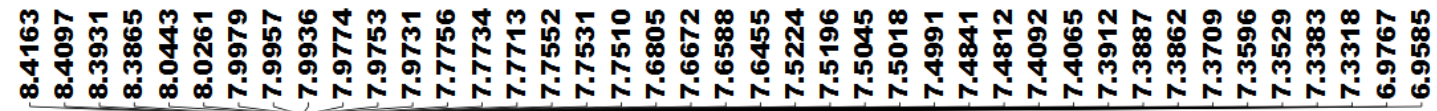

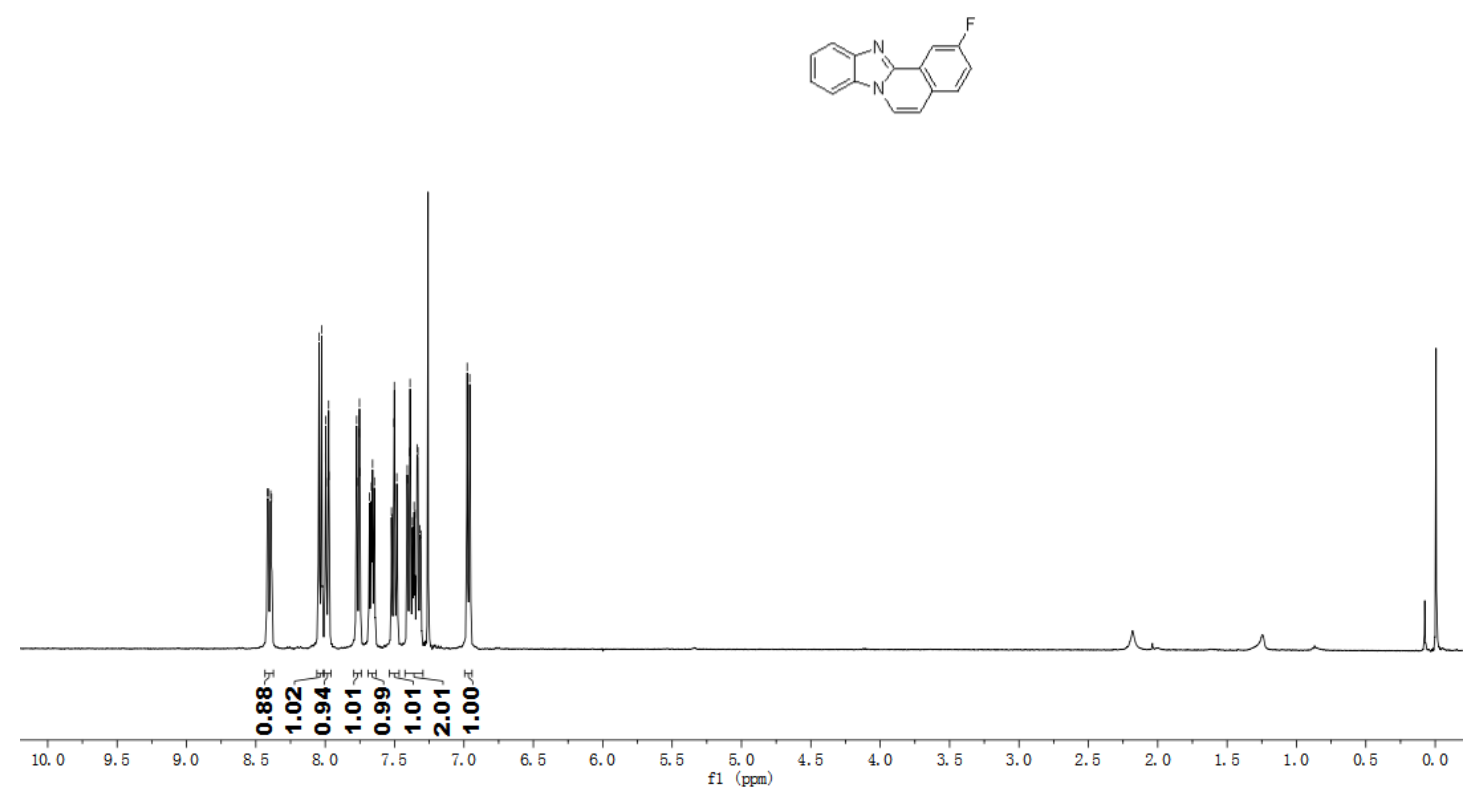

Fig S21. ${ }^{1} \mathrm{H}$ NMR $\left(400 \mathrm{MHz}, \mathrm{CDCl}_{3}\right)$ of 2-fluorobenzo[4,5]imidazo[2,1-a] isoquinoline $(\mathbf{2} \mathbf{j})$
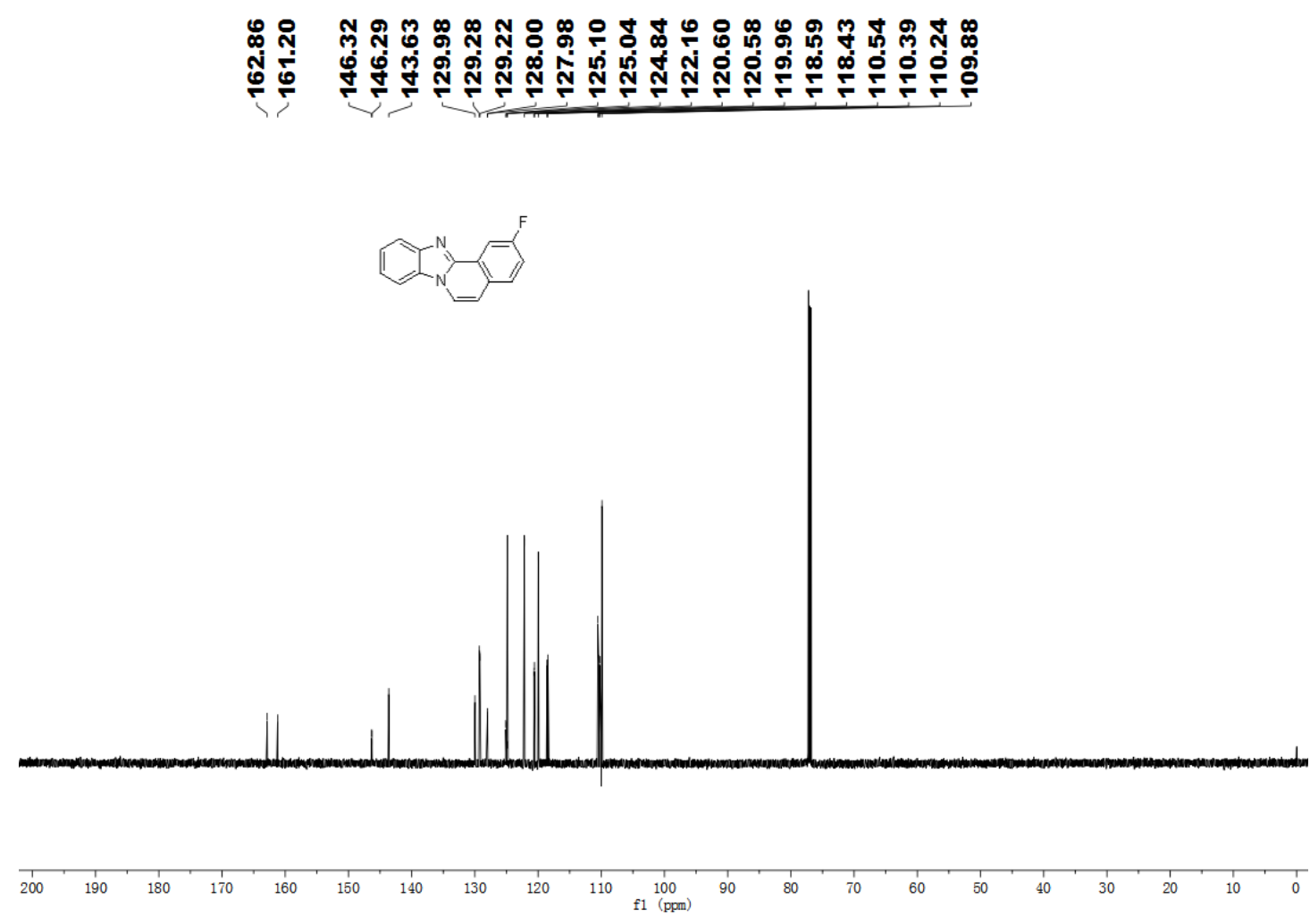

Fig S22. ${ }^{13} \mathrm{C}$ NMR (150 $\left.\mathrm{MHz}, \mathrm{CDCl}_{3}\right)$ of 2-fluorobenzo[4,5]imidazo[2,1-a] isoquinoline (2j) 


\section{$\stackrel{n}{1 ! n}$}
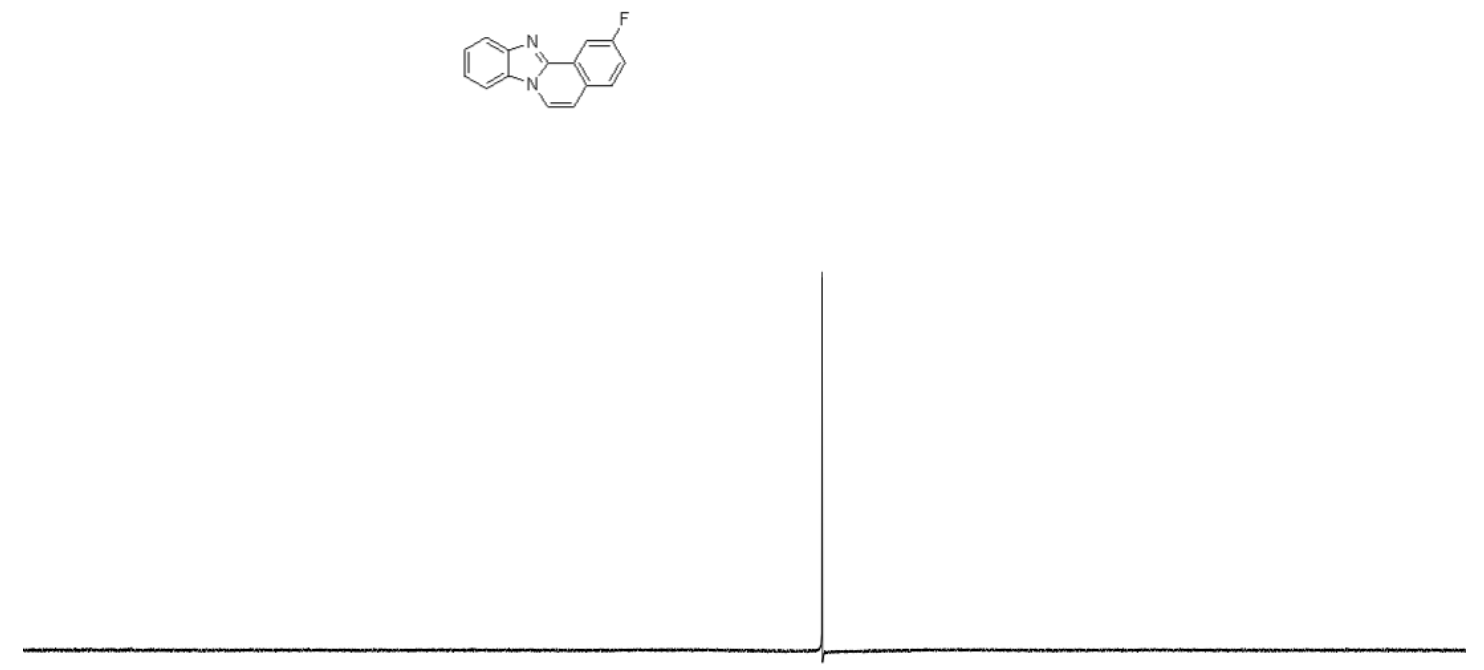

Fig S23. ${ }^{19} \mathrm{~F}$ NMR (376 $\left.\mathrm{MHz}, \mathrm{CDCl}_{3}\right)$ of 2-fluorobenzo[4,5]imidazo[2,1-a] isoquinoline (2⿺j)

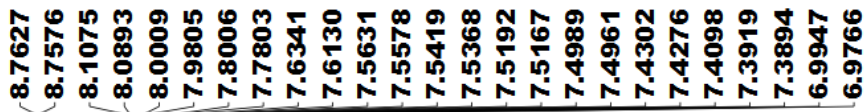
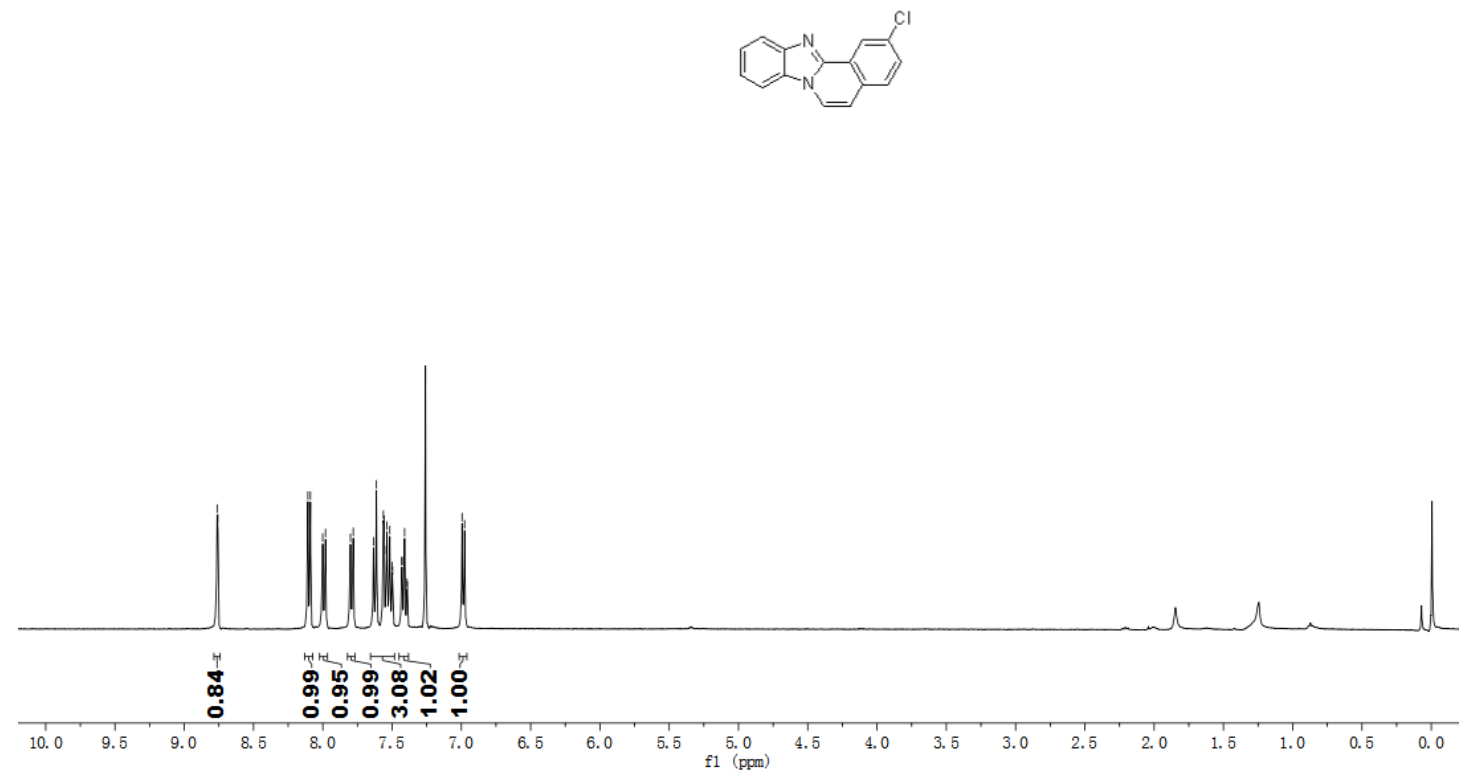

Fig S24. ${ }^{1} \mathrm{H}$ NMR (400 $\left.\mathrm{MHz}, \mathrm{CDCl}_{3}\right)$ of 2-Chlorobenzo[4,5]imidazo[2,1-a] isoquinoline (2k) 

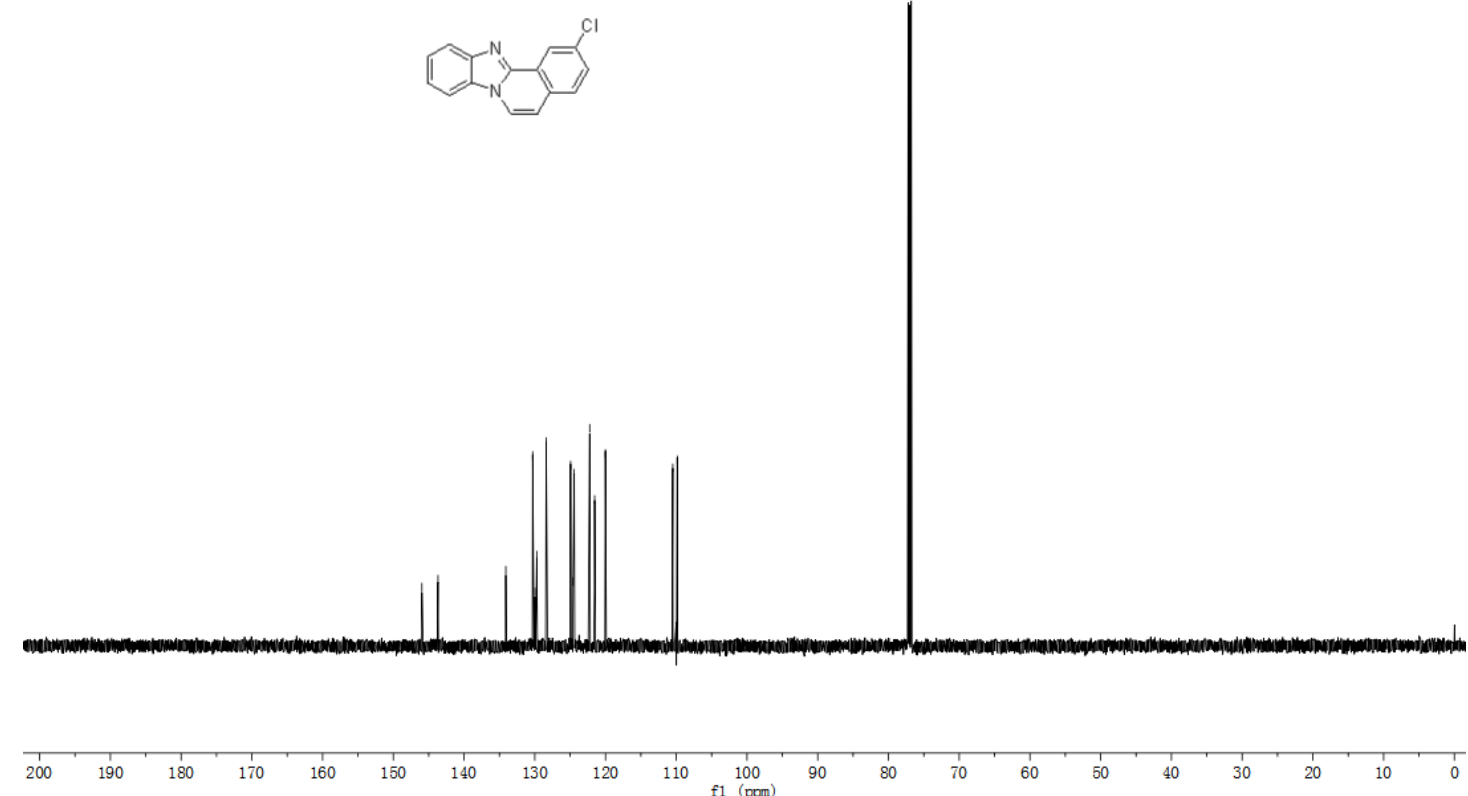

Fig S25. ${ }^{13} \mathrm{C}$ NMR $\left(150 \mathrm{MHz}, \mathrm{CDCl}_{3}\right)$ of 2-Chlorobenzo[4,5]imidazo[2,1-a] isoquinoline $(\mathbf{2 k})$
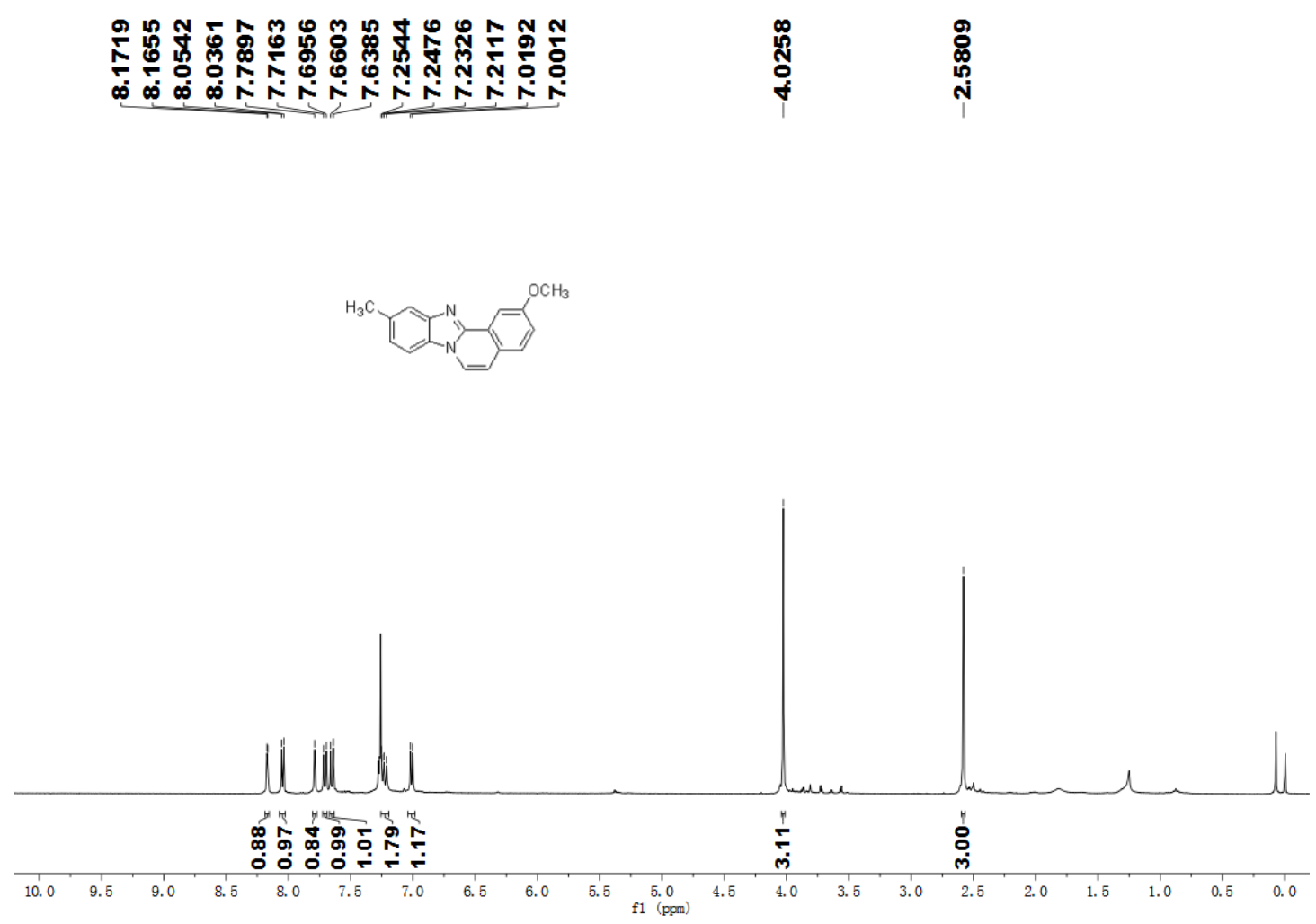

Fig S26. ${ }^{1} \mathrm{H}$ NMR $\left(400 \mathrm{MHz}, \mathrm{CDCl}_{3}\right)$ of 2-methoxy-10-methylbenzo[4,5]imidazo $[2,1-a]$ isoquinoline (2l) 

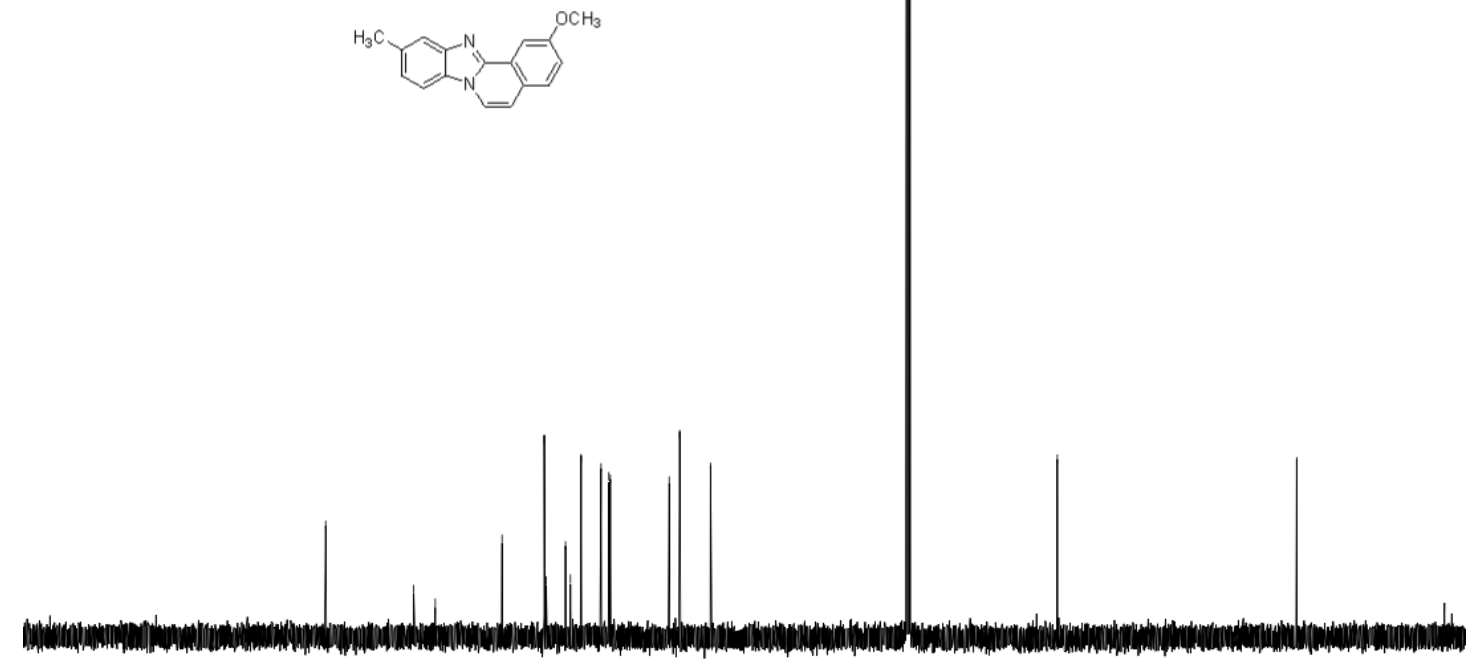

$200 \quad 190 \quad 180 \quad 170 \quad 180$

Fig S27. ${ }^{13} \mathrm{C}$ NMR $\left(150 \mathrm{MHz}, \mathrm{CDCl}_{3}\right)$ of 2-methoxy-10-methylbenzo[4,5]imidazo [2,1- $a]$ isoquinoline (2l)
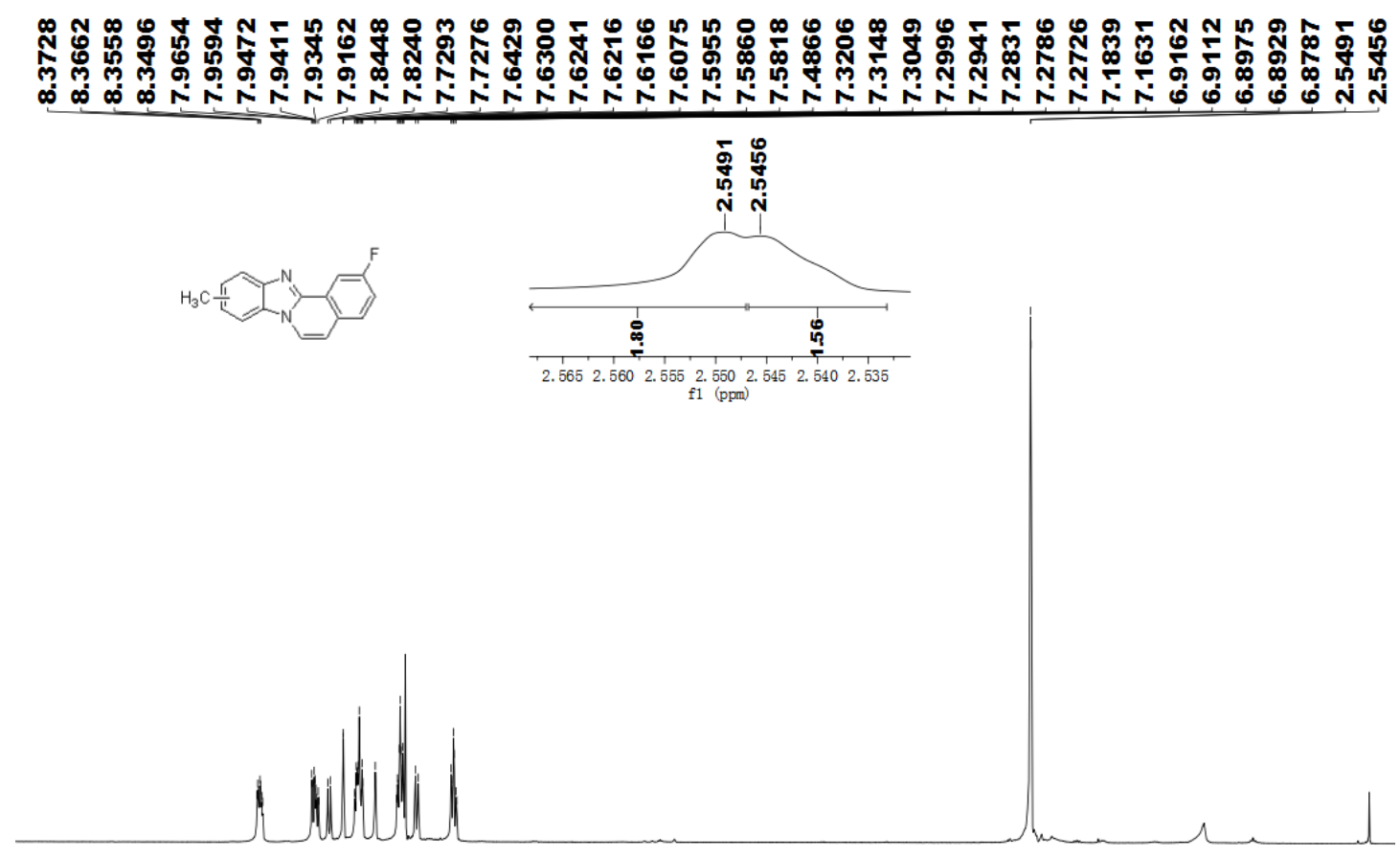

กั

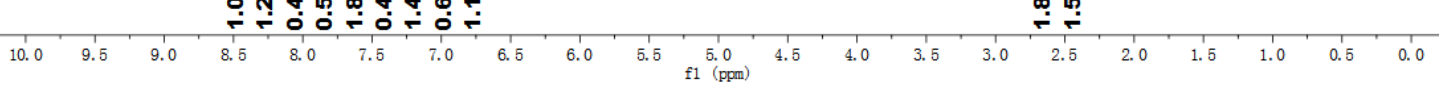

Fig S28. ${ }^{1} \mathrm{H}$ NMR (400 $\left.\mathrm{MHz}, \mathrm{CDCl}_{3}\right)$ of 2-fluoro-10-methylbenzo[4,5]imidazo [2,1-a]isoquinoline and 2-fluoro-9-methylbenzo[4,5]imidazo[2,1-a]isoquinoline (2m) 

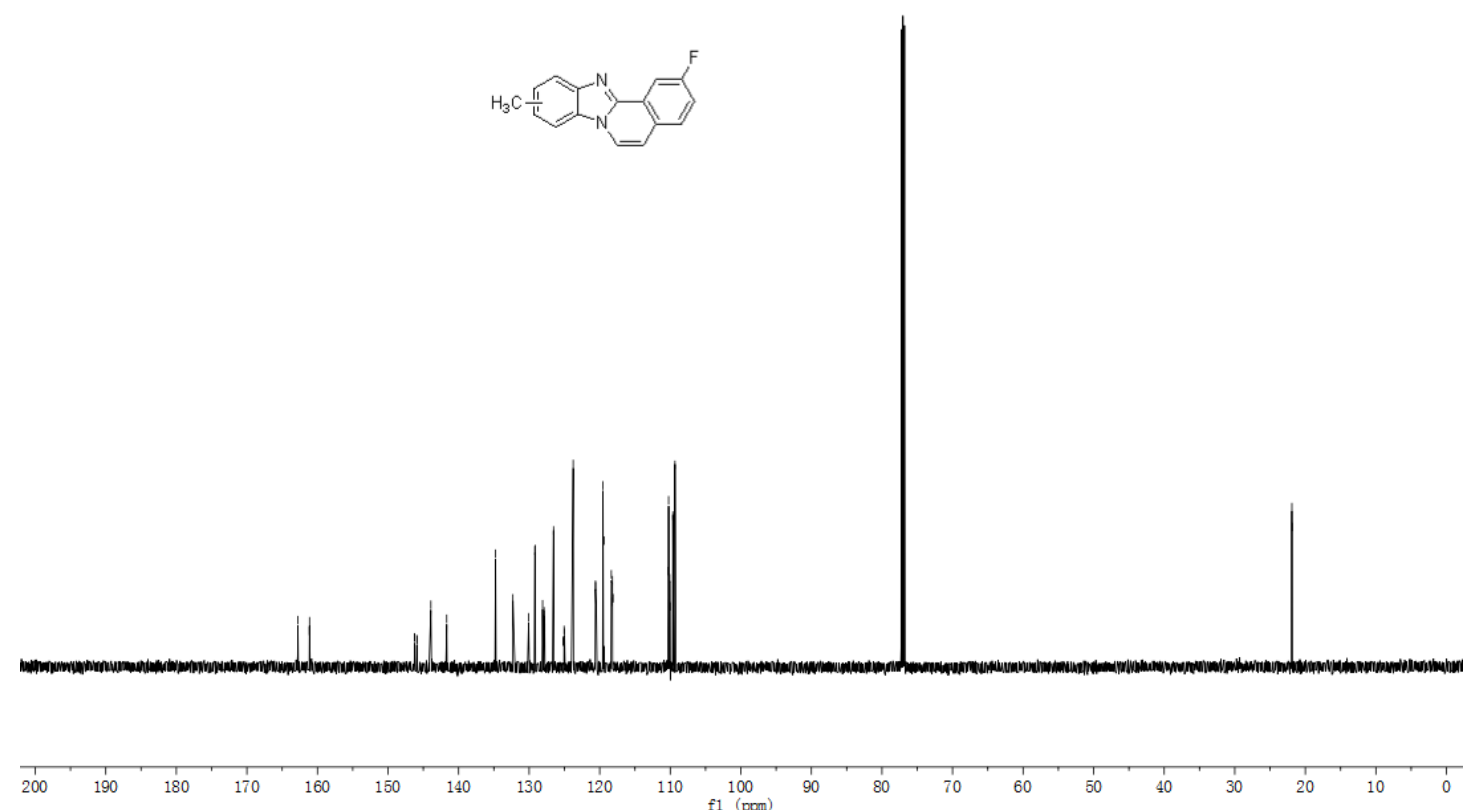

Fig S29. ${ }^{13} \mathrm{C}$ NMR (150 MHz, $\left.\mathrm{CDCl}_{3}\right)$ of 2-fluoro-10-methylbenzo[4,5]imidazo $[2,1-a]$ isoquinoline and 2-fluoro-9-methylbenzo[4,5]imidazo[2,1-a]isoquinoline (2m)

$\underset{i}{\stackrel{i}{i}}$
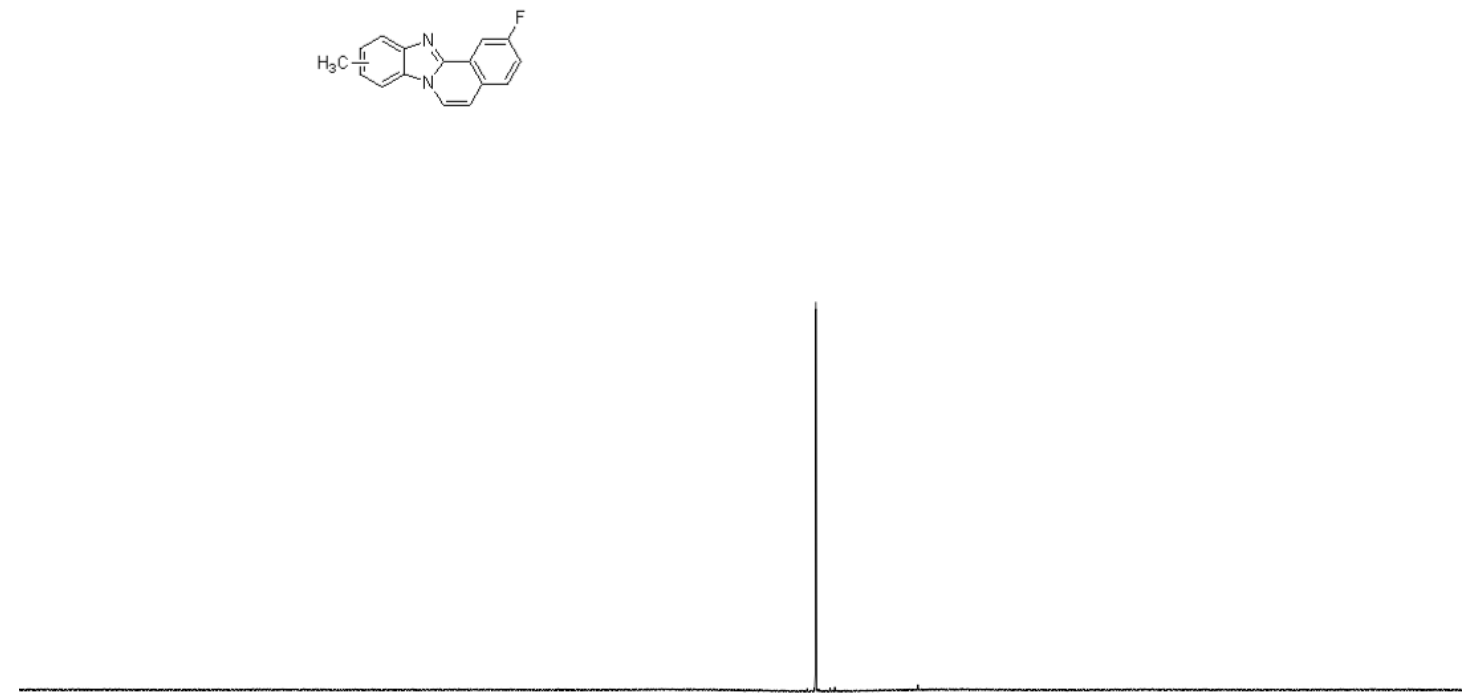

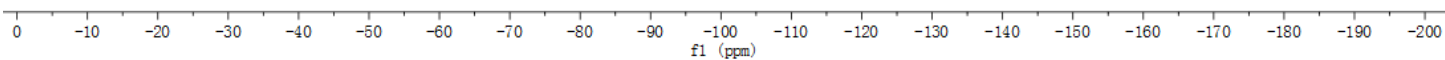

Fig S30. ${ }^{19} \mathrm{~F}$ NMR (376 $\left.\mathrm{MHz}, \mathrm{CDCl}_{3}\right)$ of 2-fluoro-10-methylbenzo[4,5]imidazo [2,1-a]isoquinoline and 2-fluoro-9-methylbenzo[4,5]imidazo[2,1-a]isoquinoline (2m) 

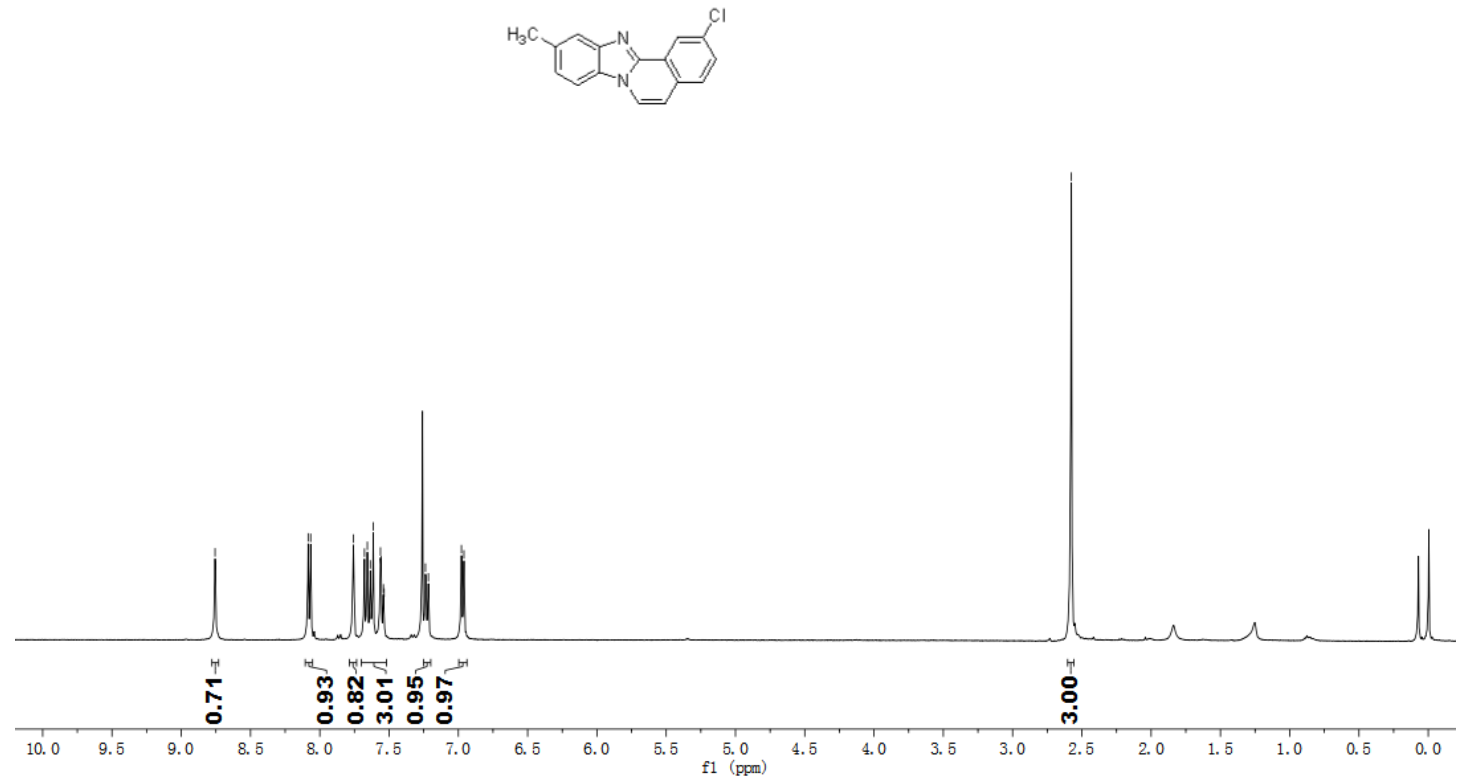

Fig S31. ${ }^{1} \mathrm{H}$ NMR $\left(400 \mathrm{MHz}, \mathrm{CDCl}_{3}\right)$ of 2-chloro-10-methylbenzo[4,5]imidazo $[2,1-a]$ isoquinoline $(\mathbf{2 n})$

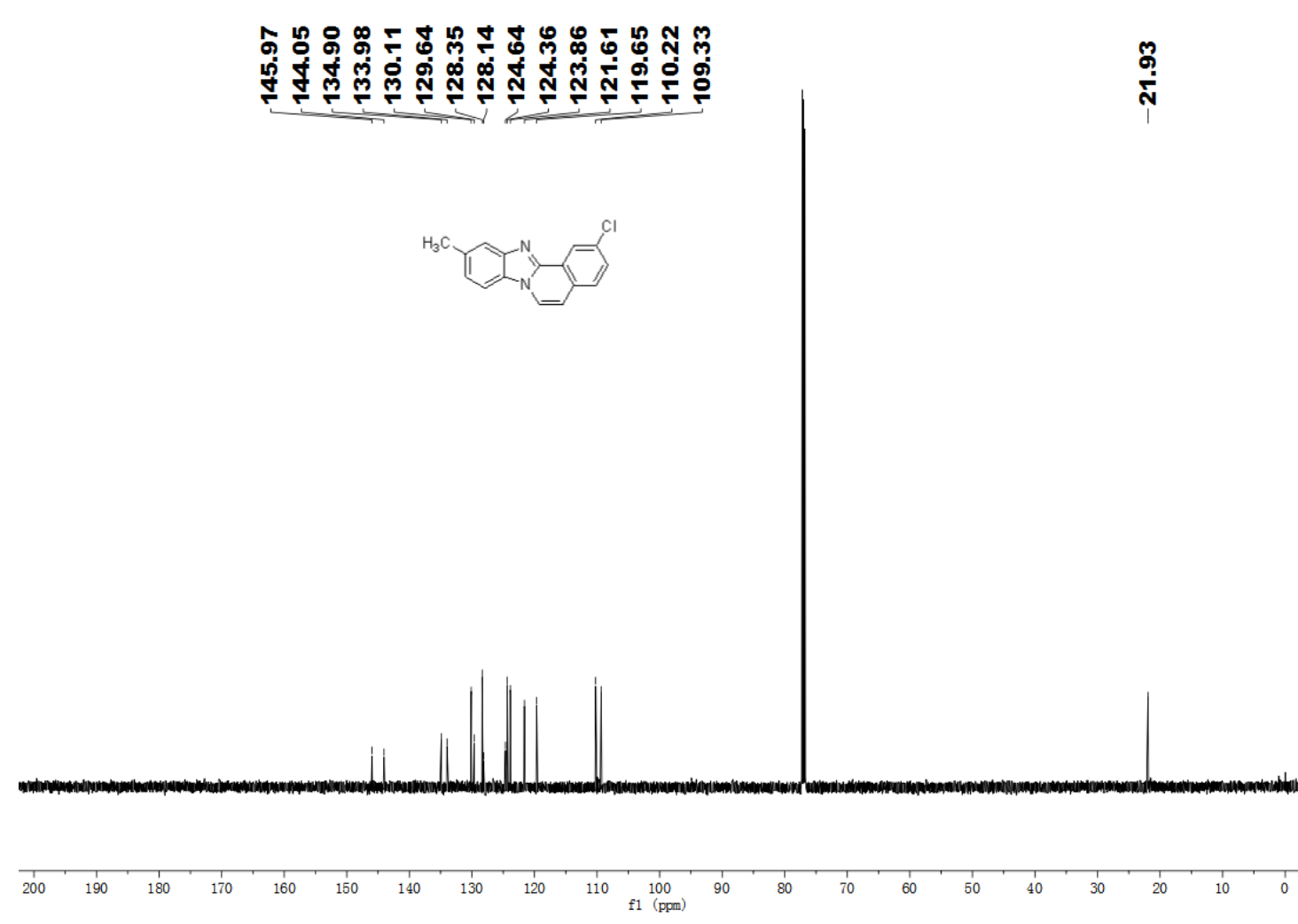

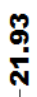

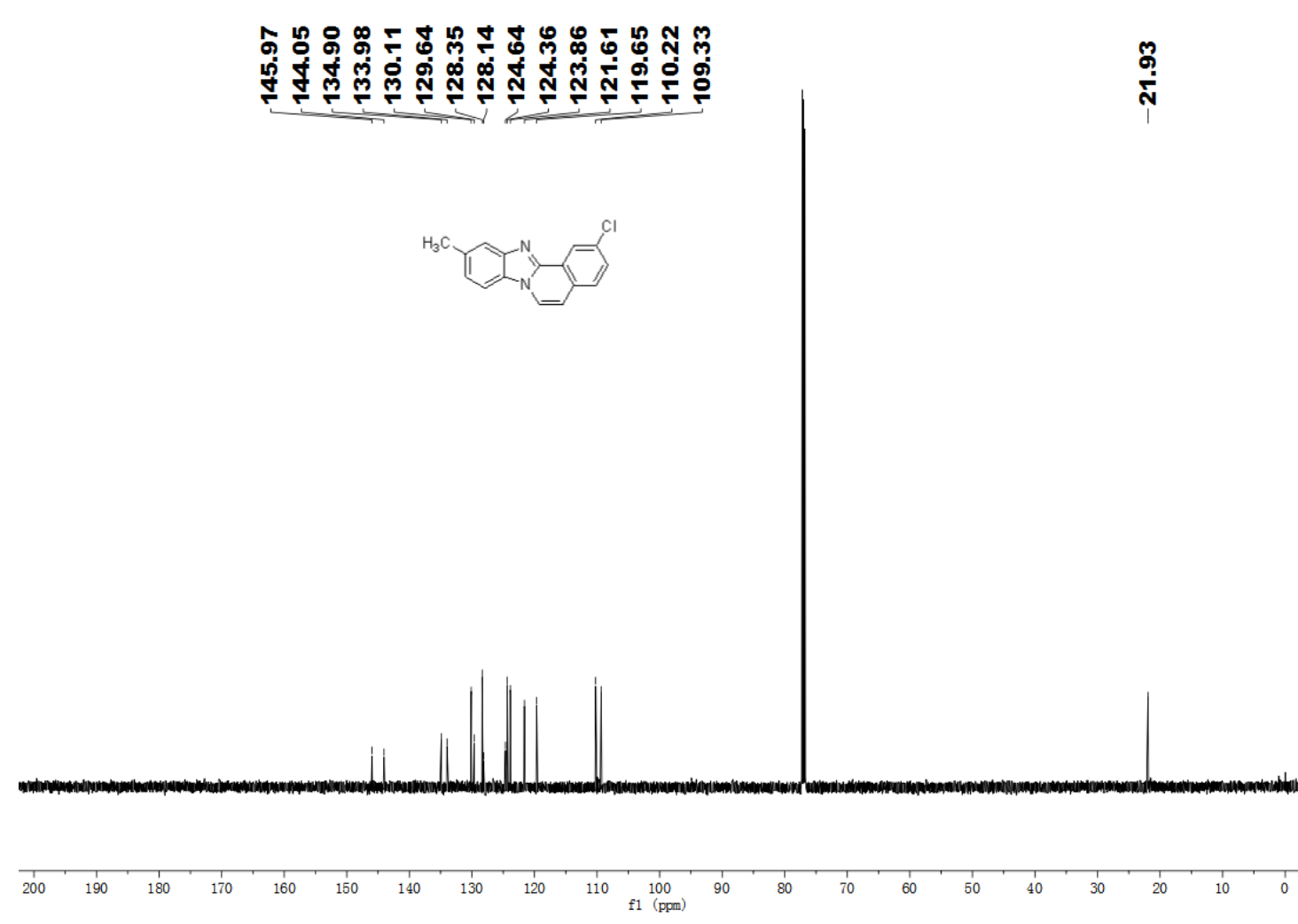

Fig S32. ${ }^{13} \mathrm{C}$ NMR $\left(150 \mathrm{MHz}, \mathrm{CDCl}_{3}\right)$ of 2-chloro-10-methylbenzo[4,5]imidazo $[2,1-a]$ isoquinoline (2n) 

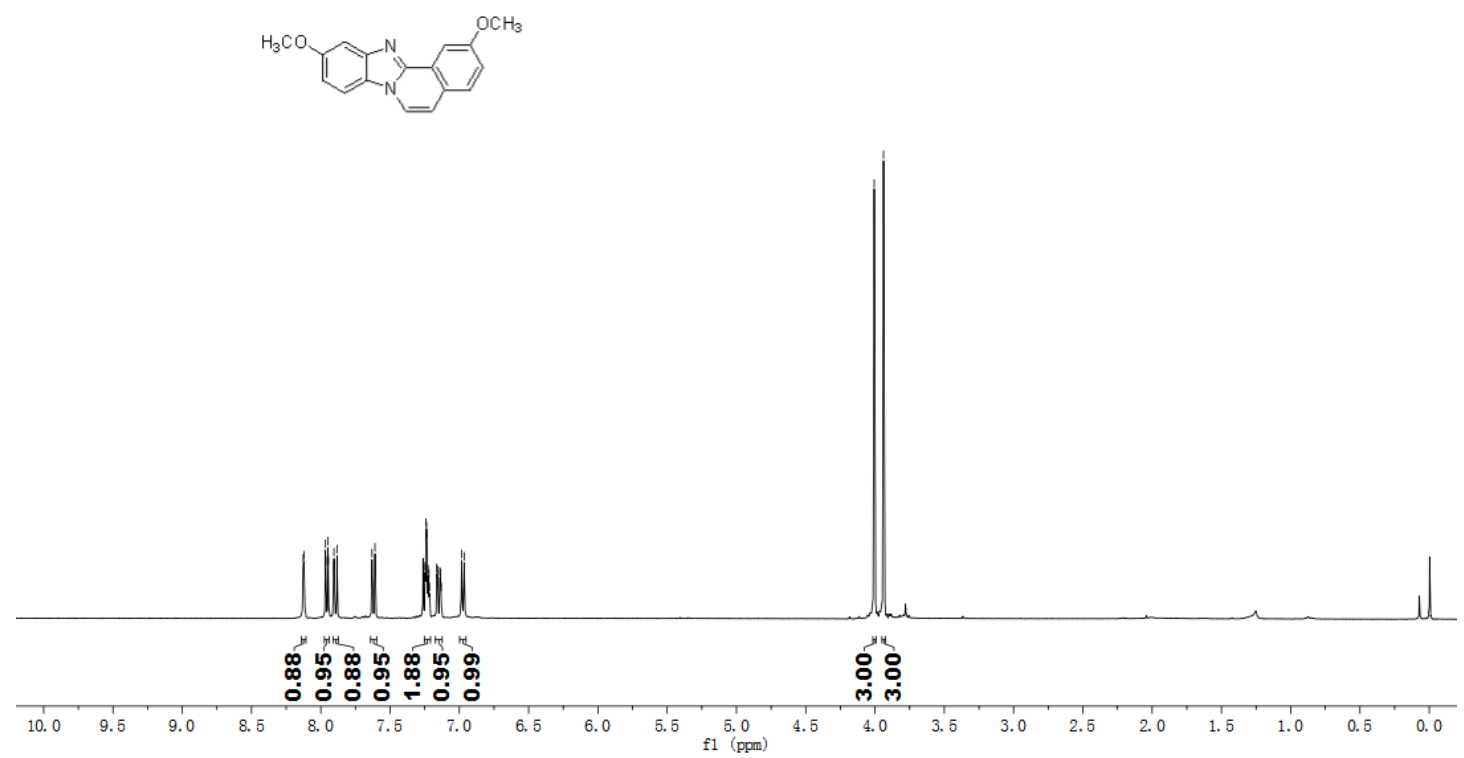

Fig S33. ${ }^{1} \mathrm{H}$ NMR (400 $\left.\mathrm{MHz}, \mathrm{CDCl}_{3}\right)$ of 2,10-dimethoxybenzo[4,5]imidazo $[2,1-a]$ isoquinoline $(\mathbf{2 o})$

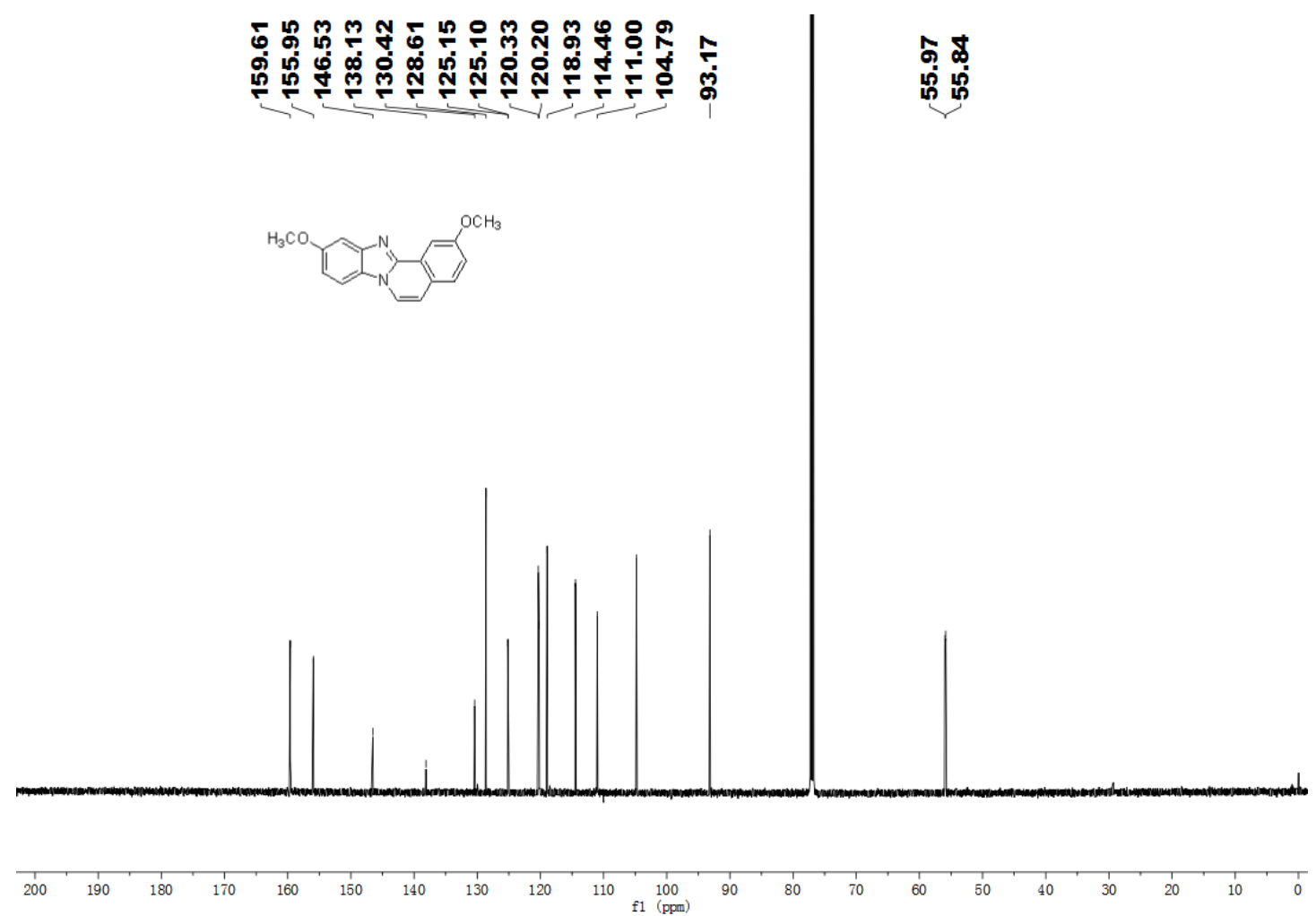

Fig S34. ${ }^{13} \mathrm{C}$ NMR $\left(150 \mathrm{MHz}, \mathrm{CDCl}_{3}\right)$ of 2,10-dimethoxybenzo[4,5]imidazo $[2,1-a]$ isoquinoline $(\mathbf{2 o})$ 

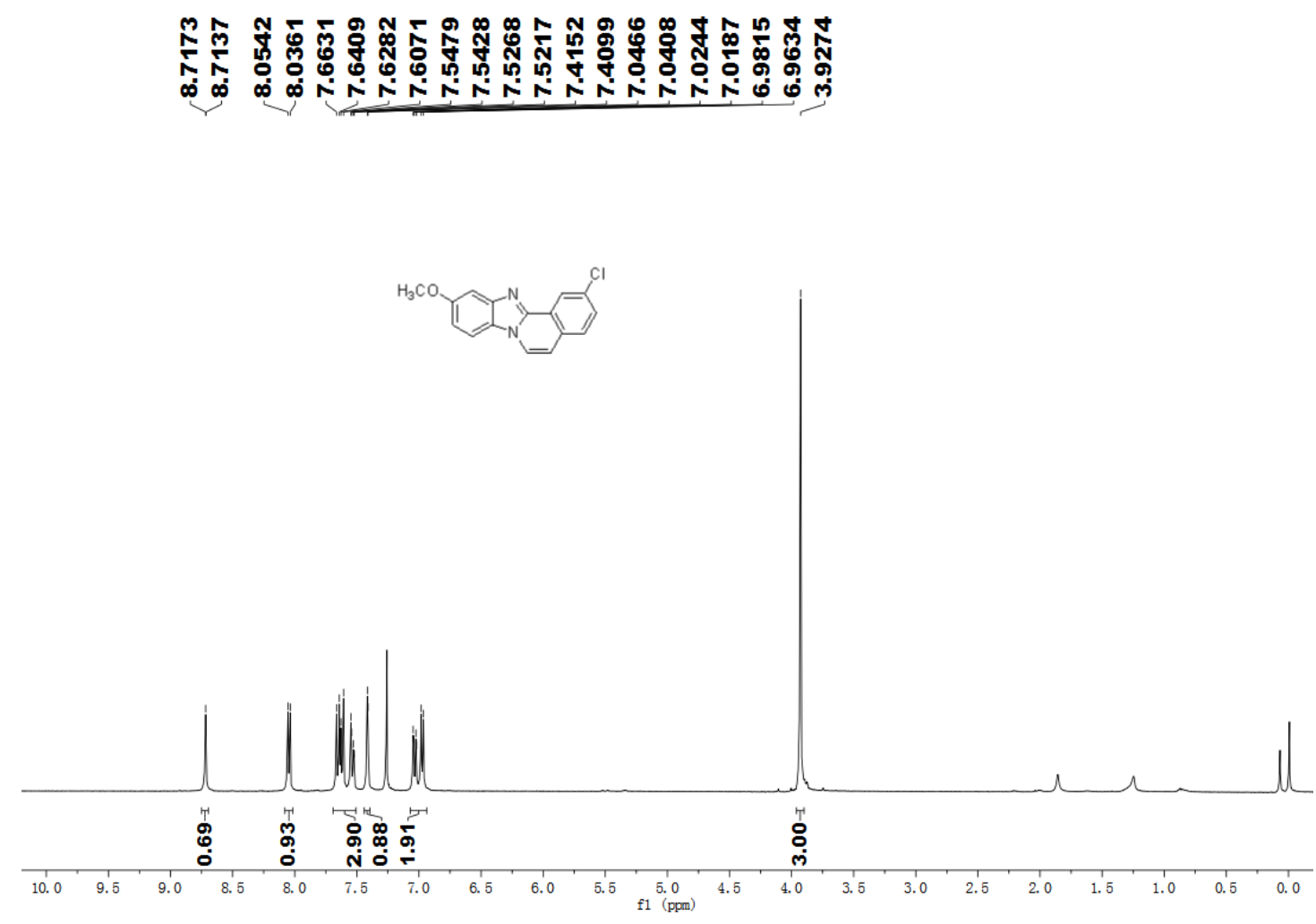

Fig S35. ${ }^{1} \mathrm{H}$ NMR (400 $\mathrm{MHz}, \mathrm{CDCl}_{3}$ ) of 2-chloro-10-methoxybenzo[4,5]imidazo $[2,1-a]$ isoquinoline (2p)

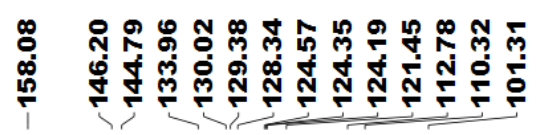

กิ่
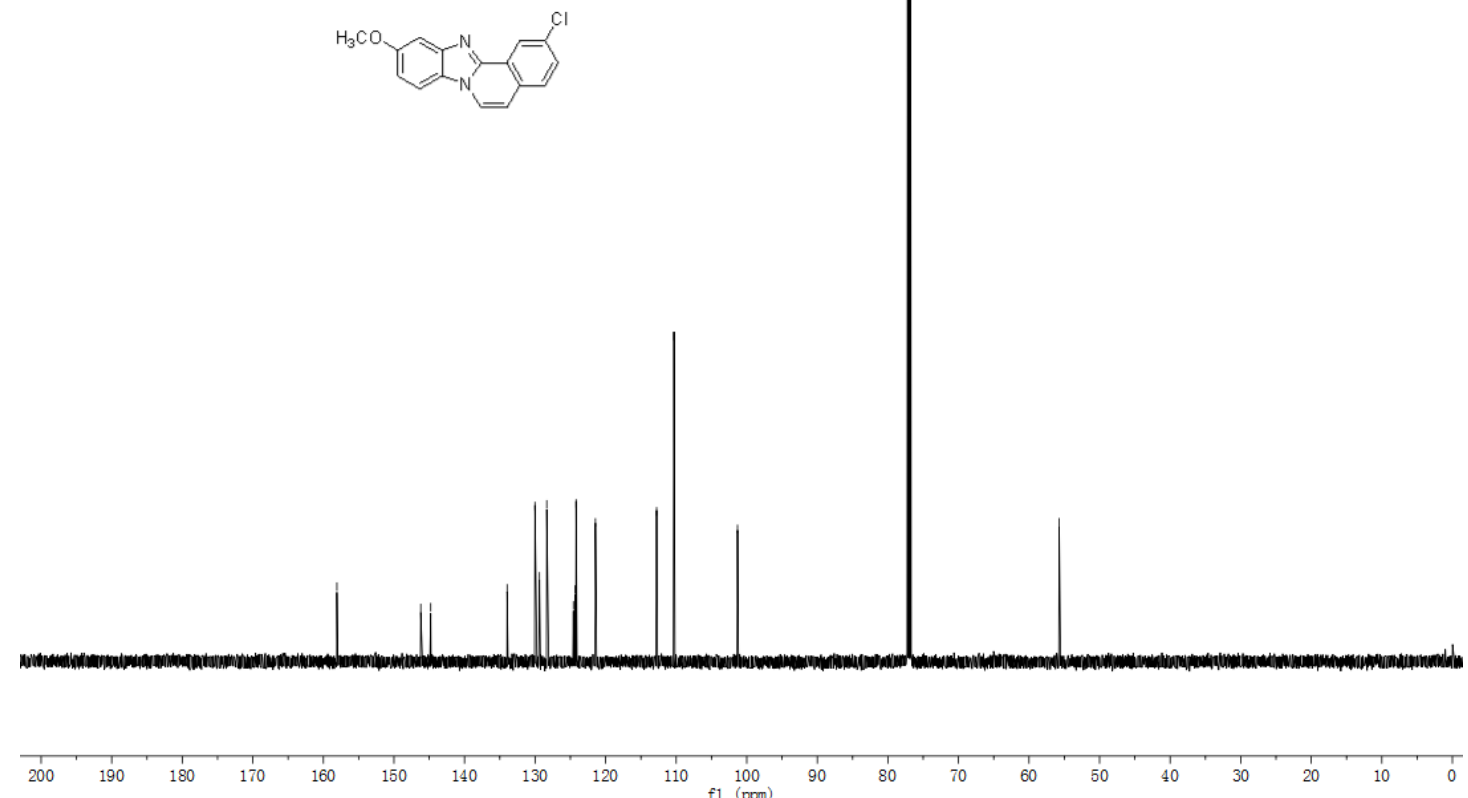

Fig S36. ${ }^{13} \mathrm{C} \mathrm{NMR}\left(150 \mathrm{MHz}, \mathrm{CDCl}_{3}\right)$ of 2-chloro-10-methoxybenzo[4,5]imidazo $[2,1-a]$ isoquinoline $(\mathbf{2 p})$ 


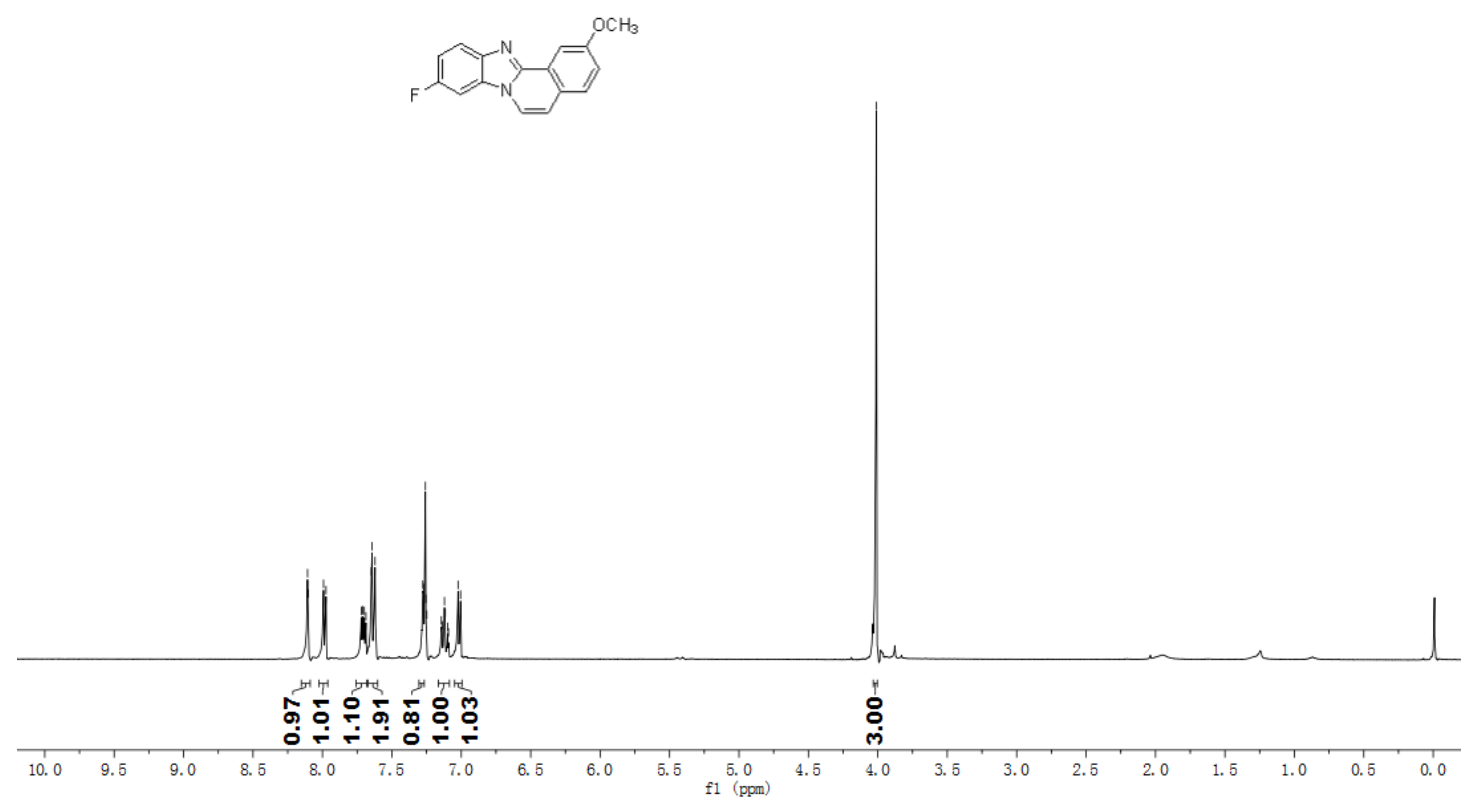

Fig S37. ${ }^{1} \mathrm{H}$ NMR (400 $\left.\mathrm{MHz}, \mathrm{CDCl}_{3}\right)$ of 9-fluoro-2-methoxybenzo[4,5]imidazo $[2,1-a]$ isoquinoline $(\mathbf{2 q})$
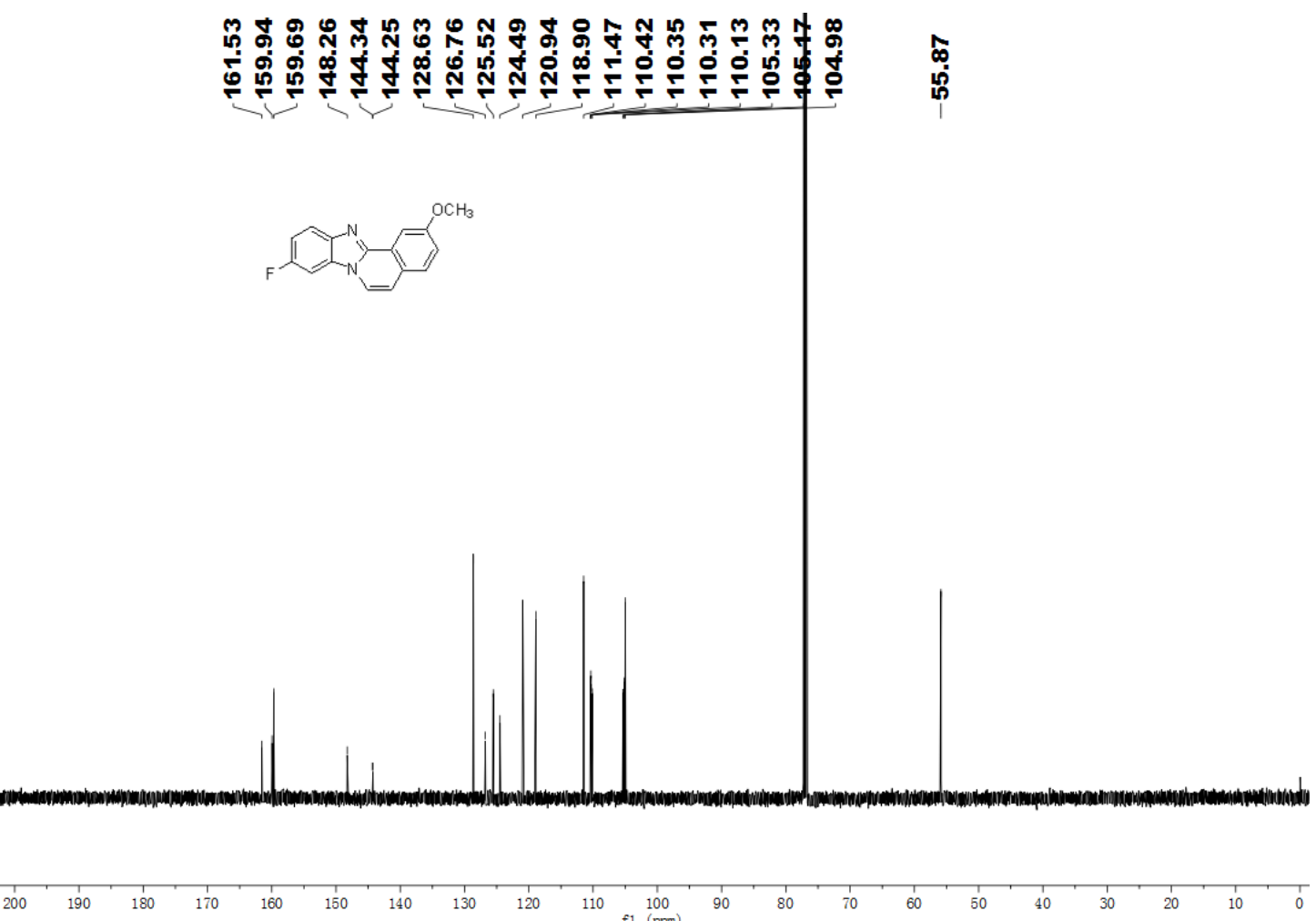

Fig S38. ${ }^{13} \mathrm{C}$ NMR $\left(150 \mathrm{MHz}, \mathrm{CDCl}_{3}\right)$ of 9-fluoro-2-methoxybenzo[4,5]imidazo [2,1- $a]$ isoquinoline (2q) 

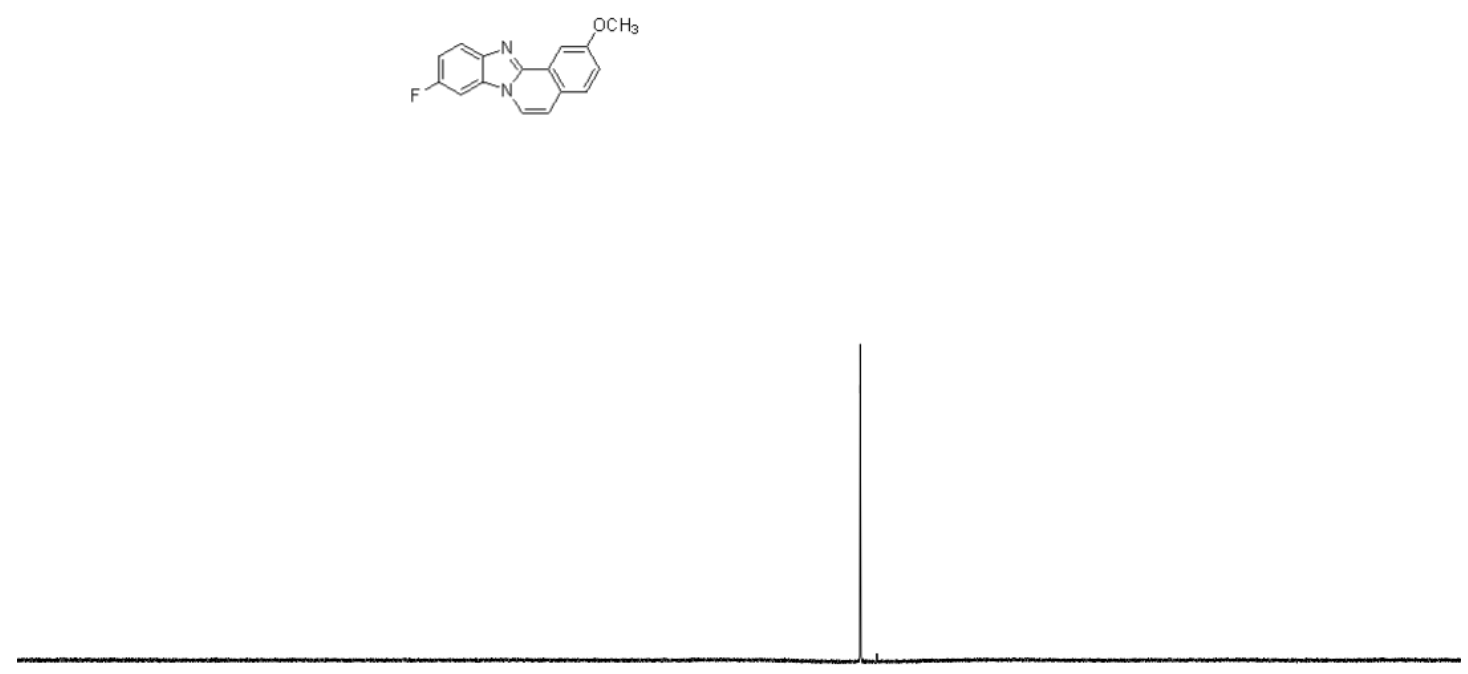

\begin{tabular}{lllllllllllllllllllllll}
\hline & 1 & -10 & -20 & -30 & -40 & -50 & -60 & -70 & -80 & -90 & -100 & -110 & -120 & -130 & -140 & -150 & -160 & -170 & -180 & -190 & -100
\end{tabular}

Fig S39. ${ }^{19} \mathrm{~F}$ NMR $\left(376 \mathrm{MHz}, \mathrm{CDCl}_{3}\right)$ of 10-fluoro-2-methoxybenzo[4,5]imidazo $[2,1-a]$ isoquinoline (2q)
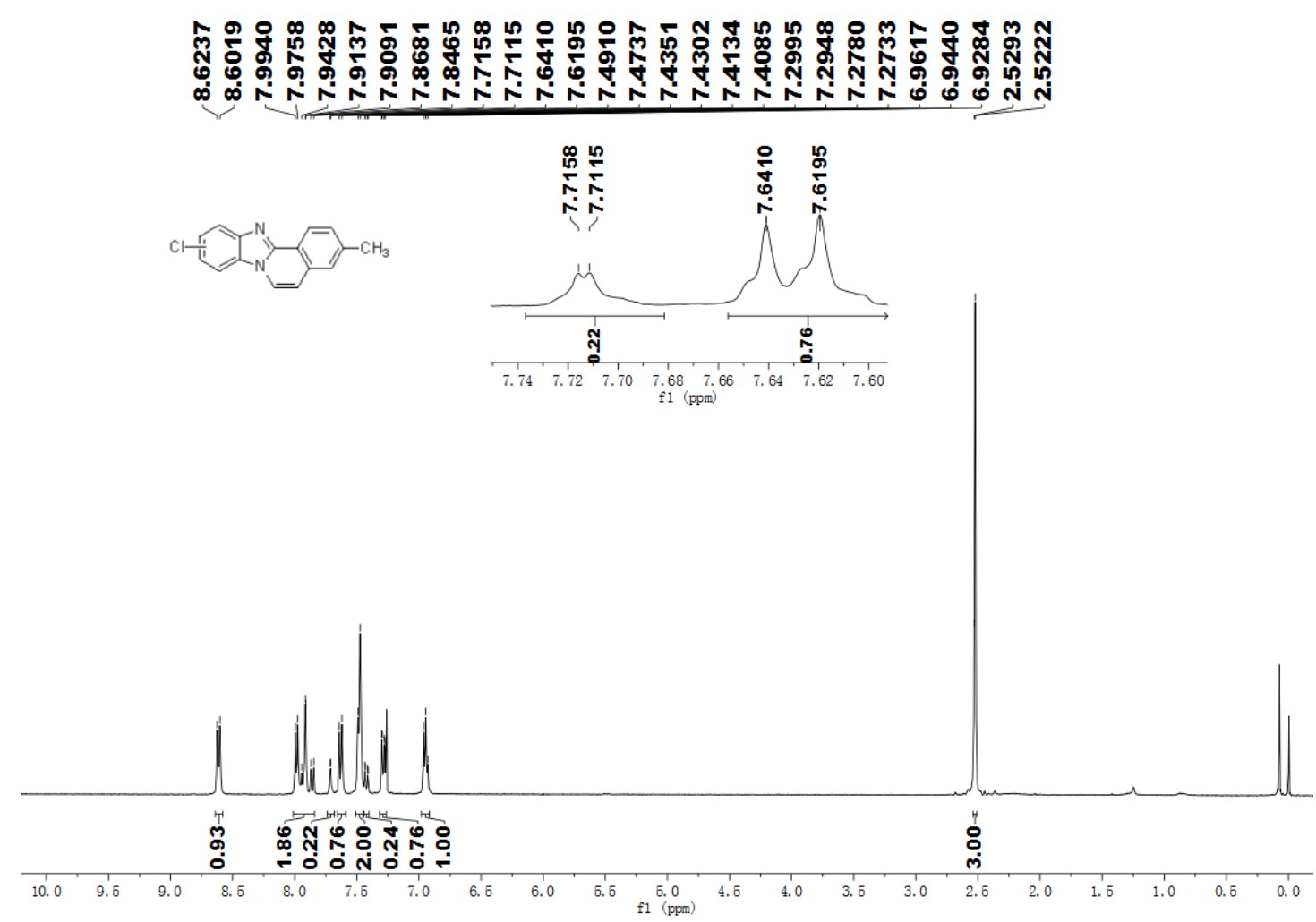

Fig S40. ${ }^{1} \mathrm{H}$ NMR (400 $\left.\mathrm{MHz}, \mathrm{CDCl}_{3}\right)$ of 10-chloro-3-methylbenzo[4,5]imidazo [2,1-a]isoquinoline and 9-chloro-3-methylbenzo[4,5]imidazo[2,1-a]isoquinoline (2r) 


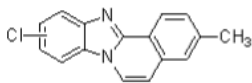

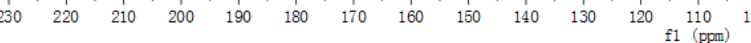

Fig S41. ${ }^{13} \mathrm{C}$ NMR $\left(150 \mathrm{MHz}, \mathrm{CDCl}_{3}\right)$ of 10-chloro-3-methylbenzo[4,5]imidazo [2,1-a]isoquinoline and 9-chloro-3-methylbenzo[4,5]imidazo[2,1-a] isoquinoline (2r)

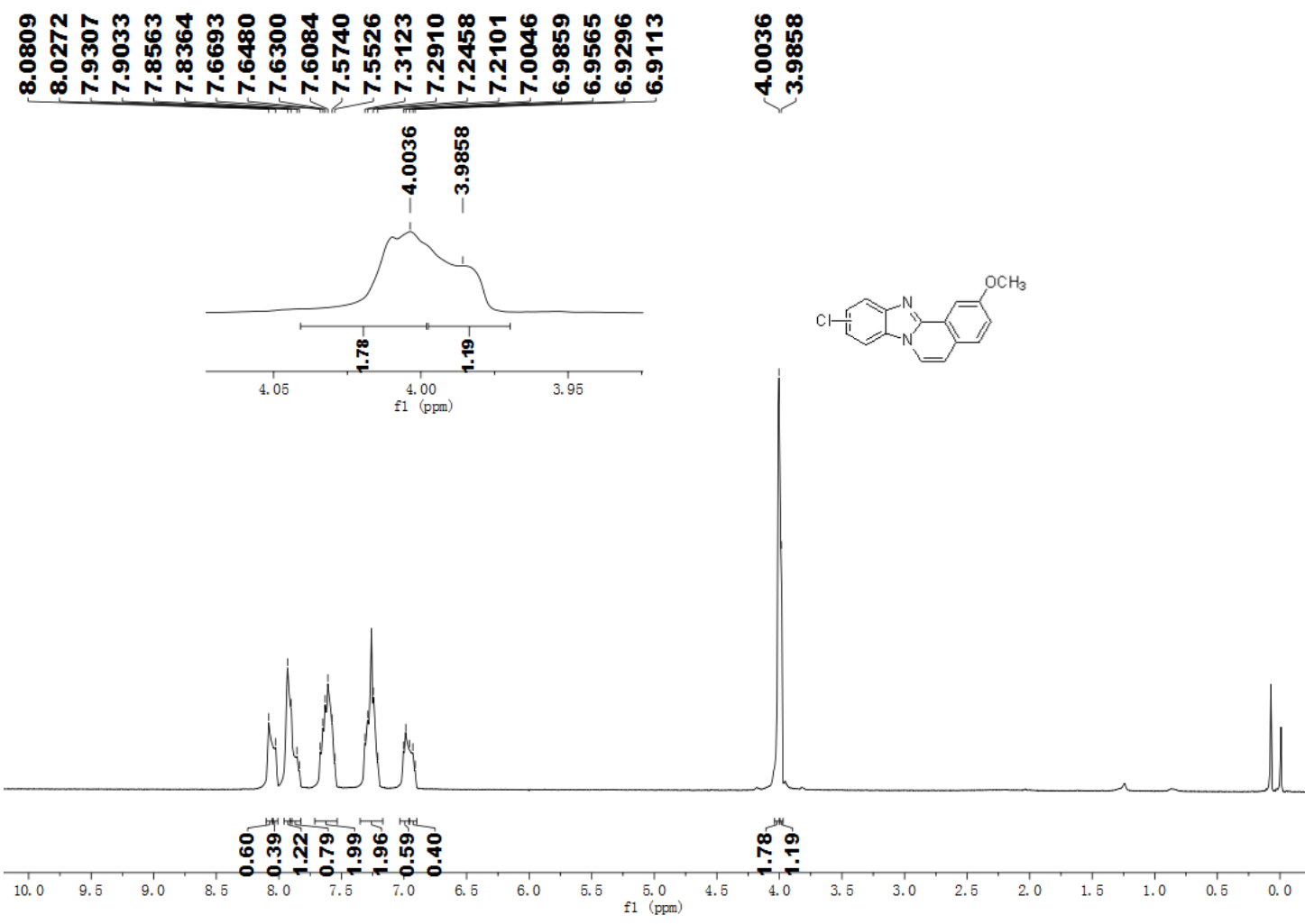

Fig S42. ${ }^{1} \mathrm{H}$ NMR (400 $\mathrm{MHz}, \mathrm{CDCl}_{3}$ ) of 10-chloro-2-methoxybenzo[4,5]imidazo $[2,1-a]$ isoquinoline and 9-chloro-2-methoxybenzo[4,5]imidazo[2,1-a]isoquinoline $(2 \mathrm{~s})$ 

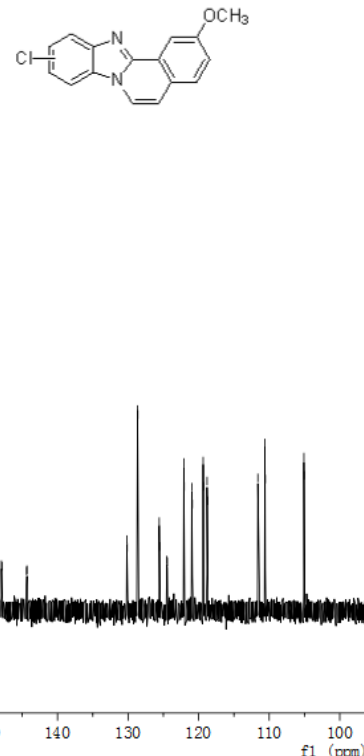

Fig S43. ${ }^{13} \mathrm{C}$ NMR $\left(150 \mathrm{MHz}, \mathrm{CDCl}_{3}\right)$ of 10-chloro-2-methoxybenzo[4,5]imidazo $[2,1-a]$ isoquinoline and 9-chloro-2-methoxybenzo[4,5]imidazo[2,1-a]isoquinoline $(2 \mathrm{~s})$
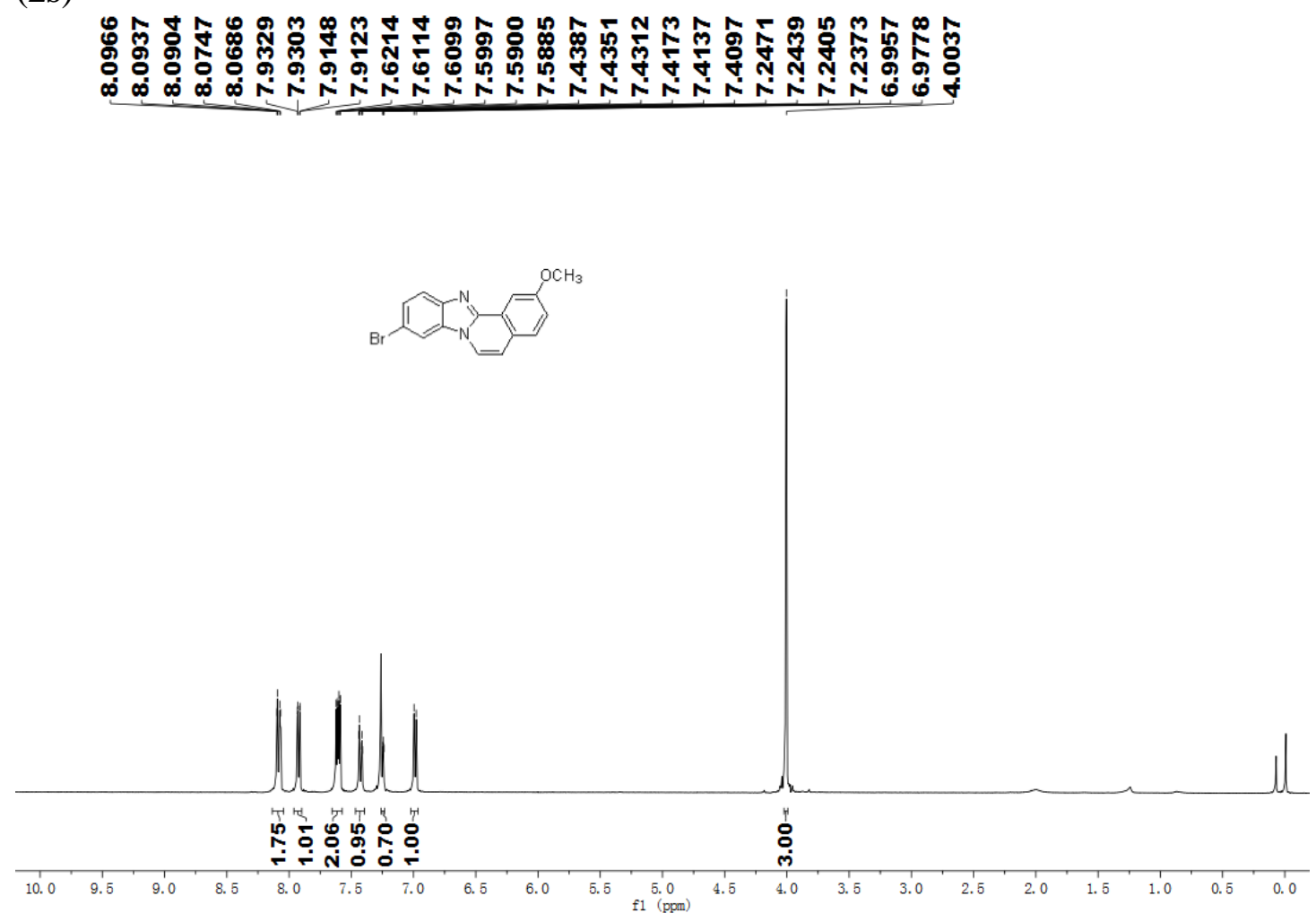

Fig S44. ${ }^{1} \mathrm{H}$ NMR $\left(400 \mathrm{MHz}, \mathrm{CDCl}_{3}\right)$ of 9-bromo-2-methoxybenzo[4,5]imidazo $[2,1-a]$ isoquinoline $(\mathbf{2 t})$ 


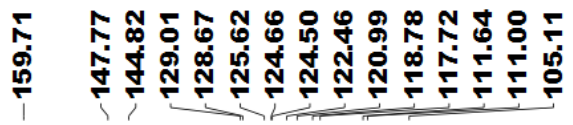

क્
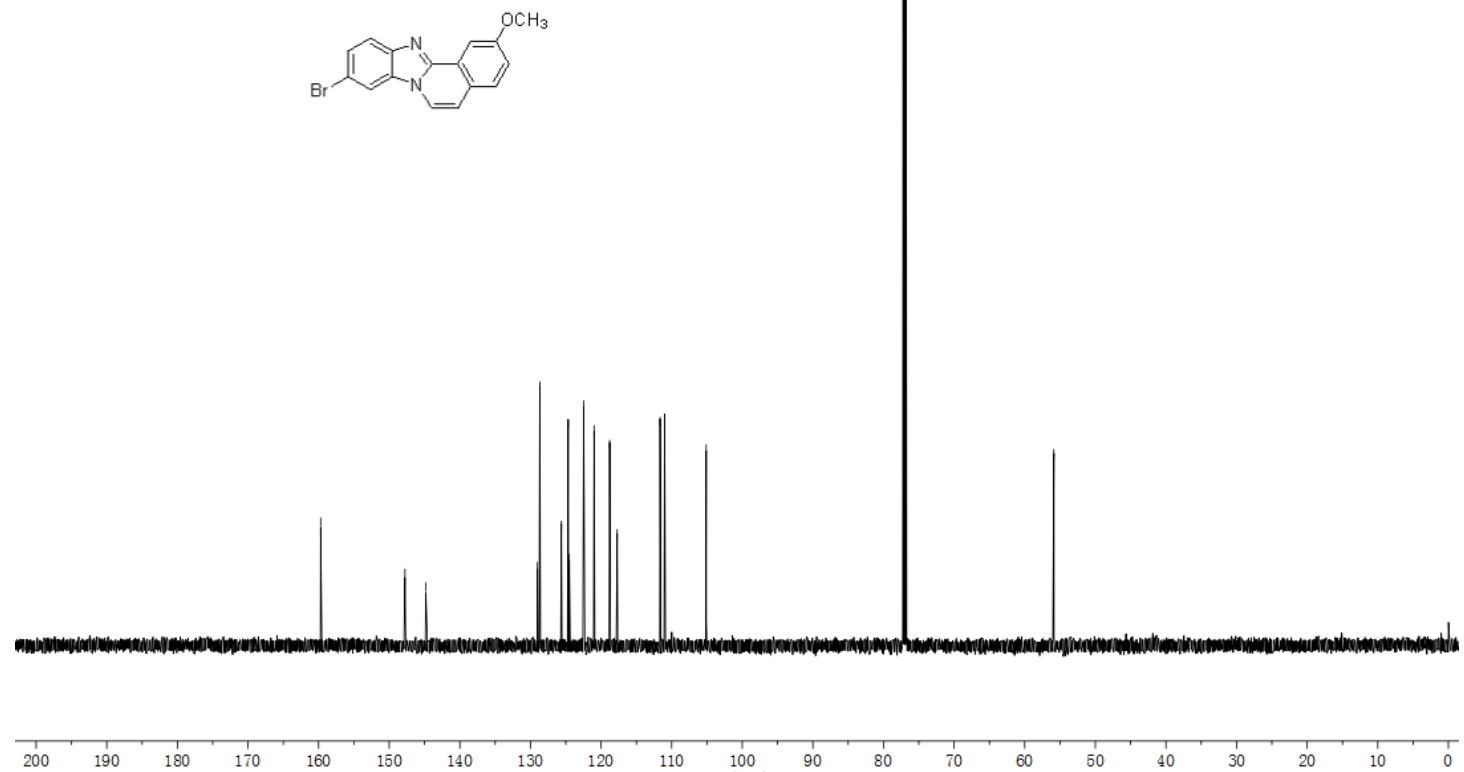

Fig S45. ${ }^{13} \mathrm{C}$ NMR $\left(150 \mathrm{MHz}, \mathrm{CDCl}_{3}\right)$ of 9-bromo-2-methoxybenzo[4,5]imidazo $[2,1-a]$ isoquinoline $(\mathbf{2 t})$

\section{ำ \\ 命}

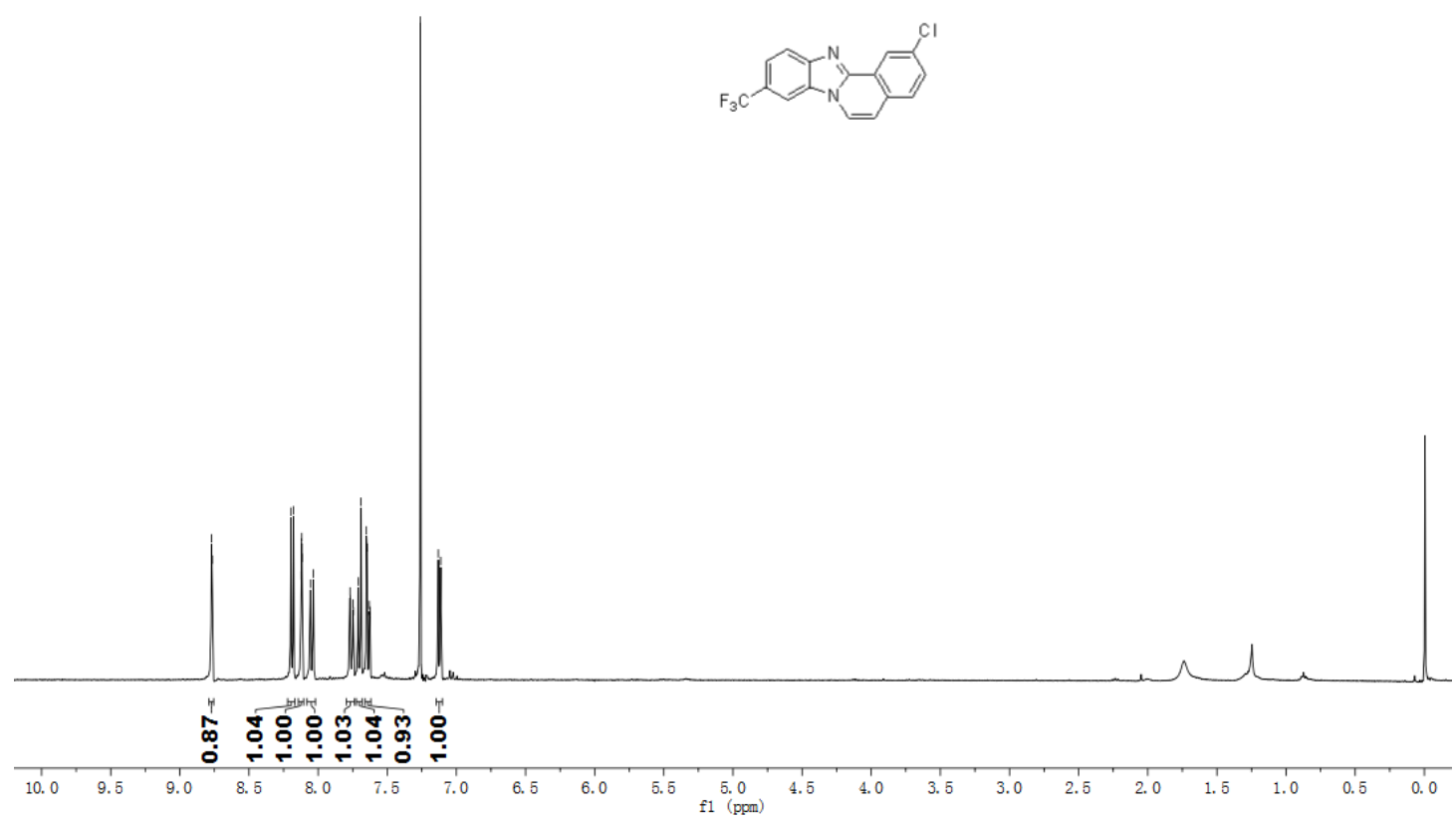

Fig S46. ${ }^{1} \mathrm{H}$ NMR (400 $\mathrm{MHz}, \mathrm{CDCl}_{3}$ ) of 2-chloro-9-(trifluoromethyl)benzo[4,5] imidazo[2,1-a]isoquinoline (2u) 

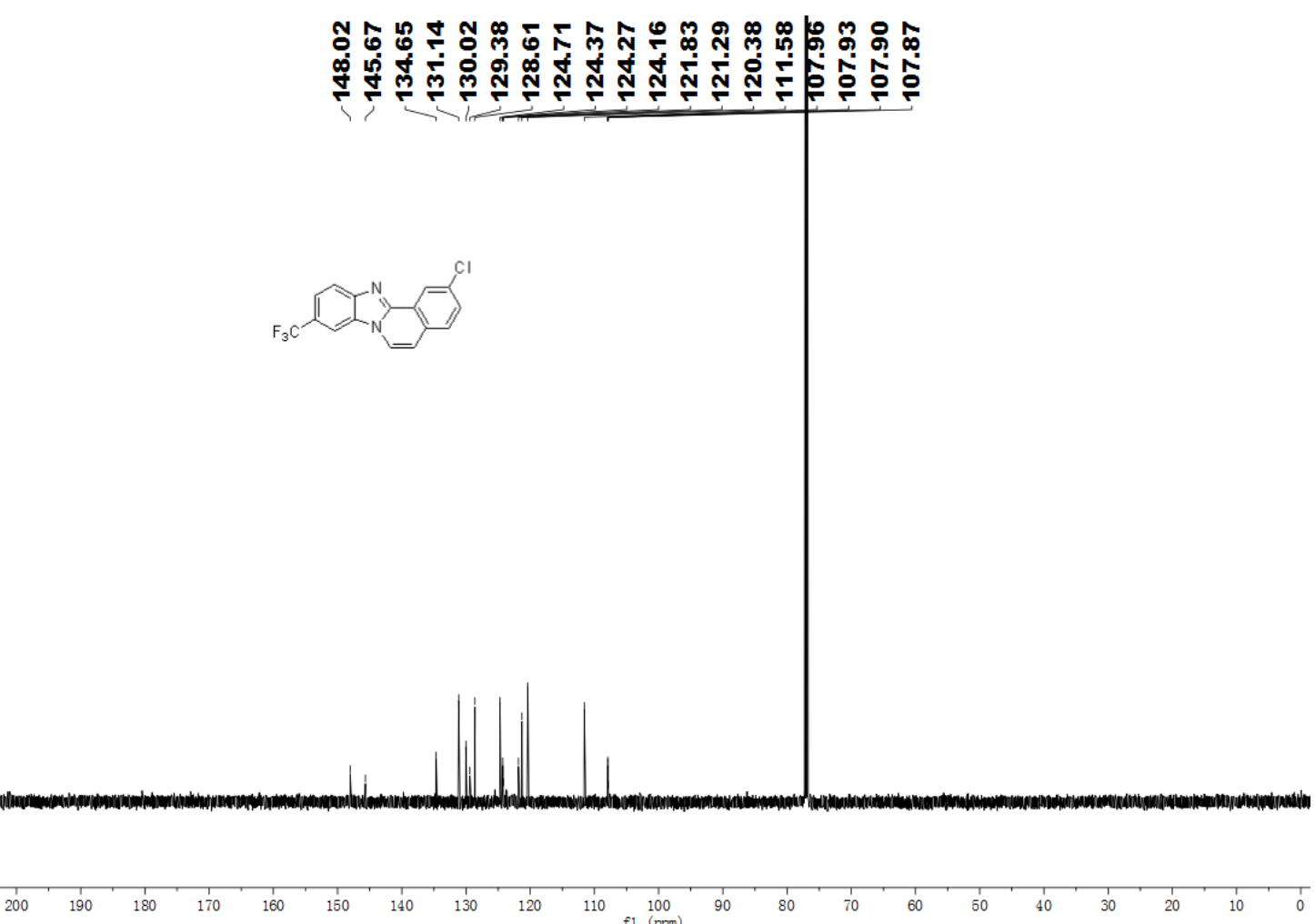

Fig S47. ${ }^{13} \mathrm{C} \mathrm{NMR}\left(150 \mathrm{MHz}, \mathrm{CDCl}_{3}\right)$ of 2-chloro-9-(trifluoromethyl)benzo[4,5] imidazo[2,1-a]isoquinoline $(\mathbf{2 u})$

ষ্ণ
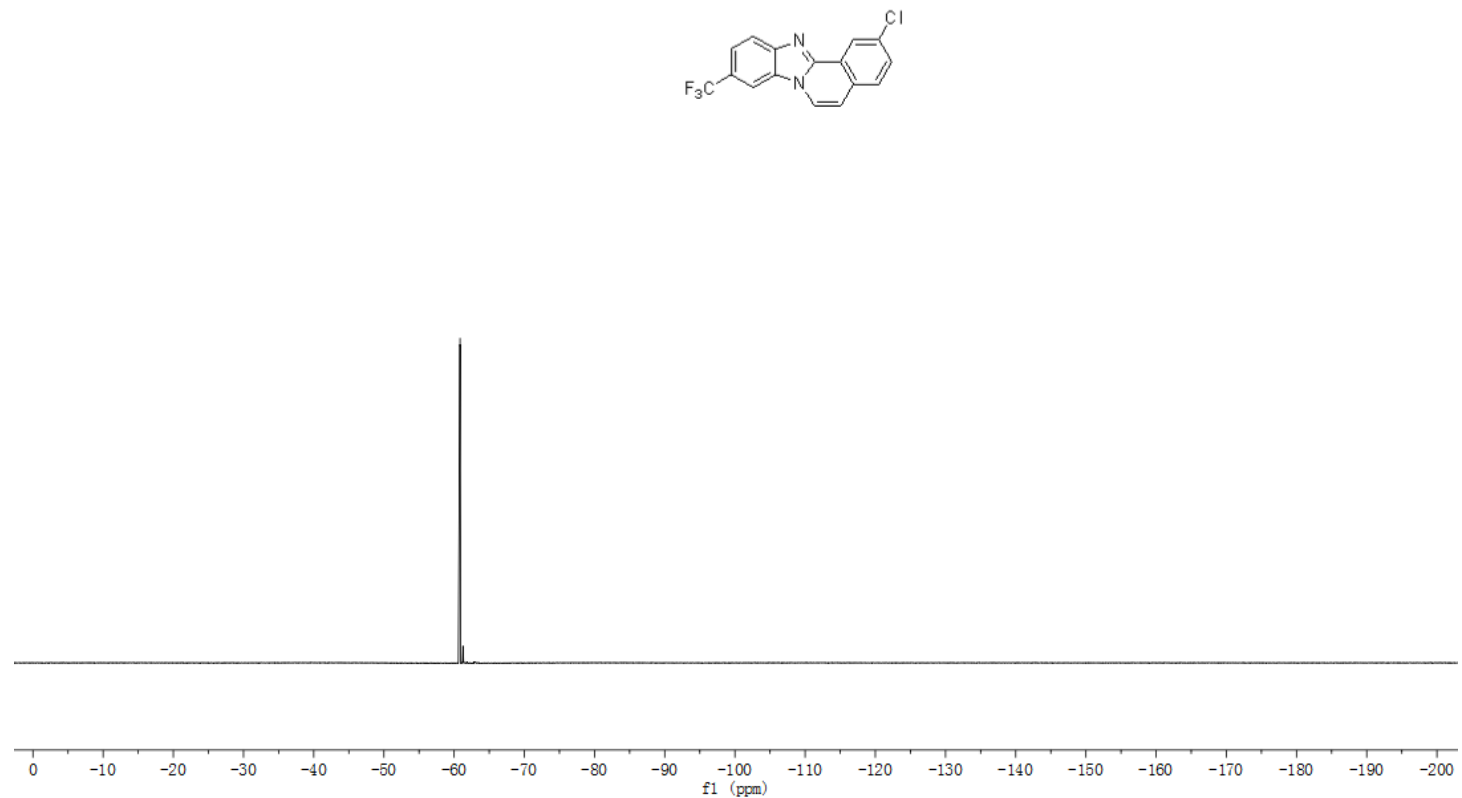

Fig S48. ${ }^{19} \mathrm{~F}$ NMR (376 $\mathrm{MHz}, \mathrm{CDCl}_{3}$ ) of 2-chloro-9-(trifluoromethyl)benzo[4,5] imidazo[2,1-a]isoquinoline (2u) 


\section{References}

[1] Lee, Y. S.; Cho, Y. H.; Lee, S. J.; Bin, J. K.; Yang, J. H.; Chae, G. S.; Cheon, C. H. Tetrahedron 2015, 71, 532-538.

[2] Mishra, M.; Twardy, D.; Ellstrom, C.; Wheeler, K. A.; Dembinski, R.; Torok, B. Green Chem. 2019, 21, 99-108.

[3] Koushik, G.; Yuji, N.; Masahiro, M. ACS Catal. 2019, 9, 11455-11460.

[4] Sun, M. M.; Wu, H. D.; Zheng, J. N.; Bao, W. L. Adv. Synth. Catal. 2012, 354, 835-838.

[5] Huang, J. H.; Zhu, R. R.; Wang, Y. T.; Liu, J. L.; Wang, Q. Synthesis 2017, 49, 1335-1341. 